\title{
Small Molecule Inhibitors of Dynamin I GTPase Activity: Development of Dimeric Tyrphostins
}

Timothy A. Hill, ${ }^{1}$ Luke R Odell, ${ }^{1}$ Jennifer K. Edwards, ${ }^{1}$ Mark E. Graham, ${ }^{2}$ Andrew B.

McGeachie, ${ }^{2}$ Jenny Rusak, ${ }^{2}$ Annie Quan, ${ }^{2}$ Ruben Abagyan, ${ }^{3}$ Janet L. Scott, ${ }^{4}$ Phillip J Robinson ${ }^{2 *}$ and Adam McCluskey ${ }^{1 *}$

${ }^{1}$ Medicinal Chemistry Group, Chemistry Building, School of Environmental and Life Sciences, The University of Newcastle, Callaghan, NSW 2308, Australia. E-mail:

Adam.McCluskey@newcastle.edu.au, Phone: +61 249 216486; Fax: +61 249215472

${ }^{2}$ Children’s Medical Research Institute, 214 Hawkesbury Road, Westmead NSW 2145, Australia. E-mail:phrobins@mail.usyd.edu.au

${ }^{3}$ Department of Molecular Biology, The Scripps Research Institute, 10550 North Torrey Pines Road, TCP-28, La Jolla, CA 92037, USA.

${ }^{4}$ Centre for Green Chemistry PO Box 23, Monash University, Victoria 3800, Australia

* Corresponding author

\section{Supplementary Data \#1}


Table. Micro analysis data

\begin{tabular}{|c|c|c|c|c|c|c|c|}
\hline Compound No & Mol Formula & C (Theor) & H (Theor) & N (Theor) & C (Meas) & H (Meas) & N (Meas) \\
\hline 10 & $\left(\mathrm{C}_{22} \mathrm{H}_{18} \mathrm{~N}_{4} \mathrm{O}_{8} \cdot 2 \mathrm{H}_{2} \mathrm{O}\right)$ & 52.59 & 4.41 & 11.15 & 52.21 & 4.28 & 11.17 \\
\hline 11 & $\left(\mathrm{C}_{24} \mathrm{H}_{22} \mathrm{~N}_{4} \mathrm{O}_{8} \cdot 0.5 \mathrm{H}_{2} \mathrm{O}\right)$ & 57.02 & 4.65 & 11.14 & 57.26 & 4.60 & 11.13 \\
\hline 24 & $\left(\mathrm{C}_{25} \mathrm{H}_{24} \mathrm{~N}_{4} \mathrm{O}_{8} \cdot 2 \mathrm{H}_{2} \mathrm{O}\right)$ & 55.25 & 4.98 & 10.67 & 55.14 & 5.18 & 10.29 \\
\hline 37 & $\left(\mathrm{C}_{26} \mathrm{H}_{26} \mathrm{~N}_{4} \mathrm{O}_{8 .} 1 \mathrm{H}_{2} \mathrm{O}\right)$ & 57.77 & 5.22 & 10.37 & 57.87 & 5.20 & 10.50 \\
\hline 50 & $\left(\mathrm{C}_{27} \mathrm{H}_{28} \mathrm{~N}_{4} \mathrm{O}_{8} .0 .5 \mathrm{H}_{2} \mathrm{O}\right)$ & 58.02 & 4.87 & 10.83 & 57.69 & 4.77 & 10.45 \\
\hline 62 & $\mathrm{C}_{26} \mathrm{H}_{26} \mathrm{~N}_{4} \mathrm{O}_{8} \cdot 2.5 \mathrm{H}_{2} \mathrm{O}$ & 55.02 & 5.51 & 9.87 & 54.94 & 5.28 & 10.26 \\
\hline 63 & $\mathrm{C}_{28} \mathrm{H}_{30} \mathrm{~N}_{4} \mathrm{O}_{8} \cdot 0.75 \mathrm{H}_{2} \mathrm{O}$ & 59.62 & 5.63 & 9.93 & 59.66 & 5.64 & 10.28 \\
\hline 82 & $\mathrm{C}_{23} \mathrm{H}_{20} \mathrm{~N}_{4} \mathrm{O}_{9} 2 \mathrm{H}_{2} \mathrm{O}$ & 55.20 & 4.83 & 11.19 & 55.33 & 5.19 & 11.25 \\
\hline 83 & $\mathrm{C}_{26} \mathrm{H}_{26} \mathrm{~N}_{4} \mathrm{O}_{7} \cdot 2 \mathrm{H}_{2} \mathrm{O}$ & 57.56 & 5.57 & 10.33 & 57.20 & 5.55 & 10.09 \\
\hline 84 & $\mathrm{C}_{30} \mathrm{H}_{26} \mathrm{~N}_{4} \mathrm{O}_{7} .0 .5 \mathrm{H}_{2} \mathrm{O}$ & 63.94 & 4.83 & 9.94 & 63.76 & 4.73 & 9.85 \\
\hline 85 & $\mathrm{C}_{23} \mathrm{H}_{20} \mathrm{~N}_{4} \mathrm{O}_{9} 0.4 \mathrm{H}_{2} \mathrm{O}$ & 58.82 & 4.96 & 11.43 & 58.87 & 4.96 & 11.61 \\
\hline 86 & $\mathrm{C}_{25} \mathrm{H}_{24} \mathrm{~N}_{4} \mathrm{O}_{6} \cdot 2 \cdot 0 \mathrm{H}_{2} \mathrm{O}$ & 57.83 & 5.26 & 11.24 & 57.86 & 5.09 & 11.24 \\
\hline 87 & $\mathrm{C}_{24} \mathrm{H}_{22} \mathrm{~N}_{4} \mathrm{O}_{6} \cdot 2 \cdot 5 \mathrm{H}_{2} \mathrm{O}$ & 57.58 & 5.60 & 10.74 & 57.70 & 5.53 & 10.50 \\
\hline 90 & $\mathrm{C}_{22} \mathrm{H}_{16} \mathrm{~N}_{2} \mathrm{O}_{8} \cdot 0.25 \mathrm{H}_{2} \mathrm{O}$ & 59.93 & 3.77 & 6.35 & 59.80 & 4.09 & 6.40 \\
\hline 91 & $\mathrm{C}_{23} \mathrm{H}_{18} \mathrm{~N}_{2} \mathrm{O}_{8} \cdot 0.5 \mathrm{H}_{2} \mathrm{O}$ & 60.13 & 4.17 & 6.10 & 60.00 & 4.47 & 6.23 \\
\hline 92 & $\mathrm{C}_{24} \mathrm{H}_{20} \mathrm{~N}_{2} \mathrm{O}_{8} \cdot 0.25 \mathrm{H}_{2} \mathrm{O}$ & 61.47 & 4.41 & 5.97 & 61.65 & 4.66 & 6.16 \\
\hline 93 & $\mathrm{C}_{26} \mathrm{H}_{24} \mathrm{~N}_{2} \mathrm{O}_{8} \cdot 1 \mathrm{H}_{2} \mathrm{O}$ & 61.17 & 5.13 & 5.49 & 61.34 & 4.85 & 5.89 \\
\hline 98 & $\mathrm{C}_{20} \mathrm{H}_{20} \mathrm{~N}_{2} \mathrm{O}_{6} \cdot 0.5 \mathrm{H}_{2} \mathrm{O}$ & 61.06 & 5.38 & 7.12 & 61.32 & 5.44 & 7.29 \\
\hline 99 & $\mathrm{C}_{21} \mathrm{H}_{22} \mathrm{~N}_{2} \mathrm{O}_{6} \cdot 1 \mathrm{H}_{2} \mathrm{O}$ & 60.57 & 5.81 & 6.73 & 60.46 & 5.39 & 6.38 \\
\hline 100 & $\mathrm{C}_{22} \mathrm{H}_{24} \mathrm{~N}_{2} \mathrm{O}_{6} \cdot 1 \cdot 25 \mathrm{H}_{2} \mathrm{O}$ & 60.75 & 6.14 & 6.44 & 60.24 & 5.85 & 6.44 \\
\hline 101 & $\mathrm{C}_{24} \mathrm{H}_{28} \mathrm{~N}_{2} \mathrm{O}_{6} \cdot 2.5 \mathrm{H}_{2} \mathrm{O}$ & 59.37 & 6.85 & 5.77 & 59.64 & 6.57 & 5.34 \\
\hline 102 & $\mathrm{C}_{30} \mathrm{H}_{40} \mathrm{~N}_{2} \mathrm{O}_{6} \cdot 0.75 \mathrm{H}_{2} \mathrm{O}$ & 66.95 & 7.77 & 5.21 & 66.99 & 7.43 & 4.85 \\
\hline
\end{tabular}




\section{General Experimental}

General: All starting materials were purchased from Aldrich Chemical Company and Lancaster Synthesis. Solvents were bulk, and distilled from glass prior to use. ${ }^{1} \mathrm{H}$ and ${ }^{13} \mathrm{C}$ spectra were recorded on a Bruker Advance AMX $300 \mathrm{MHz}$ spectrometer at 300.13 and $75.48 \mathrm{MHz}$ respectively. Chemical shifts are relative to TMS as internal standard. All compounds returned satisfactory analyses.
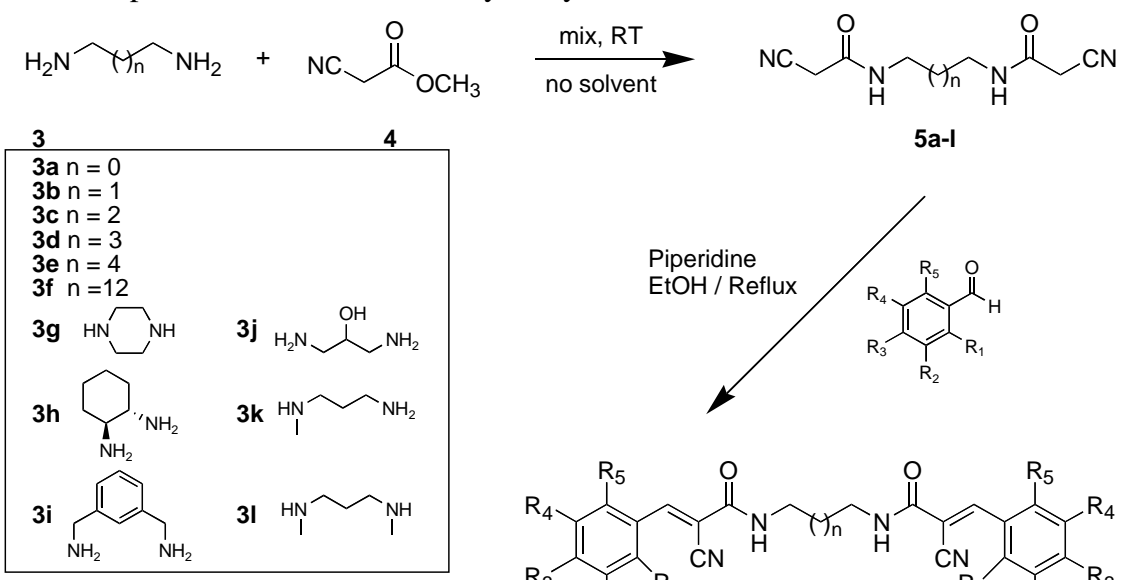

5a-I
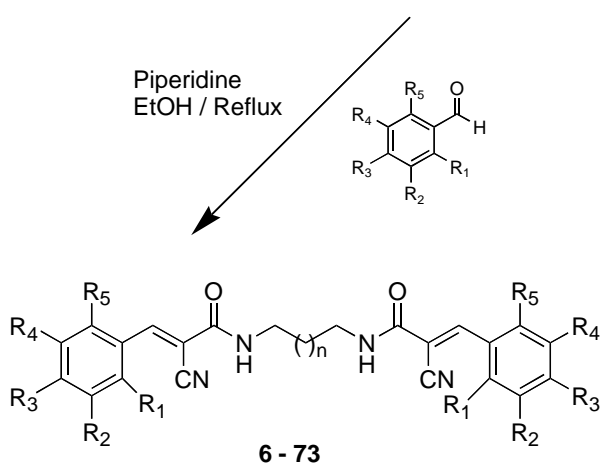

Scheme 1. Synthesis of a dimeric analogue of 2 library. Details of substituents $R_{1}-R_{5}$ and the alkane spacer $n$ are given in the Table.

\begin{tabular}{|c|c|c|c|c|c|c|}
\hline Compound & $\mathbf{R}^{1}$ & $\mathbf{R}^{2}$ & $\mathbf{R}^{3}$ & $\mathbf{R}^{4}$ & $\mathbf{R}^{5}$ & $\mathbf{n}$ \\
\hline 6 & $\mathrm{H}$ & $\overline{\mathrm{H}}$ & $\mathrm{H}$ & $\mathrm{H}$ & $\mathrm{H}$ & 0 \\
\hline 7 & $\mathrm{H}$ & $\mathrm{OH}$ & $\mathrm{H}$ & $\mathrm{H}$ & $\mathrm{H}$ & 0 \\
\hline 8 & $\mathrm{H}$ & $\mathrm{H}$ & $\mathrm{OH}$ & $\mathrm{H}$ & $\mathrm{H}$ & 0 \\
\hline 12 & $\mathrm{Cl}$ & $\mathrm{H}$ & $\mathrm{H}$ & $\mathrm{H}$ & $\mathrm{H}$ & 0 \\
\hline 13 & $\mathrm{H}$ & $\mathrm{H}$ & $\mathrm{H}$ & $\mathrm{Cl}$ & $\mathrm{H}$ & 0 \\
\hline 14 & $\mathrm{H}$ & $\mathrm{OMe}$ & $\mathrm{H}$ & $\mathrm{H}$ & $\mathrm{H}$ & 0 \\
\hline 15 & $\mathrm{H}$ & $\mathrm{H}$ & OMe & $\mathrm{H}$ & $\mathrm{H}$ & 0 \\
\hline 16 & $\mathrm{H}$ & $\mathrm{H}$ & OMe & $\mathrm{OMe}$ & $\mathrm{H}$ & 0 \\
\hline 17 & $\mathrm{H}$ & $\mathrm{H}$ & OMe & $\mathrm{OH}$ & $\mathrm{H}$ & 0 \\
\hline 18 & $\mathrm{H}$ & $\mathrm{H}$ & $\mathrm{COOH}$ & $\mathrm{H}$ & $\mathrm{H}$ & 0 \\
\hline 19 & $\mathrm{H}$ & $\mathrm{H}$ & $\mathrm{H}$ & $\mathrm{H}$ & $\mathrm{H}$ & 1 \\
\hline 20 & $\mathrm{H}$ & $\mathrm{OH}$ & $\mathrm{H}$ & $\mathrm{H}$ & $\mathrm{H}$ & 1 \\
\hline 21 & $\mathrm{H}$ & $\mathrm{H}$ & $\mathrm{OH}$ & $\mathrm{H}$ & $\mathrm{H}$ & 1 \\
\hline 25 & $\mathrm{Cl}$ & $\mathrm{H}$ & $\mathrm{H}$ & $\mathrm{H}$ & $\mathrm{H}$ & 1 \\
\hline 26 & $\mathrm{H}$ & $\mathrm{H}$ & $\mathrm{Cl}$ & $\mathrm{H}$ & $\mathrm{H}$ & 1 \\
\hline 27 & $\mathrm{H}$ & $\mathrm{OMe}$ & $\mathrm{H}$ & $\mathrm{H}$ & $\mathrm{H}$ & 1 \\
\hline 28 & $\mathrm{H}$ & $\mathrm{H}$ & OMe & $\mathrm{H}$ & $\mathrm{H}$ & 1 \\
\hline 29 & $\mathrm{H}$ & $\mathrm{H}$ & OMe & OMe & $\mathrm{H}$ & 1 \\
\hline 30 & $\mathrm{H}$ & $\mathrm{H}$ & OMe & $\mathrm{OH}$ & $\mathrm{H}$ & 1 \\
\hline 31 & $\mathrm{H}$ & $\mathrm{H}$ & $\mathrm{COOH}$ & $\mathrm{H}$ & $\mathrm{H}$ & 1 \\
\hline 32 & $\mathrm{H}$ & $\mathrm{H}$ & $\mathrm{H}$ & $\mathrm{H}$ & $\mathrm{H}$ & 2 \\
\hline 33 & $\mathrm{H}$ & $\mathrm{OH}$ & $\mathrm{H}$ & $\mathrm{H}$ & $\mathrm{H}$ & 2 \\
\hline 34 & $\mathrm{H}$ & $\mathrm{H}$ & $\mathrm{OH}$ & $\mathrm{H}$ & $\mathrm{H}$ & 2 \\
\hline
\end{tabular}




$\begin{array}{lllllll}\mathbf{3 8} & \mathrm{Cl} & \mathrm{H} & \mathrm{H} & \mathrm{H} & \mathrm{H} & 2 \\ \mathbf{3 9} & \mathrm{H} & \mathrm{H} & \mathrm{H} & \mathrm{Cl} & \mathrm{H} & 2 \\ \mathbf{4 0} & \mathrm{H} & \mathrm{OMe} & \mathrm{H} & \mathrm{H} & \mathrm{H} & 2 \\ \mathbf{4 1} & \mathrm{H} & \mathrm{H} & \mathrm{OMe} & \mathrm{H} & \mathrm{H} & 2 \\ \mathbf{4 2} & \mathrm{H} & \mathrm{H} & \mathrm{OMe} & \mathrm{OMe} & \mathrm{H} & 2 \\ \mathbf{4 3} & \mathrm{H} & \mathrm{H} & \mathrm{OMe} & \mathrm{OH} & \mathrm{H} & 2 \\ \mathbf{4 4} & \mathrm{H} & \mathrm{H} & \mathrm{COOH} & \mathrm{H} & \mathrm{H} & 2 \\ \mathbf{4 5} & \mathrm{H} & \mathrm{H} & \mathrm{H} & \mathrm{H} & \mathrm{H} & 3 \\ \mathbf{4 6} & \mathrm{H} & \mathrm{OH} & \mathrm{H} & \mathrm{H} & \mathrm{H} & 3 \\ \mathbf{4 7} & \mathrm{H} & \mathrm{H} & \mathrm{OH} & \mathrm{H} & \mathrm{H} & 3 \\ \mathbf{5 1} & \mathrm{Cl} & \mathrm{H} & \mathrm{H} & \mathrm{H} & \mathrm{H} & 3 \\ \mathbf{5 2} & \mathrm{H} & \mathrm{H} & \mathrm{H} & \mathrm{Cl} & \mathrm{H} & 3 \\ \mathbf{5 3} & \mathrm{H} & \mathrm{OMe} & \mathrm{H} & \mathrm{H} & \mathrm{H} & 3 \\ \mathbf{5 4} & \mathrm{H} & \mathrm{H} & \mathrm{OMe} & \mathrm{H} & \mathrm{H} & 3 \\ \mathbf{5 5} & \mathrm{H} & \mathrm{H} & \mathrm{OMe} & \mathrm{OMe} & \mathrm{H} & 3 \\ \mathbf{5 6} & \mathrm{H} & \mathrm{H} & \mathrm{OMe} & \mathrm{OH} & \mathrm{H} & 3 \\ \mathbf{5 7} & \mathrm{H} & \mathrm{H} & \mathrm{COOH} & \mathrm{H} & \mathrm{H} & 3 \\ \mathbf{5 8} & \mathrm{H} & \mathrm{H} & \mathrm{H} & \mathrm{H} & \mathrm{H} & 4 \\ \mathbf{5 9} & \mathrm{H} & \mathrm{OH} & \mathrm{H} & \mathrm{H} & \mathrm{H} & 4 \\ \mathbf{6 0} & \mathrm{H} & \mathrm{H} & \mathrm{OH} & \mathrm{H} & \mathrm{H} & 4 \\ \mathbf{6 4} & \mathrm{Cl} & \mathrm{H} & \mathrm{H} & \mathrm{H} & \mathrm{H} & 4 \\ \mathbf{6 5} & \mathrm{H} & \mathrm{H} & \mathrm{H} & \mathrm{Cl} & \mathrm{H} & 4 \\ \mathbf{6 6} & \mathrm{H} & \mathrm{OMe} & \mathrm{H} & \mathrm{H} & \mathrm{H} & 4 \\ \mathbf{6 7} & \mathrm{H} & \mathrm{H} & \mathrm{OMe} & \mathrm{H} & \mathrm{H} & 4 \\ \mathbf{6 8} & \mathrm{H} & \mathrm{H} & \mathrm{OMe} & \mathrm{OMe} & \mathrm{H} & 4 \\ \mathbf{6 9} & \mathrm{H} & \mathrm{H} & \mathrm{OMe} & \mathrm{OH} & \mathrm{H} & 4 \\ \mathbf{7 0} & \mathrm{H} & \mathrm{H} & \mathrm{COOH} & \mathrm{H} & \mathrm{H} & 4 \\ \mathbf{7 1} & \mathrm{H} & \mathrm{OH} & \mathrm{OH} & \mathrm{H} & \mathrm{H} & 12 \\ \mathbf{7 2} & \mathrm{H} & \mathrm{OH} & \mathrm{OH} & \mathrm{OH} & \mathrm{H} & 12 \\ \mathbf{7 3} & \mathrm{H} & \mathrm{OMe} & \mathrm{OH} & \mathrm{OH} & \mathrm{H} & 12\end{array}$

Synthetic methods

Synthesis of active analogues

DATA SHOWN IN TABLE 1 OF MANUSCRIPT

2-Cyano-N-[3-(2-cyanoacetylamino)ethyl] acetamide (5a).

Ethylenediamine (3a) $(1.5 \mathrm{~g}, 25 \mathrm{mmol})$ and methylcyanoacetate (4) (5 g, $50 \mathrm{mmol})$ were stirred at room temperature for 2 hours. The resulting white solid was then mixed with $10 \mathrm{~mL}$ ethanol and collected by filtration. Recrystallization from ethanol afforded a white solid in $81 \%$, 6.3g, mp $182^{\circ} \mathrm{C}$ (Lit. 183). ${ }^{[1]}$

${ }^{1} \mathrm{H}$ NMR (DMSO): 8.25 (2H, t, $\left.J=5.5 \mathrm{~Hz}\right), 3.56$ (4H, s), 3.13 (4H, br s).

${ }^{13}$ C NMR (DMSO): 162.3, 115.9, 38.4, 25.3.

2-Cyano- $N$-\{3-[2-cyano-3-(3,4-dihydroxyphenyl)acryloylamino]ethyl\}-3-(3,4-dihydroxyphenyl)acrylamide (9).

2-Cyano-N-[3-(2-cyanoacetylamino)ethyl]acetamide (5a) $(0.3 \mathrm{~g}, 1.5 \mathrm{mmol})$, 3,4-dihydroxybenzaldehyde $(0.42 \mathrm{~g}, 3$ $\mathrm{mmol}), 3$ drops piperidine and ethanol $(10 \mathrm{~mL})$ were refluxed together for two hours. Cooling, filtering and washing the solid with cold ether $(100 \mathrm{~mL})$ afforded a yellow-green solid $(9), 81 \%, 0.54 \mathrm{~g}, \mathrm{mp} 290^{\circ} \mathrm{C}\left(\mathrm{Lit} 295^{\circ} \mathrm{C}\right) .^{[1]}$

${ }^{1} \mathrm{H}$ NMR (DMSO): 8.32 (2H, t, $\left.J=5.5 \mathrm{~Hz}\right), 7.92(2 \mathrm{H}, \mathrm{s}), 7.53(2 \mathrm{H}, \mathrm{d}, J=2.1 \mathrm{~Hz}), 7.25$ (2H, dd, $J=8.2,2.1 \mathrm{~Hz}$ ), $6.85(2 \mathrm{H}, \mathrm{d}, J=2.1 \mathrm{~Hz}), 3.45$ (4H, br s).

${ }^{13}$ C NMR (DMSO): 162.5, 151.6, 161.6, 146.2, 125.8, 123.5, 117.7, 116.5, 116.3, 100.8, 39.6.

2-Cyano-N-[3-(2-cyanoacetylamino)propyl]acetamide (5b).

Synthesized as per $\mathbf{5 a}$ from 1,3-diaminopropane (3b) and methylcyanoacetate (4). Afforded a white solid, 81\%, mp $146^{\circ} \mathrm{C}\left(\right.$ Lit $\left.148^{\circ} \mathrm{C}\right) .{ }^{[1]}$

${ }^{1} \mathrm{H}$ NMR (DMSO): 8.21 (2H, t, $J=5.5 \mathrm{~Hz}$ ), 3.59 (4H, s), 3.07 (4H, q, $J=6.7 \mathrm{~Hz}$ ), 1.53 (2H, quin, $J=6.7 \mathrm{~Hz}$ ).

${ }^{13}$ C NMR (DMSO): 162.5, 116.6, 39.3, 28.9, 25.7. 
2-Cyano- $N$-\{3-[2-cyano-3-(3,4-dihydroxyphenyl)acryloylamino]propyl\}-3-(3,4-dihydroxyphenyl)acrylamide (22).

Synthesized as per $\mathbf{9}$ from 2-cyano- $N$-[3-(2-cyanoacetylamino)propyl]acetamide (5b) and 3,4dihyroxybenzaldehyde. Afforded a yellow-green solid, 85\%, mp $274^{\circ} \mathrm{C}$ (Lit $\left.277^{\circ} \mathrm{C}\right) .^{[1]}$

${ }^{1} \mathrm{H}$ NMR (DMSO): 8.24 (2H, t, $\left.J=5.5 \mathrm{~Hz}\right), 7.92(\mathrm{~s}, 2 \mathrm{H}), 7.52(2 \mathrm{H}, \mathrm{d}, J=2.1 \mathrm{~Hz}), 7.26(2 \mathrm{H}, \mathrm{dd}, J=8.2,2.1 \mathrm{~Hz})$, $6.85(2 \mathrm{H}, \mathrm{d}, J=8.2 \mathrm{~Hz}), 3.23(4 \mathrm{H}, \mathrm{q}, J=6 \mathrm{~Hz}), 1.70(2 \mathrm{H}$, quin, $J=6.7 \mathrm{~Hz})$.

${ }^{13}$ C NMR (DMSO): 161.5, 150.6, 150.5, 125.1, 123.2, 117.1, 116.0, 115.8, 100.5, 37.3, 28.8. (23).

2-Cyano- $N$-\{3-[2-cyano-3-(3,4,5-trihydroxyphenyl)acryloylamino]propyl\}-3-(3,4,5-trihydroxyphenyl)acrylamide

Synthesized as per $\mathbf{9}$ from 2-cyano- $N$-[3-(2-cyanoacetylamino)propyl]acetamide (5b) and 3,4,5trihyroxybenzaldehyde. Afforded an orange solid, $70 \%, \mathrm{Mp}>300^{\circ} \mathrm{C}$ (Lit $\left.>300^{\circ} \mathrm{C}\right) .^{[1]}$

${ }^{1} \mathrm{H}$ NMR (DMSO): 8.18 (2H, t, $\left.J=5.5 \mathrm{~Hz}\right), 7.78(2 \mathrm{H}, \mathrm{s}), 6.99(4 \mathrm{H}, \mathrm{s}), 3.21(4 \mathrm{H}, \mathrm{q}, J=6.8 \mathrm{~Hz}), 1.68(2 \mathrm{H}$, quin, $J=$ $6.8 \mathrm{~Hz})$

${ }^{13}$ C NMR (DMSO): 161.8, 150.7, 145.9, 140.3, 121.2, 117.3, 109.9, 99.5, 38.2, 28.9.

2-Cyano- $N$-[3-(2-cyanoacetylamino)butyl]acetamide (5c).

Synthesized as per $5 a$ from 1,4-diaminobutane (3c) and methylcyanoacetate (4). Afforded a white solid, 78\%, mp $145^{\circ} \mathrm{C}$ (Lit $\left.145^{\circ} \mathrm{C}\right) .^{[1]}$

${ }^{1} \mathrm{H}$ NMR (DMSO): 8.15 (2H, t, $\left.J=5.5 \mathrm{~Hz}\right), 3.56$ (4H, s), 3.05 (4H, br s), 1.38 (4H, br s)

${ }^{13} \mathrm{C}$ NMR (DMSO): 161.8, 116.1, 38.6, 26.1, 25.2 .

2-Cyano- $N$-\{3-[2-cyano-3-(3,4-dihydroxyphenyl)acryloylamino]butyl\}-3-(3,4-dihydroxyphenyl)acrylamide (35).

Synthesized as per $\mathbf{9}$ from 2-cyano- $N$-[3-(2-cyanoacetylamino)butyl]acetamide (5c) and 3,4dihydroxybenzaldehyde. Afforded a yellow solid, 97\%, mp $281^{\circ} \mathrm{C}$ (Lit $\left.283^{\circ} \mathrm{C}\right) .^{[1]}$

${ }^{1} \mathrm{H}$ NMR (DMSO): 8.25 (2H, t, $\left.J=5.5 \mathrm{~Hz}\right), 7.91(2 \mathrm{H}, \mathrm{s}), 7.53(2 \mathrm{H}, \mathrm{d}, J=1.9 \mathrm{~Hz}), 7.26(2 \mathrm{H}, \mathrm{dd}, J=8.3,1.9 \mathrm{~Hz})$, 6.85 (2H, d, J = 8.3 Hz), 3.20 (4H, br s), 1.49 (4H, br s).

${ }^{13}$ C NMR (DMSO): 161.5, 150.9, 150.4, 145.7, 125.2, 123.1, 117.2, 115.8, 100.5, 39.3.

2-Cyano-N-\{3-[2-cyano-3-(3,4,5-trihydroxyphenyl)acryloylamino]butyl\}-3-(3,4,5-trihydroxyphenyl)acrylamide (36).

Synthesized as per $\mathbf{9}$ from 2-cyano- $N$-[3-(2-cyanoacetylamino)butyl]acetamide $\quad(5 \mathbf{c})$ and 3,4,5trihydroxybenzaldehyde. Afforded a yellow solid, $82 \%$, $\mathrm{mp}>300^{\circ} \mathrm{C}$ (Lit $\left.>310^{\circ} \mathrm{C}\right) .^{[1]}$

${ }^{1} \mathrm{H}$ NMR (DMSO): 8.16 (2H, t, $\left.J=5.5 \mathrm{~Hz}\right), 7.78$ (2H, s), 6.98 (4H, s), 3.19 (4H, br s), 1.48 (4H, br s).

${ }^{13}$ C NMR (DMSO): 161.7, 150.6, 145.9, 140.2, 121.3, 117.3, 109.9, 99.8, 39.3, 26.4.

2-Cyano- $N$-[3-(2-cyanoacetylamino)pentyl]acetamide (5d).

Synthesized as per 5a from 1,5-diaminopentane (3d) and methylcyanoacetate (4). Afforded a white solid, 98\%, mp $125^{\circ} \mathrm{C}\left(\right.$ Lit $\left.125^{\circ} \mathrm{C}\right) .^{[1]}$

${ }^{1} \mathrm{H}$ NMR (DMSO): 8.14 (2H, t, $J=5.4 \mathrm{~Hz}$ ), 3.55 (s, 4H), 3.03 (4H, q, $J=6.4 \mathrm{~Hz}$ ), 1.39 (4H, quin, $\left.J=7 \mathrm{~Hz}\right), 1.23$ (2H, quin, $J=7 \mathrm{~Hz})$.

${ }^{13} \mathrm{C}$ NMR (DMSO): 161.8, 116.1, 38.8, 28.3, 25.2, 23.4.

2-Cyano- $N$-\{3-[2-cyano-3-(3,4-dihydroxyphenyl)acryloylamino]pentyl\}-3-(3,4-dihydroxyphenyl)acrylamide (48). Synthesized as per $\mathbf{9}$ from 2-cyano- $N$-[3-(2-cyanoacetylamino)pentyl]acetamide (5d) and 3,4dihydroxybenzaldehyde. Afforded a yellow solid, $90 \%$, mp $252^{\circ} \mathrm{C}\left(\right.$ Lit $\left.248^{\circ} \mathrm{C}\right) .^{[1]}$

${ }^{1} \mathrm{H}$ NMR (DMSO): 8.15 (2H, t, $\left.J=5.5 \mathrm{~Hz}\right), 7.85(2 \mathrm{H}, \mathrm{s}), 7.50(2 \mathrm{H}, \mathrm{d}, J=2.1 \mathrm{~Hz}), 7.20(2 \mathrm{H}, \mathrm{dd}, J=8.5 \mathrm{~Hz}, 2 \mathrm{~Hz})$, $6.75(2 \mathrm{H}, \mathrm{d}, J=8.5 \mathrm{~Hz}), 3.16(4 \mathrm{H}, \mathrm{q}, J=6.2 \mathrm{~Hz}), 1.50$ (4H, quin, $J=7.1 \mathrm{~Hz}), 1.28$ (2H, quin, $J=6.9 \mathrm{~Hz})$.

${ }^{13}$ C NMR (DMSO): 161.9, 153.9, 150.3, 146.3, 126.2, 121.5, 117.7, 115.7, 114.7, 98.4, 39.5, 28.6, 23.7 (49).

2-Cyano- $N$-\{3-[2-cyano-3-(3,4,5-trihydroxyphenyl)acryloylamino]pentyl\}-3-(3,4,5-trihydroxyphenyl)acrylamide

Synthesized as per $\mathbf{9}$ from 2-cyano- $N$-[3-(2-cyanoacetylamino)pentyl]acetamide $\quad$ (5d) and 4,5trihydroxybenzaldehyde. Afforded a yellow solid, $83 \%, \mathrm{mp}>300^{\circ} \mathrm{C}$ (Lit $\left.>300^{\circ} \mathrm{C}\right) .{ }^{[2]}$

${ }^{1} \mathrm{H}$ NMR (DMSO): 8.12 (2H, t, $\left.J=5.5 \mathrm{~Hz}\right), 7.76(2 \mathrm{H}, \mathrm{s}), 6.98(4 \mathrm{H}, \mathrm{s}), 3.16(4 \mathrm{H}, \mathrm{br}$ s), 1.50 (4H, quin, $J=6.8$ $\mathrm{Hz}$ ), 1.28 (2H, quin, $J=6.7 \mathrm{~Hz}$ ).

${ }^{13}$ C NMR (DMSO): 161.8, 150.5, 146.1, 146.0, 141.3, 120.7, 117.5, 109.9, 99.1, 39.5, 28.6, 22.3. 
2-Cyano- $N$-[3-(2-cyanoacetylamino)hexyl]acetamide (5e).

Synthesized as per 5a from 1,6-diaminohexane (3e) and methylcyanoacetate (4). Afforded a white solid, 95\%, mp $141^{\circ} \mathrm{C}\left(\right.$ Lit $\left.140^{\circ} \mathrm{C}\right) .{ }^{[1]}$

${ }^{1} \mathrm{H}$ NMR (DMSO): 8.15 (2H, t, $J=5.5 \mathrm{~Hz}$ ), $3.56(4 \mathrm{H}, \mathrm{s}), 3.04$ (4H, q, $\left.J=6.1 \mathrm{~Hz}\right), 1.37$ (4H, quin, $J=5.9 \mathrm{~Hz}$ ), $1.24(4 \mathrm{H}, \mathrm{br} \mathrm{s})$.

${ }^{13}$ C NMR (DMSO): 161.8, 116.1, 38.9, 28.6, 25.8, 25.2.

2-Cyano-N-\{3-[2-cyano-3-(3,4-dihydroxyphenyl)acryloylamino] hexyl\}-3-(3,4-dihydroxyphenyl)acrylamide (61).

Synthesized as per $\mathbf{9}$ from 2-cyano- $N$-[3-(2-cyanoacetylamino)hexyl]acetamide (5e) and 3,4dihydroxybenzaldehyde. Afforded a yellow solid, $89 \%$, mp $263^{\circ} \mathrm{C}\left(\right.$ Lit $\left.260{ }^{\circ} \mathrm{C}\right) .^{[1]}$

${ }^{1} \mathrm{H}$ NMR (DMSO): 8.18 (2H, t, $\left.J=5.5 \mathrm{~Hz}\right), 7.89$ (2H, s), 7.51 (2H, d, $\left.J=2 \mathrm{~Hz}\right), 7.24$ (2H, dd, $J=2 \mathrm{~Hz}, 8.3 \mathrm{~Hz}$ ), $6.83(2 \mathrm{H}, \mathrm{d}, J=8.3 \mathrm{~Hz}), 3.17(4 \mathrm{H}, \mathrm{q}, J=6.1 \mathrm{~Hz}), 1.47(4 \mathrm{H}$, quin, $J=6.1 \mathrm{~Hz}), 1.28(4 \mathrm{H}, \mathrm{br} \mathrm{s})$.

${ }^{13}$ C NMR (DMSO): 161.5, 151.2, 150.3, 145.7, 125.2, 122.9, 117.2, 115.8, 115.7, 100.4, 39.5, 28.8, 26.0.

2-Cyano-N-[2-(2-cyanoacetylamino)cyclohexyl]acetamide (5h).

Synthesised as per $\mathbf{5 a}$ from 1,2-diaminocyclohexane (3h) and methylcyanoacetate (4). Afforded a white solid, $40 \%$, mp $85^{\circ} \mathrm{C}\left(\right.$ Lit $\left.83^{\circ} \mathrm{C}\right) . .^{[1]}$

${ }^{1} \mathrm{H}$ NMR (DMSO): 8.25 (2H, t, $\left.J=5.5 \mathrm{~Hz}\right), 3.58(4 \mathrm{H}, \mathrm{s}), 3.32(2 \mathrm{H}, \mathrm{m}), 1.9(8 \mathrm{H}, \mathrm{m})$.

${ }^{13}$ C NMR (DMSO): 162.1, 115.0, 53.1, 31.2, 23.3, 24.4. (75).

2-Cyano-N-\{3-[2-cyano-3-(3,4-dihydroxyphenyl)acryloylamino] cyclohexyl\}-3-(3,4-dihydroxyphenyl)acrylamide

Synthesized as per $\mathbf{9}$ from 2-cyano-N-[2-(2-cyanoacetylamino)cyclohexyl]acetamide $\quad(\mathbf{5 h})$ and 3,4dihydroxybenzaldehyde. Afforded a yellow solid, $64 \%$, mp $275^{\circ} \mathrm{C}\left(\right.$ Lit $\left.275^{\circ} \mathrm{C}\right) .^{[1]}$

${ }^{1} \mathrm{H}$ NMR (DMSO): 8.17 (2H, t, $\left.J=5.5 \mathrm{~Hz}\right), 7.94(2 \mathrm{H}, \mathrm{s}), 7.54(2 \mathrm{H}, \mathrm{d}, J=2.1 \mathrm{~Hz}), 7.35(2 \mathrm{H}, \mathrm{dd} J=8.2 \mathrm{~Hz}, 2.1$ $\mathrm{Hz}), 6.90(2 \mathrm{H}, \mathrm{d} J=8.2 \mathrm{~Hz}), 3.35(2 \mathrm{H}, \mathrm{m}), 1.81(8 \mathrm{H}, \mathrm{m})$.

${ }^{13}$ C NMR (DMSO): 162.1, 151.6, 150.0, 145.8, 125.2, 122.6, 117.1, 115.8, 115.4, 100.4, 53.1, 31.2, 24.4.

2-Cyano- $N$-\{3-[(2-cyano-acetylamino)methy]benzyl\}acetamide(5i)

Synthesized as per $\mathbf{5 a}$ from $m$-xylenediamine (3i) and methylcyanoacetate (4). Afforded a white solid, 70\%, mp $172^{\circ} \mathrm{C}\left(\right.$ Lit $\left.170^{\circ} \mathrm{C}\right) \cdot{ }^{[1]}$

${ }^{1} \mathrm{H}$ NMR (DMSO): 8.25 (2H, t, $\left.J=5.5 \mathrm{~Hz}\right), 7.30(4 \mathrm{H}, \mathrm{m}), 3.65(2 \mathrm{H}, \mathrm{s}), 4.43(4 \mathrm{H}, \mathrm{m})$

${ }^{13}$ C NMR (DMSO): 162.1, 138.6, 128.4, 126.3, 126.0, 116.1, 42.5, 25.2.

2-Cyano- $N$-\{3-[2-cyano-3-(3,4-dihydroxyphenylacryloylamino]methyl\}benzyl)-3-(3,4-

dihydroxyphenyl)acrylamide (76).

Synthesized as per 9 from 2-cyano-N-\{3-[(2-cyanoacetylamino)methy]benzyl\}acetamide (5i) and 3,4dihydroxybenzaldehyde. Afforded a yellow solid, $45 \%$, mp $245^{\circ} \mathrm{C}\left(\right.$ Lit $\left.246{ }^{\circ} \mathrm{C}\right) .^{[1]}$

${ }^{1} \mathrm{H}$ NMR (DMSO): 8.52 (2H, t, $\left.J=5.5 \mathrm{~Hz}\right), 7.94$ (2H, s) 7.54 (2H, d J= 2.2), 7.30 (6H, m), 6.93 (2H, d J=8.2 Hz), $4.43(4 \mathrm{H}, \mathrm{m})$

${ }^{13}$ C NMR (DMSO): 161.9, 152.1, 150.6, 145.8, 139.2, 128.2, 126.4, 125.9, 125.7, 122.5, 117.3, 115.8, 115.4, 99.4, 45.2, 21.7.

\section{2-Cyano-N-[3-(2-cyanoacetylamino)-2-hydroxypropyl]acetamide (5j)}

Synthesized as per 5a from 2-hydroxy-1,3-diaminopropane (3j) and methylcyanoacetate (4). Afforded a white solid, $90 \%$, mp $180^{\circ} \mathrm{C}$.

${ }^{1} \mathrm{H}$ NMR (DMSO): 8.2 (2H, t, $\left.J=5.1 \mathrm{~Hz}\right), 5.10$ (OH, s br), 3.61 (4H, s), $3.14(2 \mathrm{H}, \mathrm{br}), 3.11(2 \mathrm{H}, \mathrm{m}),(2 \mathrm{H}, \mathrm{m})$.

${ }^{13}$ C NMR (DMSO): 162.3, 116.2, 67.5, 43.2, 25.2.

Cyanoacetic acid 2-(2-cyanoacetoxy)ethyl ester (89a)

Ethylene glycol (88a) (1.56 g, $25 \mathrm{mmol})$, methylcyanoacetate (4) $(5.00 \mathrm{~g}, 50 \mathrm{mmol})$ and titanium (IV) butoxide $(0.2 \mathrm{~g}, 0.6 \mathrm{mmol})$ were heated at $100^{\circ} \mathrm{C}$ for $12 \mathrm{hrs}$. The mixture was allowed to cool, chloroform added $(50 \mathrm{~mL})$ and the resultant mixture filtered through a $10 \mathrm{~mm}$ pad of alumina. The solvent was removed in vacuo giving yellow oil. Cooling to $-10^{\circ} \mathrm{C}$ gave a white solid which was washed with ether and collected via filtration, $71 \%, 3.5 \mathrm{~g}, \mathrm{mp} 35^{\circ} \mathrm{C}$ $\left(\right.$ Lit $\left.35^{\circ} \mathrm{C}\right) .^{[3]}$

${ }^{1} \mathrm{H}$ NMR (DMSO): 4.10 (4H, t $\left.J=6.5 \mathrm{~Hz}\right), 3.95(4 \mathrm{H}, \mathrm{s}), 1.66(4 \mathrm{H}, \mathrm{t} \mathrm{J}=6.5 \mathrm{~Hz})$

${ }^{13}$ C NMR (DMSO): 164.2, 114.9, 65.2, 24.3 
Cyanoacetic acid 3-(2-cyanoacetoxy)propyl ester (89b)

Synthesized as per 89a from 1,3-propane diol (88b), methylcyanoacetate (4) and titanium (IV) butoxide. Afforded a white solid, $72 \%$, mp $37^{\circ} \mathrm{C}$ (Lit $\left.37^{\circ} \mathrm{C}\right) .^{[3]}$

${ }^{1} \mathrm{H}$ NMR (DMSO): $4.13(4 \mathrm{H}, \mathrm{t} J=6.5 \mathrm{~Hz}), 3.97(4 \mathrm{H}, \mathrm{s}), 1.59(4 \mathrm{H}, \mathrm{t} \mathrm{J}=6.5 \mathrm{~Hz}), 1.33(2 \mathrm{H}$, quin $J=3.75)$.

${ }^{13}$ C NMR (DMSO):164.2, 114.9, 65.6, 27.6, 24.3.

Cyanoacetic acid 4-(2-cyanoacetoxy)butyl ester (89c)

Synthesized as per 89a from 1,4-butanediol (88c), methylcyanoacetate (4) and titanium (IV) butoxide. Afforded a white solid, $58 \%$, mp $45^{\circ} \mathrm{C}\left(\right.$ Lit. $\left.43^{\circ} \mathrm{C}\right) .^{[3]}$

${ }^{1} \mathrm{H}$ NMR (DMSO): 4.13 (4H, t $\left.J=6.5 \mathrm{~Hz}\right), 3.97(4 \mathrm{H}, \mathrm{s}), 1.59(4 \mathrm{H}, \mathrm{t} \mathrm{J}=6.5 \mathrm{~Hz})$

${ }^{13}$ C NMR (DMSO):164.2, 114.9, 65.3, 27.9, 24.2

2-Cyano-N-[2-(2-cyano-3-phenylacryloylamino)ethyl]3-phenylacrylamide (6).

2-Cyano- $N$-[3-(2-cyanoacetylamino)ethyl]acetamide (5a) $(0.3 \mathrm{~g}, 1.5 \mathrm{mmol})$, benzaldehyde $(0.33 \mathrm{~g}, 3 \mathrm{mmol}), 3$ drops of piperidine and ethanol $(10 \mathrm{~mL})$ were refluxed for 2 hours. Cooling, filtering and washing with cold ether $(10 \mathrm{~mL})$ gave a yellow solid, $79 \%, 0.45 \mathrm{~g}$. $\mathrm{mp} 178-180^{\circ}$

${ }^{1} \mathrm{H}$ NMR (DMSO): 8.57 (2H, br t), 8.17 (2H, s), 7.92 (4H, m), 7.57 (6H, m), 3.42 (4H, br s).

${ }^{13}$ C NMR (DMSO): 161.2, 150.5, 132.2, 131.3, 129.9, 129.1, 116.3, 106.4, 38.9.

2-Cyano-N-\{2-[2-cyano-3-(3-hydroxyphenyl)acryloylamino]ethyl\}-3-(3-hydroxyphenyl)acrylamide (7).

Synthesized as per $\mathbf{6}$ from 2-cyano- $N$-[3-(2-cyanoacetylamino)ethyl]acetamide (5a) and 3-hydroxybenzaldehyde. Afforded a yellow solid, $0.53 \mathrm{~g}(85 \%) \mathrm{mp} 290-292^{\circ} \mathrm{C}$

${ }^{1} \mathrm{H}$ NMR (DMSO): 8.54 (2H, br t), 8.04 (2H, s), 7.36 (4H, m), 7.32 (2H, s), 6.97 (2H, m), 3.38 (4H br s).

${ }^{13}$ C NMR (DMSO): 161.8, 158.2, 151.0, 133.5, 10.7, 121.8, 120.1, 116.7, 116.2, 106.5, 39.6.

2-Cyano- $N$-\{2-[2-cyano-3-(4-hydroxyphenyl)acryloylamino]ethyl\}-3-(4-hydroxyphenyl)acrylamide (8).

Synthesized as per $\mathbf{6}$ from 2-cyano- $N$-[3-(2-cyanoacetylamino)ethyl]acetamide (5a) and 4-hydroxybenzaldehyde. Afforded a bright yellow solid, $78 \%$. $\mathrm{mp}>300^{\circ} \mathrm{C}$

${ }^{1} \mathrm{H}$ NMR (DMSO): 8.36 (2H, br t), 8.02 (2H, s), 7.85 (4H, d $J=7.2 \mathrm{~Hz}$ ), 6.89 (4H, d $J=8.8 \mathrm{~Hz}$ ), 3.36 (4H, br s).

${ }^{13}$ C NMR (DMSO): 162.3, 150.8, 133.2, 123.2, 117.6, 116.7, 101.5, 39.6.

3-(2-Chloro-phenyl)- $N$-\{2-[3-(2-chlorophenyl)-2-cyanoacryloylamino]ethyl\}-2-cyanoacrylamide (12).

Synthesized as per 6 from 2-cyano- $N$-[3-(2-cyanoacetylamino)ethyl]acetamide (5a) and 2-chlorobenzaldehyde. Afforded a light yellow solid, $83 \%$. $\mathrm{mp} 248-250^{\circ} \mathrm{C}$

${ }^{1} \mathrm{H}$ NMR (DMSO): 8.70 (2H, br t), 8.36 (2H, s), 8.00 (2H, dd $\left.J=6.2 \mathrm{~Hz}, J J=2.1 \mathrm{~Hz}\right), 7.62,(2 \mathrm{H}, \mathrm{m}), 7.54(4 \mathrm{H}$, $\mathrm{m}), 3.41$ ( $4 \mathrm{H}, \mathrm{br} \mathrm{s})$

${ }^{13}$ C NMR (DMSO): 160.4, 146.8, 134.0, 133.2, 130.3, 130.0, 129.5, 127.7, 115.3, 110.4, 39.1.

3-(4-Chlorophenyl)- $N$-\{2-[3-(4-chlorophenyl)-2-cyanoacryloylamino]ethyl\}-2-cyanoacrylamide (13).

Synthesized as per 6 from 2-cyano- $N$-[3-(2-cyanoacetylamino)ethyl]acetamide (5a) and 4-chlorobenzaldehyde. Afforded a yellow solid, $76 \%$. mp $244-246^{\circ} \mathrm{C}$.

${ }^{1} \mathrm{H}$ NMR (DMSO): 8.58 (2H, br t), 8.16 (2H, s), 7.91 (4H, d $J=8.6 \mathrm{~Hz}$ ), 7.63 (4H, d $J=6.8 \mathrm{~Hz}$ ), 3.32 (4H, br s).

${ }^{13}$ C NMR (DMSO): 161.0, 149.1, 136.8, 131.6, 130.7, 129.3, 116.0, 106.9, 39.2.

2-Cyano- $N$-\{2-[2-cyano-3-(3-methoxyphenyl)acryloylamino]ethyl\}-3-(3-methoxyphenyl)acrylamide (14).

Synthesized as per $\mathbf{6}$ from 2-cyano- $N$-[3-(2-cyanoacetylamino)ethyl]acetamide (5a) and 3-methoxybenzaldehyde. Afforded a light yellow solid, $65 \%$. $\mathrm{mp} 118-120^{\circ} \mathrm{C}$

${ }^{1} \mathrm{H}$ NMR (DMSO): 8.57 (2H, br t), 8.14 (2H, s), 7.49 (2H, m), 7.47 (2H, m), 7.15 (4H, m), 3.79 (6H, s), 3.98 (4H, br s)

${ }^{13}$ C NMR (DMSO): 161.2, 159.4, 150.4, 133.1, 130.3, 122.3, 118.1, 116.3, 114.7, 106.5, 55.2, 39.4. 
2-Cyano-N-\{2-[2-cyano-3-(4-methoxyphenyl)acryloylamino]ethyl\}-3-(4-methoxyphenyl)acrylamide (15).

Synthesized as per 6 from 2-cyano- $N$-[3-(2-cyanoacetylamino)ethyl]acetamide (5a) and 4-methoxybenzaldehyde. Afforded a light yellow solid, $94 \% \mathrm{mp} 246-248{ }^{\circ} \mathrm{C}$.

${ }^{1} \mathrm{H}$ NMR (DMSO): 8.43 (2H, br t), 8.09 (2H, s), 7.95 (4H, d $\left.J=8.9 \mathrm{~Hz}\right), 7.12$ (4H, d $\left.J=8.9 \mathrm{~Hz}\right), 3.84$ (6H, s), $3.36(4 \mathrm{H}$, br s)

${ }^{13}$ C NMR (DMSO): 163.0, 162.1, 150.5, 132.8, 124.8, 117.4, 115.2, 103.1, 56.0, 40.2

2-Cyano- $N$-\{2-[2-cyano-3-(3,4-dimethoxyphenyl)acryloylamino] ethyl\}-3-(3,4-dimethoxyphenyl)acrylamide (16).

Synthesized as per $\mathbf{6}$ from 2-cyano- $N$-[3-(2-cyanoacetylamino)ethyl]acetamide (5a) and 3,4dimethoxybenzaldehyde. Afforded a light yellow solid, $69 \% . \mathrm{mp}>300^{\circ} \mathrm{C}$

${ }^{1} \mathrm{H}$ NMR (DMSO): 8.43 (2H, t $\left.J=5.5 \mathrm{~Hz}\right), 8.08$ (2H, s), 7.65 (2H, d $\left.J=1.9 \mathrm{~Hz}\right), 7.55$ (2H, dd $J=9.0 \mathrm{~Hz}, J J=2$ $\mathrm{Hz}), 7.14$ (2H, d $J=8.6 \mathrm{~Hz}), 3.84$ (6H, s), 3.77 (6H, s), 3.31(4H, br s).

${ }^{13}$ C NMR (DMSO): 162.1, 152.9, 150.9, 149.1, 125.8, 124.9, 117.5, 112.7, 112.3, 103.0, 56.2, 55.9, 40.2.

2-Cyano-N-\{2-[2-cyano-3-(3-hydroxy-4-methoxyphenyl)acryloylamino]ethyl\}-3-(3-hydroxy-4methoxyphenyl)acrylamide (17).

Synthesized as per $\mathbf{6}$ from 2-cyano- $N$-[3-(2-cyanoacetylamino)ethyl]acetamide (5a) and 3-hydroxy-4methoxybenzaldehyde. Afforded a bright yellow solid, $98 \% \mathrm{mp} 274-276^{\circ} \mathrm{C}$.

${ }^{1} \mathrm{H}$ NMR (DMSO): 8.40 (2H, br t), 7.97 (2H, s), 7.53 (2H, d $J=2.0 \mathrm{~Hz}$ ), 7.37 (4H, dd $J=8.6 \mathrm{~Hz}, J J=2.1 \mathrm{~Hz}$ ), 7.06 (4H, d $J=8.6 \mathrm{~Hz}), 3.84$ (6H, s), 3.36 (4H, br s).

${ }^{13}$ C NMR (DMSO): 161.8, 151.7, 150.3, 146.7, 124.5, 116.8, 115.4, 112.0, 102.2, 55.6, 39.1.

2-Cyano-N-\{2-[2-cyano-3-(4-carboxyphenyl)acryloylamino]ethyl\}-3-(4-carboxyphenyl)acrylamide (18).

Synthesized as per $\mathbf{6}$ from 2-cyano- $N$-[3-(2-cyanoacetylamino)ethyl]acetamide (5a) and 4-carboxybenzaldehyde. Afforded a white solid, $96 \%$. $\mathrm{mp}>300^{\circ} \mathrm{C}$

${ }^{1} \mathrm{H}$ NMR (DMSO): 8.68 (2H, t $J=5.3 \mathrm{~Hz}$ ), $8.21(2 \mathrm{H}, \mathrm{s}), 8.00$ (4H, d $J=8.0 \mathrm{~Hz}$ ), 7.97 (4H, d $\left.J=8.1 \mathrm{~Hz}\right), 3.40$ (4H, br s).

${ }^{13}$ C NMR (DMSO): 166.7, 162.4, 161.0, 149.4, 135.3, 134.3, 129.8, 129.8, 116.1, 115.9, 108.2.

2-Cyano-N-[3-(2-cyano-3-phenylacryloylamino)propyl]-3-phenylacrylamide (19).

Synthesized as per $\mathbf{6}$ from 2-cyano- $N$-[3-(2-cyanoacetylamino)propyl]acetamide (5b) and benzaldehyde. Afforded a yellow solid, $81 \%$. mp $138-140^{\circ} \mathrm{C}$

${ }^{1} \mathrm{H}$ NMR (DMSO): 8.37 (2H, s), 7.95 (4H, d $\left.J=6.8 \mathrm{~Hz}\right), 7.52(6 \mathrm{H}, \mathrm{m}) 7.12$ (2H, br t), 3.53 (4H, q $\left.J=6.0 \mathrm{~Hz}\right), 1.7$ (2H, br quin).

${ }^{13}$ C NMR (DMSO): 161.1, 153.2, 132.7, 131.7, 130.6, 129.2, 116.8, 103.8, 37.1, 29.5.

2-Cyano- $N$-\{3-[2-cyano-3-(3-hydroxyphenyl)acryloylamino]propyl\}-3-(3-hydroxyphenyl)acrylamide (20).

Synthesized as per $\mathbf{6}$ from 2-cyano- $N$-[3-(2-cyanoacetylamino)propyl]acetamide $\quad(5 \mathbf{b})$ and 3hydroxybenzaldehyde. Afforded a yellow solid, $71 \% \mathrm{mp} 212-216^{\circ} \mathrm{C}$.

${ }^{1} \mathrm{H}$ NMR (DMSO): 8.43 (t, 2H $J=5.5 \mathrm{~Hz}$ ), 8.05 (s, 2H), 7.35 (4H, m), 7.33 (2H, m). 6.96 (2H, m), 3.26 (4H, q $J$ $=6.1 \mathrm{~Hz}), 1.747(4 \mathrm{H}$, br quin).

${ }^{13}$ C NMR (DMSO): 161.4, 158.2, 151.0, 133.5, 130.7, 121.8, 120.1, 116.7, 116.2, 106.3, 37.9, 29.1.

2-Cyano-N-\{3-[2-cyano-3-(4-hydroxyphenyl)acryloylamino]propyl\}-3-(4-hydroxyphenyl)acrylamide (21).

Synthesized as per $\mathbf{6}$ from 2-cyano- $N$-[3-(2-cyanoacetylamino)propyl]acetamide $\quad(5 \mathbf{b})$ and 4 hydroxybenzaldehyde. Afforded a yellow solid, $58 \% \mathrm{mp} 250-252^{\circ} \mathrm{C}$.

${ }^{1} \mathrm{H}$ NMR (DMSO): 8.28 (2H, t $J=5.5 \mathrm{~Hz}$ ), 8.03 (2H, s), 7.85 (4H, d $\left.J=8.8 \mathrm{~Hz}\right), 6.90$ (4H, d $J=8.7 \mathrm{~Hz}$ ), 3.23 $(4 \mathrm{H}$, q $J=6.1 \mathrm{~Hz}), 1.71(2 \mathrm{H}$, quin $J=6.7 \mathrm{~Hz})$.

${ }^{13}$ C NMR (DMSO): 162.2, 161.9, 150.8, 133.3, 123.3 117.6, 116.6, 101.4, 37.8, 29.2. 
3-(2-Chlorophenyl)-N-\{3-[3-(2-chlorophenyl)-2-cyanoacryloylamino]propyl\}-2-cyanoacrylamide (25).

Synthesized as per $\mathbf{6}$ from 2-cyano- $N$-[3-(2-cyanoacetylamino)propyl]acetamide (5b) and 2-chlorobenzaldehyde. Afforded a yellow solid, $80 \% \mathrm{mp} 172-174^{\circ} \mathrm{C}$.

${ }^{1} \mathrm{H}$ NMR (DMSO): 8.57 (2H, br t), 8.36 (2H, s), 7.98 (2H, dd $\left.J=4.1 \mathrm{~Hz}, 2.1 \mathrm{~Hz}\right), 7.52$ (6H, m), 3.32 (4H, q $J=$ $6.0 \mathrm{~Hz}), 1.77$ (2H, br quin).

${ }^{13}$ C NMR (DMSO): 159.9, 146.8, 134.0, 133.2, 130.3, 130.0, 129.6, 127.7, 115.3, 110.3, 37.6, 28.4.

3-(4-Chlorophenyl)- $N$-\{3-[3-(4-chlorophenyl)-2-cyanoacryloylamino]propyl\}-2-cyanoacrylamide (26).

Synthesized as per $\mathbf{6}$ from 2-cyano- $N$-[3-(2-cyanoacetylamino)propyl]acetamide (5b) and 4-chlorobenzaldehyde. Afforded a yellow solid, $87 \% \mathrm{mp} 168-172^{\circ} \mathrm{C}$

${ }^{1} \mathrm{H}$ NMR (DMSO): 8.47 (2H, t $J=5.3 \mathrm{~Hz}$ ), 8.15 (2H, s), 7.92 (4H, d $J=8.4 \mathrm{~Hz}$ ), 7.60 (4H, d $J=8.4 \mathrm{~Hz}$ ), 3.29 $(4 \mathrm{H}, \mathrm{q} J=5.9 \mathrm{~Hz}), 1.76(2 \mathrm{H}$, quin $J=6.5 \mathrm{~Hz})$.

${ }^{13}$ C NMR (DMSO): 160.6, 149.1, 136.8, 131.6, 130.7, 129.2, 116.1, 106.8, 37.5, 28.5.

2-Cyano- $N$-\{3-[2-cyano-3-(3-methoxyphenyl)acryloylamino] propyl\}-3-(3-methoxyphenyl)acrylamide (27).

Synthesized as per $\mathbf{6}$ from 2-cyano- $N$-[3-(2-cyanoacetylamino)propyl]acetamide $\quad(5 \mathbf{b})$ and 3methoxybenzaldehyde. Afforded a yellow solid, $59 \% \mathrm{mp} 132-134^{\circ} \mathrm{C}$.

${ }^{1} \mathrm{H}$ NMR (DMSO): 8.46 (2H, br t), 8.14, (1H, s), 7.50 (2H, s), 7.44 (m, 4H), 7.13 (2H, m), 3.78 (s, 6H), $3.29(4 \mathrm{H}$, q), 1.77 (2H, quin).

${ }^{13}$ C NMR (DMSO): 160.8, 159.3, 150.4, 133.1, 130.2, 122.3, 118.1, 116.3, 106.4, 55.2, 37.5, 28.5.

2-Cyano- $N$-\{3-[2-cyano-3-(4-methoxyphenyl)acryloylamino]propyl\}-3-(4-methoxyphenyl)acrylamide (28).

Synthesized as per $\mathbf{6}$ from 2-cyano- $N$-[3-(2-cyanoacetylamino)propyl]acetamide $\quad \mathbf{5 b}$ ) and 4 methoxybenzaldehyde. Afforded a yellow solid, $88 \%$. $\mathrm{mp} 190-192^{\circ} \mathrm{C}$

${ }^{1} \mathrm{H}$ NMR (DMSO): 8.31 (t, 2H), 8.08 (s, 2H), 7.93 (4H, d $J=8.6 \mathrm{~Hz}$ ), 7.10 (4H, d $J=8.6 \mathrm{~Hz}$ ), 3.83 (6H, s), 3.27 $(4 \mathrm{H}, \mathrm{q} J=5.8 \mathrm{~Hz}), 1.74(2 \mathrm{H}$, quin $J=6.3 \mathrm{~Hz})$.

${ }^{13}$ C NMR (DMSO): 162.5, 161.2, 150.0, 132.4, 124.3, 116.9, 114.7, 102.4, 55.5, 37.4, 28.7. (29).

Synthesized as per $\mathbf{6}$ from 2-cyano- $N$-[3-(2-cyanoacetylamino)propyl]acetamide $\quad(\mathbf{5 b})$ and 3,4dimethoxybenzaldehyde. Afforded a yellow solid, $93 \% \mathrm{mp}>300^{\circ} \mathrm{C}$.

${ }^{1} \mathrm{H}$ NMR (DMSO): 8.30 (t, $\left.2 \mathrm{H} J=5.5 \mathrm{~Hz}\right), 8.07(2 \mathrm{H}, \mathrm{s}), 7.62(2 \mathrm{H}, \mathrm{d} J=1.9 \mathrm{~Hz}$ ), 7.55 (2H, dd $J=9.0 \mathrm{~Hz}, J J=2$ $\mathrm{Hz}), 7.10(2 \mathrm{H}, \mathrm{d} J=8.6 \mathrm{~Hz}), 3.83(6 \mathrm{H}, \mathrm{s}), 3.78(6 \mathrm{H}, \mathrm{s}), 3.28(4 \mathrm{H}, \mathrm{q}), 1.74$ (2H, quin).

${ }^{13}$ C NMR (DMSO): 161.2, 152.4, 150.4, 148.5, 125.4, 124.4, 117.0, 112.1, 111.6, 102.3, 55.6, 55.3, 37.4, 28.6.

2-Cyano-N-\{3-[2-cyano-3-(3-hydroxy-4-methoxyphenyl)acryloylamino]propyl\}-3-(3-hydroxy-4methoxyphenyl)acrylamide (30).

Synthesized as per 6 from 2-cyano- $N$-[3-(2-cyanoacetylamino)propyl]acetamide (5b) and 3-hydroxy-4methoxybenzaldehyde. Afforded a yellow solid, 50\%. mp 194-196 ${ }^{\circ} \mathrm{C}$.

${ }^{1} \mathrm{H}$ NMR (DMSO): 8.28 (t, 2H $\left.J=5.1 \mathrm{~Hz}\right), 7.98(2 \mathrm{H}, \mathrm{s}), 7.53(2 \mathrm{H}, \mathrm{d} J=1.6 \mathrm{~Hz}$ ), $7.36(2 \mathrm{H}, \mathrm{dd} J=8.3 \mathrm{~Hz}, 1.5$ $\mathrm{Hz}), 7.04$ (2H, d $J=8.5 \mathrm{~Hz}$ ), $3.84(6 \mathrm{H}, \mathrm{s}), 3.26(4 \mathrm{H}, \mathrm{q} J=5.6 \mathrm{~Hz}$ ), $1.73(2 \mathrm{H}$, quin $J=6.5 \mathrm{~Hz})$.

${ }^{13}$ C NMR (DMSO): 161.4, 151.7, 150.4, 146.7, 124.5, 124.5, 116.5, 114.5, 111.9, 102.1, 102.0, 55.6, 37.4, 28.7.

2-Cyano- $N$-\{3-[2-cyano-3-(4-carboxyphenyl)acryloylamino]propyl\}-3-(4-carboxyphenyl)acrylamide (31).

Synthesized as per $\mathbf{6}$ from 2-cyano- $N$-[3-(2-cyanoacetylamino)propyl]acetamide $\quad(5 \mathbf{b})$ and 4 carboxybenzaldehyde. Afforded a white solid, $82 \% \mathrm{mp} 260-264^{\circ} \mathrm{C}$.

${ }^{1} \mathrm{H}$ NMR (DMSO): 8.62 (2H, t), 8.31 (2H, s), 8.10 (4H, d $J=8.0 \mathrm{~Hz}$ ), 7.98 (4H, d $J=8.1 \mathrm{~Hz}$ ), 3.23 (4H, q $J=5.7$

$\mathrm{Hz}), 1.75(2 \mathrm{H}$, quin $J=6.6 \mathrm{~Hz}$ ). 
${ }^{13}$ C NMR (DMSO): 166.7, 162.4, 161.0, 149.4, 135.3, 134.3, 129.8, 129.8, 116.1, 115.9, 108.2, 39.4, 26.2.

2-Cyano-N-[4-(2-cyano-3-phenylacryloylamino)butyl]-3-phenylacrylamide (32).

Synthesized as per 6 from 2-cyano- $N$-[3-(2-cyanoacetylamino)butyl]acetamide (5c) and benzaldehyde. Afforded a yellow solid, $85 \% \mathrm{mp} 188-190^{\circ} \mathrm{C}$.

${ }^{1} \mathrm{H}$ NMR (DMSO): 8.51 (2H, t $\left.J=5.6 \mathrm{~Hz}\right), 7.94$ (2H, s), $7.92(4 \mathrm{H}, \mathrm{m}), 7.56(6 \mathrm{H}, \mathrm{m}), 3.23$ (4H, q $\left.J=5.6 \mathrm{~Hz}\right), 1.53$ (4H, br s).

${ }^{13}$ C NMR (DMSO): 160.8, 150.3, 132.2, 131.9, 129.9, 129.2, 116.3, 106.4, 39.3, 26.2.

2-Cyano- $N$-\{4-[2-cyano-3-(3-hydroxyphenyl)acryloylamino]butyl\}-3-(3-hydroxyphenyl)acrylamide (33).

Synthesized as per 6 from 2-cyano- $N$-[3-(2-cyanoacetylamino)butyl]acetamide (5c) and 3-hydroxybenzaldehyde. Afforded a yellow solid, $80 \% \mathrm{mp} 224-228{ }^{\circ} \mathrm{C}$.

${ }^{1} \mathrm{H}$ NMR (DMSO): 8.46 (2H, br t), 8.03 (2H, s), 7.36 (4H, m), 7.32 (2H, s), 6.96 (2H, m), 3.22 (4H, br s), 1.52 (4H, br s).

${ }^{13}$ C NMR (DMSO): 160.9, 157.7, 150.4, 133.0, 130.2, 121.3, 119.5, 116.3, 115.7, 106.0, 39.3, 26.2.

2-Cyano- $\mathrm{N}$-\{4-[2-cyano-3-(4-hydroxyphenyl)acryloylamino]butyl\}-3-(4-hydroxyphenyl)acrylamide (34).

Synthesized as per 6 from 2-cyano- $N$-[3-(2-cyanoacetylamino)butyl]acetamide (5c) and 4-hydroxybenzaldehyde. Afforded a yellow solid, $80 \% \mathrm{mp} 290-292^{\circ} \mathrm{C}$.

${ }^{1} \mathrm{H}$ NMR (DMSO): 8.28 (2H, t $\left.J=5.6 \mathrm{~Hz}\right), 8.01(2 \mathrm{H}, \mathrm{s}), 7.84(4 \mathrm{H}, \mathrm{d} J=8.9 \mathrm{~Hz}), 6.90(4 \mathrm{H}, \mathrm{d} J=8.76 \mathrm{~Hz}), 3.20$ $(4 \mathrm{H}, \mathrm{q} J=5.4 \mathrm{~Hz}), 1.49(4 \mathrm{H}, \mathrm{br}$ ) $)$.

${ }^{13}$ C NMR (DMSO): 161.7, 161.3, 150.2, 132.7, 122.8, 117.2, 116.1, 101.1, 39.3, 26.3.

3-(2-Chlorophenyl)- $\mathrm{N}$-\{4-[3-(2-chlorophenyl)-2-cyanoacryloylamino]butyl\}-2-cyanoacrylamide (38).

Synthesized as per 6 from 2-cyano- $N$-[3-(2-cyanoacetylamino)butyl]acetamide (5c) and 2-chlorobenzaldehyde. Afforded a yellow solid, $93 \% \mathrm{mp} 196-198^{\circ} \mathrm{C}$.

${ }^{1} \mathrm{H}$ NMR (DMSO): 8.61 (2H, t $\left.J=5.6 \mathrm{~Hz}\right), 7.98$ (2H, s), 7.63 (6H, dd $\left.J=4.2 \mathrm{~Hz}, 2.0 \mathrm{~Hz}\right), 7.56$ (6H, m), 3.25 (4H, q $J=5.6 \mathrm{~Hz}), 1.54$ (4H, br s).

${ }^{13}$ C NMR (DMSO): 159.9, 146.7, 134.0, 133.1, 130.3, 130.0, 129.6, 127.7, 115.3, 110.5.

3-(4-Chlorophenyl)- $\mathrm{N}$-\{4-[3-(4-chlorophenyl)-2-cyanoacryloylamino]butyl\}-2-cyanoacrylamide (39).

Synthesized as per 6 from 2-cyano- $N$-[3-(2-cyanoacetylamino)butyl]acetamide (5c) and 4-chlorobenzaldehyde. Afforded a yellow solid, $72 \% \mathrm{mp} 232-234^{\circ} \mathrm{C}$.

${ }^{1} \mathrm{H}$ NMR (DMSO): 8.47 (2H, br t), $8.14(2 \mathrm{H}, \mathrm{s}), 7.93$ (4H, d $\left.J=6.9 \mathrm{~Hz}\right), 7.62(4 \mathrm{H}, \mathrm{d} J=6.8 \mathrm{~Hz}), 3.23(4 \mathrm{H}, \mathrm{q} J=$ $5.5 \mathrm{~Hz}$ ), 1.53 (4H br s).

${ }^{13}$ C NMR (DMSO): 160.6, 149.0, 136.7, 131.6, 130.7, 129.3, 116.1, 107.0, 39.4, 26.2.

2-Cyano-N-\{4-[2-cyano-3-(3-methoxyphenyl)acryloylamino]butyl\}-3-(3-methoxyphenyl)acrylamide (40).

Synthesized as per 6 from 2-cyano- $N$-[3-(2-cyanoacetylamino)butyl]acetamide (5c) and 3-methoxybenzaldehyde. Afforded a yellow solid, $89 \% \mathrm{mp} 180-182^{\circ} \mathrm{C}$.

${ }^{1} \mathrm{H}$ NMR (DMSO): 8.46 (2H, t), 8.12 (2H, s), 7.45 (6H, m), 7.13 (2H, m), 3.78 (6H, s), 3.23 (4H, br s), 1.54 (4H, br s).

${ }^{13}$ C NMR (DMSO): 160.7, 159.3, 150.2, 133.1, 130.2, 122.3, 118.1, 116.3, 114.7, 106.6, 55.2, 39.3, 26.2.

2-Cyano-N-\{4-[2-cyano-3-(4-methoxyphenyl)acryloylamino]butyl\}-3-(4-methoxyphenyl)acrylamide (41).

Synthesized as per 6 from 2-cyano- $N$-[3-(2-cyanoacetylamino)butyl]acetamide (5c) and 4-methoxybenzaldehyde. Afforded a yellow solid, $81 \% \mathrm{mp} 200-202^{\circ} \mathrm{C}$. 
${ }^{1} \mathrm{H}$ NMR (DMSO): 8.32 (2H, t $J=5.6 \mathrm{~Hz}$ ), 8.07 (2H, s), 7.94 (4H, d $J=8.9 \mathrm{~Hz}$ ), 7.10 (4H, d $\left.J=8.9 \mathrm{~Hz}\right), 3.83$ $(6 \mathrm{H}, \mathrm{s}), 3.22(4 \mathrm{H}, \mathrm{q} J=5.5 \mathrm{~Hz}), 1.52(4 \mathrm{H}, \mathrm{br}$ s).

${ }^{13}$ C NMR (DMSO): 162.4, 161.2, 149.8, 132.3, 124.4, 116.9, 114.7, 102.7, 55.5, 39.2, 26.3.

2-Cyano- $N$-\{4-[2-cyano-3-(3,4-dimethoxyphenyl)acryloylamino] butyl\}-3-(3,4-dimethoxyphenyl)acrylamide (42).

Synthesized as per $\mathbf{6}$ from 2-cyano- $N$-[3-(2-cyanoacetylamino)butyl]acetamide $\quad$ (5c) and 3,4dimethoxybenzaldehyde. Afforded a yellow solid, $82 \%$. $\mathrm{mp}>300^{\circ} \mathrm{C}$

${ }^{1} \mathrm{H}$ NMR (DMSO): 8.32 (2H, t $J=5.5 \mathrm{~Hz}$ ), 8.07 (2H, s), $7.63(2 \mathrm{H}, \mathrm{d} J=1.9 \mathrm{~Hz}), 7.55$ (2H, dd $\left.J=8.3,1.9 \mathrm{~Hz}\right)$, $7.12(2 \mathrm{H}, \mathrm{d} J=8.3 \mathrm{~Hz}$ ), 3.84 (6H, s), 3.78 (6H, s), 3.22 (4H, br s), 1.52 (4H, br s).

${ }^{13}$ C NMR (DMSO): 161.2, 152.4, 150.2, 148.6, 125.3, 124.4, 117.1, 112.2, 111.7, 102.6, 55.7, 55.4, 39.3, 26.3.

2-Cyano-N-\{4-[2-cyano-3-(3-hydroxy-4-methoxyphenyl)acryloylamino]butyl\}-3-(3-hydroxy-4methoxyphenyl)acrylamide (43).

Synthesized as per $\mathbf{6}$ from 2-cyano- $N$-[3-(2-cyanoacetylamino)butyl]acetamide (5c) and 3-hydroxy-4methoxybenzaldehyde. Afforded a yellow solid, $91 \% \mathrm{mp} 294-296^{\circ} \mathrm{C}$.

${ }^{1} \mathrm{H}$ NMR (DMSO): 8.28 (2H, br t), 7.96 (2H, s), 7.52 (2H, d $\left.J=1.9 \mathrm{~Hz}\right), 7.37$ (2H, dd $J=8.5 \mathrm{~Hz}, 1.8 \mathrm{~Hz}$ ), 7.06 (2H, d $J=8.5 \mathrm{~Hz}), 3.84$ (6H, s), $3.21(2 \mathrm{H}, \mathrm{q} J=5.0 \mathrm{~Hz}), 1.51(2 \mathrm{H}, \mathrm{br}$ s).

${ }^{13}$ C NMR (DMSO): 161.3, 151.6, 150.1, 146.6, 124.5, 116.9, 115.4, 111.9, 102.2, 55.6, 39.3, 26.3.

2-Cyano- $N$-\{4-[2-cyano-3-(4-carboxyphenyl)acryloylamino]butyl\}-3-(4-carboxyphenyl)acrylamide (44).

Synthesized as per 6 from 2-cyano- $N$-[3-(2-cyanoacetylamino)butyl]acetamide (5c) and 4-carboxybenzaldehyde. Afforded a white solid, $50 \% \mathrm{mp} 298-300^{\circ} \mathrm{C}$.

${ }^{1} \mathrm{H}$ NMR (DMSO): 8.59 (2H, t $J=5.3 \mathrm{~Hz}$ ), 8.35 (4H, d $J=8.5 \mathrm{~Hz}$ ), 8.27 (2H, s), 8.10 (4H, d $\left.J=8.6 \mathrm{~Hz}\right), 3.25$ $(2 \mathrm{H}, \mathrm{q} J=4.9 \mathrm{~Hz}), 1.55(2 \mathrm{H}, \mathrm{br} \mathrm{s})$.

${ }^{13}$ C NMR (DMSO): 166.6, 160.5, 149.1, 135.5, 134.0, 129.8, 129.7, 116.0, 108.4, 39.5, 28.6.

2-Cyano-N-[5-(2-cyano-3-phenylacryloylamino)pentyl]-3-phenylacrylamide (45).

Synthesized as per 6 from 2-cyano- $N$-[3-(2-cyanoacetylamino)pentyl]acetamide (5d) and benzaldehyde. Afforded a yellow solid, $51 \% \mathrm{mp} 164-166^{\circ} \mathrm{C}$.

${ }^{1} \mathrm{H}$ NMR (DMSO): 8.43 (2H, t $J=5.3 \mathrm{~Hz}$ ), 8.14 (2H, s), 7.91(4H, m), 7.55 (6H, m), $3.21(4 \mathrm{H}, \mathrm{q} J=6.7 \mathrm{~Hz}), 1.52$ (4H, quin $J=7.3 \mathrm{~Hz}), 1.31(2 \mathrm{H}$, quin $J=6.7 \mathrm{~Hz})$.

${ }^{13}$ C NMR (DMSO): 160.8, 150.2, 132.1, 131.1, 129.9, 129.1, 116.3, 106.5, 39.5, 28.4, 23.7.

2-Cyano- $N$-\{5-[2-cyano-3-(3-hydroxyphenyl)acryloylamino]pentyl\}-3-(3-hydroxyphenyl)acrylamide (46).

Synthesized as per $\mathbf{6}$ from 2-cyano- $N$-[3-(2-cyanoacetylamino)pentyl]acetamide $\quad(\mathbf{5 d})$ and 3 hydroxybenzaldehyde. Afforded a yellow solid, $92 \% \mathrm{mp} 230-232^{\circ} \mathrm{C}$.

${ }^{1} \mathrm{H}$ NMR (DMSO): 8.42 (2H, t $J=5.4 \mathrm{~Hz}$ ), $8.02(2 \mathrm{H}, \mathrm{s}), 7.34(4 \mathrm{H}, \mathrm{m}), 7.31(2 \mathrm{H}, \mathrm{s}), 6.97(2 \mathrm{H}, \mathrm{m}), 3.19$ (4H, q $J=$ $6.0 \mathrm{~Hz}$ ), $1.52(4 \mathrm{H}$, quin $J=7.0 \mathrm{~Hz}), 1.32(2 \mathrm{H}$, quin $J=6.8 \mathrm{~Hz})$.

${ }^{13}$ C NMR (DMSO): 160.9, 157.7, 150.3, 133.0, 130.2, 121.3, 119.5, 116.3, 115.7, 106.1, 39.5, 28.4, 23.6.

2-Cyano-N-\{5-[2-cyano-3-(4-hydroxyphenyl)acryloylamino]pentyl\}-3-(4-hydroxyphenyl)acrylamide (47).

Synthesized as per $\mathbf{6}$ from 2-cyano- $N$-[3-(2-cyanoacetylamino)pentyl]acetamide $\quad(\mathbf{5 d})$ and $\quad 4$ hydroxybenzaldehyde. Afforded a yellow solid, $98 \% \mathrm{mp} 242-244^{\circ} \mathrm{C}$.

${ }^{1} \mathrm{H}$ NMR (DMSO): 8.24 (2H, t $\left.J=5.5 \mathrm{~Hz}\right), 7.99(2 \mathrm{H}, \mathrm{s}) 7.84(4 \mathrm{H}, \mathrm{d} J=8.8 \mathrm{~Hz}), 6.88$ (4H, d $\left.J=8.7 \mathrm{~Hz}\right), 3.18$ $(4 \mathrm{H}$, q $J=6.9 \mathrm{~Hz}), 1.48(4 \mathrm{H}$, quin $J=7.3 \mathrm{~Hz}), 1.28(2 \mathrm{H}$, quin $J=6.8 \mathrm{~Hz})$.

${ }^{13}$ C NMR (DMSO): 162.0, 161.4, 150.1, 132.7, 122.6, 117.2, 116.2, 101.0, 39.5, 28.5, 23.7. 
3-(2-Chlorophenyl)-N-\{5-[3-(2-chlorophenyl)-2-cyanoacryloylamino]pentyl\}-2-cyanoacrylamide (51).

Synthesized as per $\mathbf{6}$ from 2-cyano- $N$-[3-(2-cyanoacetylamino)pentyl]acetamide (5d) and 2-chlorobenzaldehyde. Afforded a yellow solid, $96 \% \mathrm{mp} 164-166^{\circ} \mathrm{C}$.

${ }^{1} \mathrm{H}$ NMR (DMSO): 8.56 (2H, t $\left.J=5.5 \mathrm{~Hz}\right), 8.34(2 \mathrm{H}, \mathrm{s}), 7.98(2 \mathrm{H}, \mathrm{m}), 7.54(6 \mathrm{H}, \mathrm{m}), 3.23(4 \mathrm{H}, \mathrm{q} J=6.1 \mathrm{~Hz}), 1.55$ $(4 \mathrm{H}$, quin $J=7.0 \mathrm{~Hz}), 1.32(2 \mathrm{H}$, quin $J=6.7 \mathrm{~Hz})$.

${ }^{13}$ C NMR (DMSO): 159.9, 146.6, 134.0, 133.1, 130.3, 129.9, 129.6, 127.7, 115.4, 110.5, 39.6, 28.4, 23.7.

3-(4-Chlorophenyl)-N-\{5-[3-(4-chlorophenyl)-2-cyanoacryloylamino]pentyl\}-2-cyanoacrylamide (52).

Synthesized as per $\mathbf{6}$ from 2-cyano- $N$-[3-(2-cyanoacetylamino)pentyl]acetamide (5d) and 4-chlorobenzaldehyde. Afforded a yellow solid, $84 \% \mathrm{mp} 160-162^{\circ} \mathrm{C}$.

${ }^{1} \mathrm{H}$ NMR (DMSO): 8.45 (2H, t $J=5.3 \mathrm{~Hz}$ ), $8.12(2 \mathrm{H}, \mathrm{s}), 7.90$ (4H, d $J=6.9 \mathrm{~Hz}$ ), 7.60 (4H, d $\left.J=8.6 \mathrm{~Hz}\right), 3.20$ (4H, q $J=6.0 \mathrm{~Hz}), 1.51(4 \mathrm{H}$, quin $J=7.1 \mathrm{~Hz}), 1.32(2 \mathrm{H}$, quin $J=6.7 \mathrm{~Hz})$.

${ }^{13}$ C NMR (DMSO): 160.6, 148.8, 136.7, 131.5, 130.7, 129.2, 116.1, 107.0, 39.5, 28.4, 23.6.

2-Cyano- $N$-\{5-[2-cyano-3-(3-methoxyphenyl)acryloylamino]pentyl\}-3-(3-methoxyphenyl)acrylamide (53).

Synthesized as per $\mathbf{6}$ from 2-cyano- $N$-[3-(2-cyanoacetylamino)pentyl]acetamide (5d) and 3-methoxybenzaldehyde. Afforded a yellow solid, $96 \% \mathrm{mp} 124-126^{\circ} \mathrm{C}$.

.${ }^{1} \mathrm{H}$ NMR (DMSO): 8.43 (2H, t $J=5.6 \mathrm{~Hz}$ ), $8.10(2 \mathrm{H}, \mathrm{s}), 7.47(6 \mathrm{H}, \mathrm{m}), 7.13(2 \mathrm{H}, \mathrm{m}), 3.79(6 \mathrm{H}, \mathrm{s}) 3.21(4 \mathrm{H}, \mathrm{q} J=$ $6.7 \mathrm{~Hz}), 1.51(4 \mathrm{H}$, quin $J=7.1 \mathrm{~Hz}), 1.32(2 \mathrm{H}$, quin $J=6.7 \mathrm{~Hz})$.

${ }^{13}$ C NMR (DMSO): 160.7, 159.3, 150.1, 133.1, 130.2, 122.3, 118.0, 116.3, 114.7, 106.7, 55.2, 39.5, 28.4, 23.6.

2-Cyano- $N$-\{5-[2-cyano-3-(4-methoxyphenyl)acryloylamino]pentyl\}-3-(4-methoxyphenyl)acrylamide (54).

Synthesized as per $\mathbf{6}$ from 2-cyano- $N$-[3-(2-cyanoacetylamino)pentyl]acetamide $\quad(\mathbf{5 d})$ and $\quad$ methoxybenzaldehyde. Afforded a yellow solid, $87 \% \mathrm{mp} 132-134^{\circ} \mathrm{C}$.

${ }^{1} \mathrm{H}$ NMR (DMSO): 8.30 (2H, t $\left.J=5.6 \mathrm{~Hz}\right), 8.06(2 \mathrm{H}, \mathrm{s}), 7.94(4 \mathrm{H}, \mathrm{d} J=8.9 \mathrm{~Hz}), 7.08(4 \mathrm{H}, \mathrm{d} J=8.9 \mathrm{~Hz}), 3.83$ (6H, s), $3.20(4 \mathrm{H}$, q $J=6.0 \mathrm{~Hz}), 1.50(4 \mathrm{H}$, quin $J=7.1 \mathrm{~Hz}), 1.30(4 \mathrm{H}$, quin $J=5.6 \mathrm{~Hz})$.

${ }^{13}$ C NMR (DMSO): 162.4, 161.1, 149.7, 132.3, 124.4, 116.9, 114.7, 102.7, 55.5, 39.5, 28.5, 23.6.

2-Cyano-N-\{5-[2-cyano-3-(3,4-dimethoxyphenyl)acryloylamino]pentyl\}-3-(3,4-dimethoxyphenyl)acrylamide (55).

Synthesized as per $\mathbf{6}$ from 2-cyano- $N$-[3-(2-cyanoacetylamino)pentyl]acetamide $\quad(\mathbf{5 d})$ and 3,4dimethoxybenzaldehyde. Afforded a yellow solid, $80 \% \mathrm{mp}>300^{\circ} \mathrm{C}$.

${ }^{1} \mathrm{H}$ NMR (DMSO): 8.28 (2H, t $\left.J=5.5 \mathrm{~Hz}\right), 8.05(2 \mathrm{H}, \mathrm{s}), 7.61(2 \mathrm{H}, \mathrm{dd} J=1.9 \mathrm{~Hz}), 7.54(2 \mathrm{H}, \mathrm{dd} J=8.5 \mathrm{~Hz}, 1.8$ $\mathrm{Hz}), 7.10(2 \mathrm{H}, \mathrm{d} J=8.6 \mathrm{~Hz}), 3.83(6 \mathrm{H}, \mathrm{s}), 3.78(6 \mathrm{H}, \mathrm{s}), 3.20(4 \mathrm{H}, \mathrm{q} J=6.1 \mathrm{~Hz}), 1.51(4 \mathrm{H}$, quin $J=7.1 \mathrm{~Hz}), 1.31$ $(2 \mathrm{H}$, quin $J=6.8 \mathrm{~Hz})$.

${ }^{13}$ C NMR (DMSO): 161.1, 152.3, 150.1, 148.5, 125.2, 124.4, 117.1, 112.2, 11.7, 102.6, 55.7, 55.3, 55.3, 39.4, 28.5, 23.6.

2-Cyano-N-\{5-[2-cyano-3-(3-hydroxy-4-methoxyphenyl)acryloylamino]pentyl\}-3-(3-hydroxy-4methoxyphenyl)acrylamide (56).

Synthesized as per 6 from 2-cyano- $N$-[3-(2-cyanoacetylamino)pentyl]acetamide (5d) and 3-hydroxy-4methoxybenzaldehydeafforded a yellow solid, $92 \% \mathrm{mp} 196-198^{\circ} \mathrm{C}$.

${ }^{1} \mathrm{H}$ NMR (DMSO): 8.27 (2H, t $\left.J=5.5 \mathrm{~Hz}\right), 7.94(2 \mathrm{H}, \mathrm{s}), 7.51(2 \mathrm{H}, \mathrm{d} J=2.2 \mathrm{~Hz}), 7.36$ (2H, dd $J=8.6 \mathrm{~Hz}, 2.2$ $\mathrm{Hz}), 7.05(2 \mathrm{H}, \mathrm{d} J=8.6 \mathrm{~Hz}), 3.84(6 \mathrm{H}, \mathrm{s}), 3.19(2 \mathrm{H}, \mathrm{q} J=6.6 \mathrm{~Hz}), 1.50(2 \mathrm{H}$, quin $J=7.0 \mathrm{~Hz}), 1.29(2 \mathrm{H}$, quin $J=$ $4.7 \mathrm{~Hz})$.

${ }^{13}$ C NMR (DMSO): 161.3, 151.6, 150.0, 146.6, 124.5, 116.9, 115.4, 111.9, 102.3, 55.6, 39.5, 28.5, 23.7. 
2-Cyano- $N$-\{5-[2-cyano-3-(4-carboxyphenyl)acryloylamino]pentyl\}-3-(4-carboxyphenyl)acrylamide (57).

Synthesized as per $\mathbf{6}$ from 2-cyano- $N$-[3-(2-cyanoacetylamino)pentyl]acetamide $\quad(\mathbf{5 d})$ and 4carboxylbenzaldehyde. Afforded a white solid, $74 \% \mathrm{mp} 226-228^{\circ} \mathrm{C}$.

${ }^{1} \mathrm{H}$ NMR (DMSO): 8.52 (2H, br t), 8.18 (2H, s), 8.03 (4H, d $\left.J=8.9 \mathrm{~Hz}\right), 7.98$ (4H,d $\left.J=8.9 \mathrm{~Hz}\right), 3.21$ (4H, q $J=$ $6.4 \mathrm{~Hz}), 1.54(4 \mathrm{H}$, quin $J=6.9 \mathrm{~Hz}), 1.32(2 \mathrm{H}$, quin $J=4.9 \mathrm{~Hz})$.

${ }^{13}$ C NMR (DMSO): 166.6, 160.5, 149.1, 135.5, 134.0, 129.8, 129.7, 115.9, 108.4, 39.5, 28.4, 23.6.

2-Cyano- $N$-[6-(2-cyano-3-phenylacryloylamino)hexyl]-3-phenylacrylamide (58).

Synthesized as per $\mathbf{6}$ from 2-cyano- $N$-[3-(2-cyanoacetylamino)hexyl]acetamide (5e) and benzaldehyde. Afforded a yellow solid, $72 \% \mathrm{mp} 166-168^{\circ} \mathrm{C}$.

${ }^{1} \mathrm{H}$ NMR (DMSO): 8.43 (2H, t $\left.J=5.5 \mathrm{~Hz}\right), 8.14(2 \mathrm{H}, \mathrm{s}), 7.91(4 \mathrm{H}, \mathrm{m}), 7.54(6 \mathrm{H}, \mathrm{m}), 3.21$ (4H, quin $J=6.3 \mathrm{~Hz}$ ), 1.50 (4H, quin $J=6.2 \mathrm{~Hz}), 1.31$ (4H, br quin).

${ }^{13}$ C NMR (DMSO): 160.7, 150.2, 132.1, 131.9, 129.9, 129.1, 116.3, 106.5, 39.6, 28.7, 26.0.

2-Cyano- $N$-\{6-[2-cyano-3-(3-hydroxyphenyl)acryloylamino] hexyl\}-3-(3-hydroxyphenyl)acrylamide (59).

Synthesized as per 6 from 2-cyano- $N$-[3-(2-cyanoacetylamino)hexyl]acetamide (5e) and 3-hydroxybenzaldehyde. Afforded a white solid, $70 \% \mathrm{mp} 234-236^{\circ} \mathrm{C}$.

${ }^{1} \mathrm{H}$ NMR (DMSO): 8.39 (2H, t $J=5.5 \mathrm{~Hz}$ ), 8.02 (2H, s), 7.33 (6H, m), $6.96(2 \mathrm{H}, \mathrm{m}), 3.18$ (4H, q $\left.J=6.1 \mathrm{~Hz}\right), 1.50$ (4H, br quin), 1.30 (4H, br quin).

${ }^{13}$ C NMR (DMSO): 160.8, 157.7, 150.2, 133.0, 130.2, 121.3, 119.5, 116.3, 115.7, 106.1, 39.6, 28.7, 26.0.

2-Cyano-N-\{6-[2-cyano-3-(4-hydroxyphenyl)acryloylamino] hexyl\}-3-(4-hydroxyphenyl)acrylamide (60).

Synthesized as per 6 from 2-cyano- $N$-[3-(2-cyanoacetylamino)hexyl]acetamide (5e) and 4-hydroxybenzaldehyde. Afforded a bright yellow solid, $65 \% \mathrm{mp} 272-276^{\circ} \mathrm{C}$.

${ }^{1} \mathrm{H}$ NMR (DMSO): 8.21 (2H, t $J=5.4 \mathrm{~Hz}$ ), 8.00 (2H, s), 7.84 (4H, d $\left.J=8.7 \mathrm{~Hz}\right), 6.90$ (4H, d $J=8.5 \mathrm{~Hz}$ ), 3.18 (4H, q $J=6.2 \mathrm{~Hz}$ ), 1.48 (4H, br quin), 1.29 (4H, br quin).

${ }^{13}$ C NMR (DMSO): 161.7, 161.3, 150.1, 132.7, 132.7, 122.8, 117.2, 116.1, 101.2, 39.5, 28.8, 26.0.

3-(2-Chlorophenyl)-N-\{6-[3-(2-chlorophenyl)-2-cyanoacryloylamino] hexyl\}-2-cyanoacrylamide (64).

Synthesized as per 6 from 2-cyano- $N$-[3-(2-cyanoacetylamino)hexyl]acetamide (5e) and 2-chlorobenzaldehyde. Afforded a yellow solid, $91 \% \mathrm{mp} 160-162^{\circ} \mathrm{C}$.

${ }^{1} \mathrm{H}$ NMR (DMSO): 8.56 (2H, t $J=5.5 \mathrm{~Hz}$ ), 8.33 (2H, s), 7.98 (2H, m), 7.56 (6H, m), 3.22 (4H, q $\left.J=6.3 \mathrm{~Hz}\right), 1.52$ (4H, quin $J=5.9 \mathrm{~Hz}$ ), 1.32 (4H, br quin).

${ }^{13}$ C NMR (DMSO): 159.9, 146.6, 134.0, 133.1, 130.4, 130.0, 129.6, 127.7, 115.3, 110.6, 39.7, 28.6, 26.0.

3-(4-Chlorophenyl)-N-\{6-[3-(4-chlorophenyl)-2-cyanoacryloylamino] hexyl\}-2-cyanoacrylamide (65).

Synthesized as per 6 from 2-cyano- $N$-[3-(2-cyanoacetylamino)hexyl]acetamide (5e) and 4-chlorobenzaldehyde. Afforded a yellow solid, $72 \% \mathrm{mp} 198-200^{\circ} \mathrm{C}$.

${ }^{1} \mathrm{H}$ NMR (DMSO): 8.45 (2H, t $J=5.3 \mathrm{~Hz}$ ), $8.12(2 \mathrm{H}, \mathrm{s}), 7.92(2 \mathrm{H}, \mathrm{d} J=6.9 \mathrm{~Hz}), 7.62$ (2H, d $\left.J=6.9 \mathrm{~Hz}\right), 3.20$ (4H, quin $J=6.2 \mathrm{~Hz}), 1.50(4 \mathrm{H}$, quin $J=6.0 \mathrm{~Hz}), 1.31$ (4H, br quin).

${ }^{13}$ C NMR (DMSO): 160.5, 148.9, 136.7, 131.5, 130.8, 129.2, 116.0, 107.0, 39.6, 28.6, 26.0.

2-Cyano- $N$-\{6-[2-cyano-3-(3-methoxyphenyl)acryloylamino] hexyl\}-3-(3-methoxyphenyl)acrylamide (66).

Synthesized as per 6 from 2-cyano- $N$-[3-(2-cyanoacetylamino)hexyl]acetamide (5e) and 3-methoxybenzaldehyde. Afforded a yellow solid, $77 \% \mathrm{mp} 136-138^{\circ} \mathrm{C}$.

${ }^{1} \mathrm{H}$ NMR (DMSO): 8.42 (2H, t $\left.J=5.5 \mathrm{~Hz}\right), 8.12(2 \mathrm{H}, \mathrm{s}), 7.46$ (6H, m), 7.14 (2H, m), 3.80 (6H, s), 3.22 (4H, q $J=$ $6.0 \mathrm{~Hz}), 1.51$ (4H, br quin), 1.31 (4H, br quin). 
${ }^{13}$ C NMR (DMSO): 160.7, 159.3, 150.1, 133.1, 130.2, 122.3, 118.0, 116.3, 114.7, 106.7, 55.2, 39.6, 28.7, 26.0.

2-Cyano-N-\{6-[2-cyano-3-(4-methoxyphenyl)acryloylamino] hexyl\}-3-(4-methoxyphenyl)acrylamide (67).

Synthesized as per 6 from 2-cyano- $N$-[3-(2-cyanoacetylamino)hexyl]acetamide (5e) and 4-methoxybenzaldehyde. Afforded a yellow solid, $98 \% \mathrm{mp} 182-184^{\circ} \mathrm{C}$.

${ }^{1} \mathrm{H}$ NMR (DMSO): 8.29 (2H, t $J=5.5 \mathrm{~Hz}$ ), 8.06 (2H, s), 7.93 (2H, d $J=7.2 \mathrm{~Hz}$ ), 7.11 (2H, d $\left.J=7.2 \mathrm{~Hz}\right), 3.84$ (6H, s), 3.17 (4H, q $J=5.7 \mathrm{~Hz}$ ), 1.49 (4H, br quin), 1.30 (4H, br quin).

${ }^{13}$ C NMR (DMSO): 162.4, 161.0, 149.7, 132.23, 124.4, 116.9, 114.7, 102.7, 55.5, 39.5, 28.7, 26.0.

2-Cyano-N-\{6-[2-cyano-3-(3,4-dimethoxyphenyl)acryloylamino] hexyl\}-3-(3,4-dimethoxyphenyl)acrylamide (68).

Synthesized as per $\mathbf{6}$ from 2-cyano- $N$-[3-(2-cyanoacetylamino)hexyl]acetamide $\quad(\mathbf{5 e})$ and 3,4dimethoxybenzaldehyde. Afforded a yellow solid, $76 \% \mathrm{mp}>300^{\circ} \mathrm{C}$.

${ }^{1} \mathrm{H}$ NMR (DMSO): 8.28 (2H, t $\left.J=5.5 \mathrm{~Hz}\right)$ ), $8.05(2 \mathrm{H}, \mathrm{s}), 7.63(2 \mathrm{H}, \mathrm{d} J=2.0 \mathrm{~Hz}), 7.55$ (2H, dd $J=2 \mathrm{~Hz}, 8.3 \mathrm{~Hz}$ ), $7.12(2 \mathrm{H}, \mathrm{d} J=8.3 \mathrm{~Hz}), 3.83(6 \mathrm{H}, \mathrm{s}), 3.78(6 \mathrm{H}, \mathrm{s}), 3.19(4 \mathrm{H}, \mathrm{q} J=6.1 \mathrm{~Hz}), 1.50(4 \mathrm{H}$, quin $J=6.1 \mathrm{~Hz}), 1.30(4 \mathrm{H}, \mathrm{br}$ quin).

${ }^{13}$ C NMR (DMSO): 161.1, 152.3, 150.2, 148.6, 125.2, 124.4, 117.1, 112.2, 111.7, 102.7, 55.7, 55.4, 39.5, 28.7, 26.0

2-Cyano-N-\{6-[2-cyano-3-(3-hydroxy-4-methoxyphenyl)acryloylamino] hexyl\}-3-(3-hydroxy-4methoxyphenyl)acrylamide (69).

Synthesized as per 6 from 2-cyano- $N$-[3-(2-cyanoacetylamino)hexyl]acetamide (5e) and 3-hydroxy-4methoxybenzaldehyde. Afforded a yellow solid, $99 \% \mathrm{mp} 226-228^{\circ} \mathrm{C}$.

${ }^{1} \mathrm{H}$ NMR (DMSO): $8.26(2 \mathrm{H}, \mathrm{t} J=5.6 \mathrm{~Hz}), 7.95(2 \mathrm{H}, \mathrm{s}), 7.52(2 \mathrm{H}, \mathrm{d} J=1.9 \mathrm{~Hz}), 7.36(2 \mathrm{H}, \mathrm{dd} J=8.2 \mathrm{~Hz}, 1.9$ $\mathrm{Hz}$ ), 7.06 (2H, d $J=8.2 \mathrm{~Hz}$ ), 3.84 (6H, s), 3.18 (4H, q $J=5.8 \mathrm{~Hz}$ ), 1.49 (4H, br quin), 1.29 (4H, br quin).

${ }^{13}$ C NMR (DMSO): 161.3, 151.6, 150.1, 146.7, 124.6, 124.5, 116.9, 115.4, 111.9, 102.3, 55.6, 39.5, 28.8, 26.0

2-Cyano- $N$-\{6-[2-cyano-3-(4-carboxyphenyl)acryloylamino] hexyl\}-3-(4-carboxyphenyl)acrylamide (70).

Synthesized as per 6 from 2-cyano- $N$-[3-(2-cyanoacetylamino)hexyl]acetamide (5e) and 4-carboxybenzaldehyde. Afforded a yellow solid, $67 \% \mathrm{mp} 288-292^{\circ} \mathrm{C}$.

${ }^{1} \mathrm{H}$ NMR (DMSO): $8.53(2 \mathrm{H}, \mathrm{t} J=5.1 \mathrm{~Hz}), 8.18(2 \mathrm{H}, \mathrm{s}), 8.07(4 \mathrm{H}, \mathrm{d} J=8.3 \mathrm{~Hz}), 7.98(4 \mathrm{H}, \mathrm{d} J=8.3 \mathrm{~Hz}), 3.20$ (4H, q $J=5.8 \mathrm{~Hz}), 1.51$ (4H, br s), 1.31 (4H, br s).

${ }^{13}$ C NMR (DMSO): 166.6, 160.5, 149.1, 135.5, 134.0, 129.8, 129.7, 116.0, 108.4, 39.5, 28.6, 26.0

2-Cyano-N-[14-(2-cyanoacetylamino)tetradecyl]acetamide (5f)

Synthesized as per 5a from 1,14-diaminotertradecane (3f) and methylcyanoacetate (4). Afforded a white solid, $72 \%, \mathrm{mp} 120^{\circ} \mathrm{C}$.

${ }^{1} \mathrm{H}$ NMR (DMSO): 8.32 (2H, t $\left.J=5.1 \mathrm{~Hz}\right), 3.56$ (4H, s), 2.96 (4H, br q), 1.60 (4H, m), 1.26 (16H, m).

${ }^{13}$ C NMR (DMSO): 162.5, 116.6, 39.1, 31.8, 30.1, 29.8, 27.1.

2-Cyano-N-\{14-[2-cyano-3-(3,4-dihydroxyphenyl)acryloylamino]tetradecyl\}-3-(3,4-dihydroxyphenyl)acrylamide

(71)

Synthesised as per $\mathbf{6}$ from 2-cyano- $N$-[14-(2-cyanoacetylamino)tetradecyl]acetamide $\quad$ (5f) and 3,4dihydroxybenzaldehyde. Afforded a white solid, $75 \%$, mp $218^{\circ} \mathrm{C}$.

${ }^{1} \mathrm{H}$ NMR (DMSO): 8.29 (2H, t $J=5.2 \mathrm{~Hz}$ ), 7.94 (2H, s), 7.43 (2H, d $J=2.2 \mathrm{~Hz}$ ), 7.15 (2H, dd $J=2.2 \mathrm{~Hz}, 8.2 \mathrm{~Hz}$ ), 6.75 (2H, d $J=8.1 \mathrm{~Hz}), 2.96(4 \mathrm{H}, \mathrm{m}), 1.55(\mathrm{~m}), 1.30(16 \mathrm{H}, \mathrm{m})$.

${ }^{13}$ C NMR (DMSO): 163.2, 151.2, 151.0, 146.4, 126.4, 123.1, 117.6, 116.3, 116.1, 100.1, 42.6, 31.7, 30.3, 30.0, 27.4. 
2-Cyano-N-\{14-[2-cyano-3-(3,4,5-trihydroxyphenyl)acryloylamino]tetradecyl\}-3-(3,4,5-

trihydroxyphenyl)acrylamide (72)

Synthesised as per $\mathbf{6}$ from 2-cyano- $N$-[14-(2-cyanoacetylamino)tetradecyl]acetamide (5f) and 3,4,5trihydroxybenzaldehyde. Afforded a white solid, $63 \%, \mathrm{mp} 242^{\circ} \mathrm{C}$.

${ }^{1} \mathrm{H}$ NMR (DMSO): 8.30 (2H, t $J=5.3 \mathrm{~Hz}$ ), 7.75 (2H, s), 6.93 (4H, s), 2.99 (4H, m), 1.59 (4H, m), 1.29 (16H, m).

${ }^{13}$ C NMR (DMSO): 162.4, 150.9, 145.5, 140.1, 121.4, 117.6, 110.1, 99.3, 42.3, 31.3, 30.1, 29.8, 27.1.

2-Cyano-N-\{14-[2-cyano-3-(3,4-dihydroxy-5-methoxyphenyl)acryloylamino]tetradecyl\}-3-(3,4-dihydroxy-5methoxyphenyl)acrylamide (73)

Synthesised as per 6 from 2-cyano- $N$-[14-(2-cyanoacetylamino)tetradecyl]acetamide (5f) and 3,4-dihydroxy-5methoxybenzaldehyde. Afforded an orange solid, $45 \%$, $\mathrm{mp} 233^{\circ} \mathrm{C}$.

${ }^{1} \mathrm{H}$ NMR (DMSO): $8.32(2 \mathrm{H}, J=5.0 \mathrm{~Hz}$ ), $7.92(1 \mathrm{H}, \mathrm{s}), 7.21(2 \mathrm{H}, \mathrm{d} J=2.3 \mathrm{~Hz}), 7.10(2 \mathrm{H}, \mathrm{d} J=2.3 \mathrm{~Hz}), 3.01(4 \mathrm{H}$, $\mathrm{m}), 1.63(4 \mathrm{H}, \mathrm{m}), 1.34(16 \mathrm{H}, \mathrm{m})$.

${ }^{13}$ C NMR (DMSO): 161.1, 150.4, 148.2, 145.3, 139.6, 121.3, 117.2, 111.3, 107.4, 100.9, 42.3, 31.3, 30.2, 30.0, 27.2.

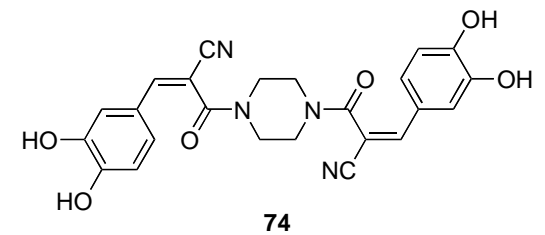

3-[4-(2-Cyanoacetyl)piperazin-1-yl]-3-oxo-propionitrile (5g).

Synthesized as per 5 a from piperazine hydrate (3g) (3 g, $29 \mathrm{mmol})$ and methylcyanoacetate (4). Afforded a white solid, $55 \%$, mp $140^{\circ} \mathrm{C}\left(\right.$ Lit $\left.140^{\circ} \mathrm{C}\right) .{ }^{[1]}$

${ }^{1} \mathrm{H}$ NMR (DMSO): 3.78 (4H, s), 3.56 (8H, m).

${ }^{13}$ C NMR (DMSO): 165.2, 115.1, 42.1, 20.2. (74).

Synthesized as per $\mathbf{9}$ from 3-[4-(2-cyanoacetyl)piperazin-1-yl]-3-oxopropionitrile $\quad(\mathbf{5 f}) \quad$ and $\quad 3,4-$ dihydroxybenzaldehyde. Afforded a yellow solid, $79 \%$, mp $175^{\circ} \mathrm{C}\left(\right.$ Lit $\left.177^{\circ} \mathrm{C}\right) .{ }^{[1]}$

${ }^{1} \mathrm{H}$ NMR (DMSO): 7.90 (2H, s), 7.55 (2H, d, $\left.J=2.0 \mathrm{~Hz}\right), 7.22$ (2H, dd, $\left.J=8.0,2.0 \mathrm{~Hz}\right), 6.82(2 \mathrm{H}, \mathrm{d}, J=8.0 \mathrm{~Hz})$, $3.12(8 \mathrm{H}, \mathrm{m})$.

${ }^{13}$ C NMR (DMSO): 163.5, 150.9, 150.7, 145.6, 145.6, 124.6, 123.4, 117.0, 115.7, 115.6, 99.9, 39.7. 


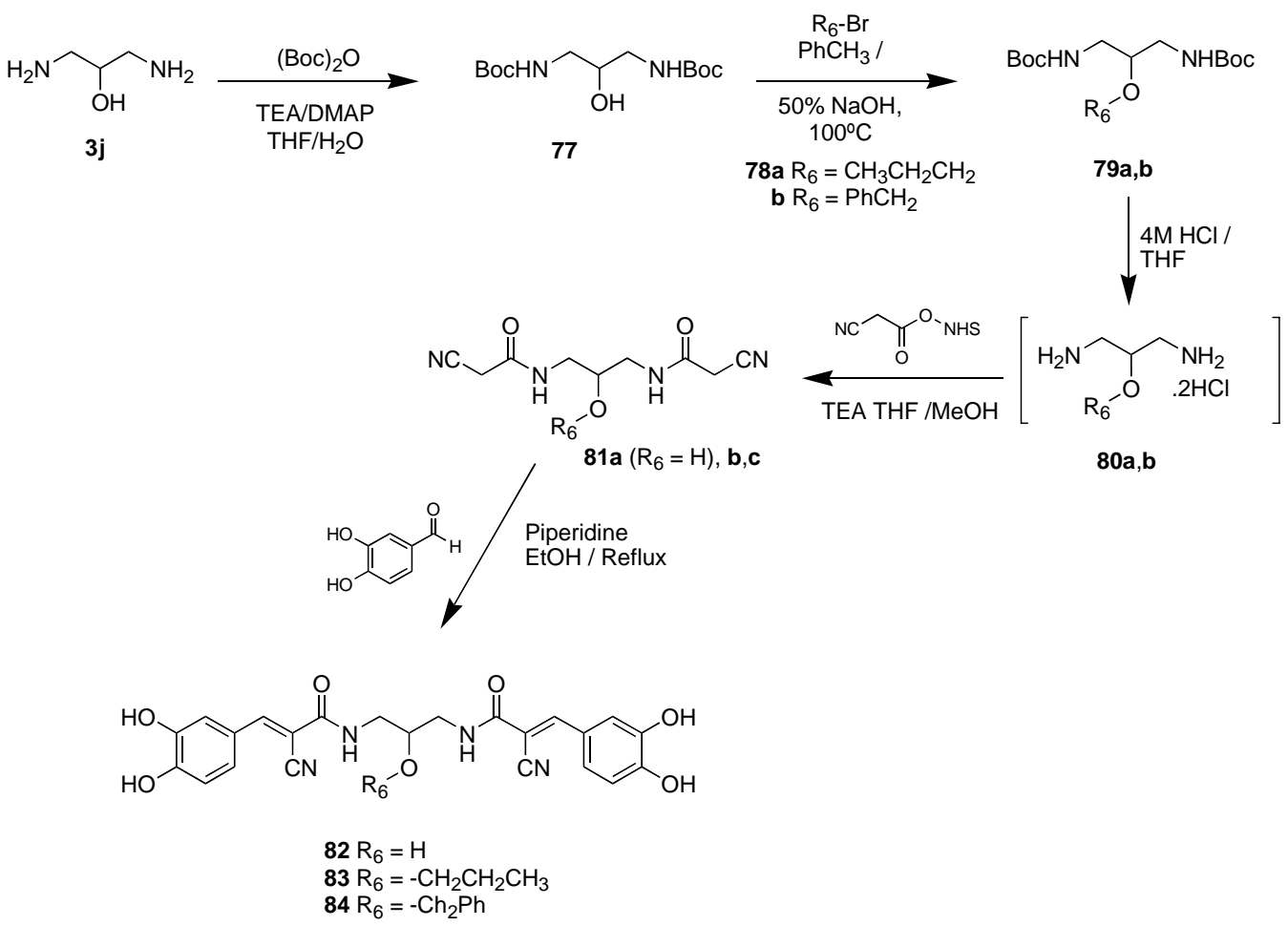

Scheme 2. Introduction of $-\mathrm{OH}(\mathbf{8 2}),-\mathrm{OCH}_{2} \mathrm{CH}_{2} \mathrm{CH}_{3}(\mathbf{8 3})$ and $-\mathrm{OCH}_{2} \mathrm{Ph}(\mathbf{8 4})$.

\section{Di-Boc-1,3-daiminopropane-2-ol (77)}

To a solution of 1,3-diaminopropan-2-ol (3j) (4 g, $44 \mathrm{mmol})$, triethylamine (8.8 g, $88 \mathrm{mmol})$ and DMAP (1.2 g, $10 \mathrm{mmol})$ in $\mathrm{THF} / \mathrm{H}_{2} \mathrm{O}(1: 1150 \mathrm{~mL})$ was added in portions di-tert-butyldicarbonate (22.8 $\left.\mathrm{g}, 10.5 \mathrm{mmol}\right)$. The resulting solution was stirred at room temperature overnight, acidified $(\mathrm{pH} \sim 2)$ with $1 \mathrm{M} \mathrm{HCl}$ and extracted with ethyl acetate $(2 \times 50 \mathrm{~mL})$. The organic extract was dried $\left(\mathrm{MgSO}_{4}\right)$ and the solvent removed in vacuo to yield the desired product as clear oil, which solidified upon standing, $93 \%(11.9 \mathrm{~g})$, mp $120^{\circ} \mathrm{C}$ (lit. $\left.122^{\circ} \mathrm{C}\right){ }^{[2]}$

${ }^{1} \mathrm{H}$ NMR $\left(\mathrm{CDCl}_{3}\right.$ ): 5.3 (br s, 2H), 3.74 (m, 1H), 3.25-3.11 (br m, 4H), 1.42 (s, 18H)

${ }^{13} \mathrm{C} \mathrm{NMR}\left(\mathrm{CDCl}_{3}\right): 155.9,79.7,70.2,41.5,28.1$

\section{2-propoxypropane-di-Boc-1,3-diamine (79a)}

To a stirred solution of di-Boc-1,3-diaiminopropane-2-ol (77) (2 g, $6.5 \mathrm{mmol})$, bromopropane (78a) (19.5 mmol) in toluene $(15 \mathrm{~mL})$ were added aqueous $\mathrm{NaOH} 50 \%(15 \mathrm{~mL})$ and $\mathrm{Bu}_{4} \mathrm{NHSO}_{4}(0.44 \mathrm{~g}, 1.3 \mathrm{mmol})$ at ambient temperature. The resulting solution was stirred for 18 hours at $100^{\circ} \mathrm{C}$, cooled and diluted with EtOAc and brine. The organic phase was dried $\left(\mathrm{MgSO}_{4}\right)$ and concentrated in vacuo. The residue was purified by column chromatography (hexane : EtOAc 7:3). Afforded a white solid, 39\%, 0.83g.

${ }^{1} \mathrm{H} \mathrm{NMR}\left(\mathrm{CDCl}_{3}\right): 5.12$ (br s, 2H), 3.44 (t, 2H, $J=6.7 \mathrm{~Hz}$ ), 3.39-3.07 (m, 5H), 1.54 (m, 2H), 1.42 (s, $\left.18 \mathrm{H}\right), 0.89$ $(\mathrm{t}, 3 \mathrm{H}, J=6.7 \mathrm{~Hz})$

${ }^{13} \mathrm{C} \mathrm{NMR}\left(\mathrm{CDCl}_{3}\right):$ 155.9, 78.7, 76.1, 70.8, 39.9, 27.8, 22.6, 9.9.

\section{2-Benzyloxypropane-di-Boc-1,3-diamine (79b)}

Synthesized as per 79a from di-Boc-1,3-diaiminopropane-2-ol (77) and benzylbromide (78b). Afforded a white solid, $62 \%$.

${ }^{1} \mathrm{H}$ NMR (CDCl 3$): 7.34-7.30$ (m, 5H), 5.04 (br s, 2H), 4.59 (s, 2H), 3.55 (m, 1H), 3.45-3.09 (m, $\left.4 \mathrm{H}\right), 1.43$ (s, $18 \mathrm{H})$.

${ }^{13} \mathrm{C}$ NMR $\left(\mathrm{CDCl}_{3}\right):$ 155.8, 137.5, 127.9, 127.8, 127.2, 78.8, 76.1, 71.0, 39.9, 27.8.

\section{N-[2-Propoxy-3-(2-cyanoacetylamino)propyl]-2-cyanoacetamide (81a)}

2-Propoxypropane-di-Boc-1,3-diamine (79a) $(1.5 \mathrm{mmol})$ was treated with $4 \mathrm{~N} \mathrm{HCl}$ in $\mathrm{THF}(10 \mathrm{~mL})$ for 3 hours at room temperature. The resulting precipitate filtered (80a), washed with $\mathrm{Et}_{2} \mathrm{O}(2 \times 25 \mathrm{~mL})$ and used directly in the 
next step. To a chilled solution of cyanoacetic acid (1.65 mmol) and N-hydroxysuccinimide (NHS) (1.65 mmol) was added dicyclohexyldiimide (DCC) $(1.65 \mathrm{mmol})$. The mixture was stirred at $25^{\circ} \mathrm{C}$ overnight, filtered to remove precipitated dicyclohexyl urea (DHU) and the solvent removed in vacuo. The resulting N-hydroxysuccinyl ester was used directly in the next step. To a stirred solution of the hydrochloride derivatives of $\mathbf{8 0 a}(1.35 \mathrm{mmol})$ and triethylamine $(0.687 \mathrm{~mL}, 5.4 \mathrm{mmol})$ in $\mathrm{MeOH}(10 \mathrm{~mL})$ was added dropwise a solution of cyanoacetic acid NHS ester $(0.491 \mathrm{~g}, 2.7 \mathrm{mmol})$. After the reaction mixture was stirred at $25^{\circ} \mathrm{C}$ overnight, the solvent was removed in vacuo and the crude product recrystallised from $\mathrm{MeOH}, 37 \%, 0.21 \mathrm{~g}$.

${ }^{1} \mathrm{H}$ NMR $\left(\mathrm{CDCl}_{3}\right)$ : 8.26 (br s, 2H), 3.62 (s, 4H), 3.41-3.37 (m, 1H), 3.14-3.09 (m, 2H), 3.01-2.97 (m, 4H), 1.50-1.47 (m 2H), $0.83(\mathrm{t}, 3 \mathrm{H}, J=7.3 \mathrm{~Hz})$.

${ }^{13} \mathrm{C}$ NMR $\left(\mathrm{CDCl}_{3}\right): 162.3,116.1,75.7,70.6,45.4,25.2,22.6,8.7$.

\section{$\mathrm{N}$-[2-Benzyloxy-3-(2-cyanoacetylamino)propyl]-2-cyanoacetamide (81b)}

Synthesized as per 80a from 2-benzyloxypropane-di-Boc-1,3-diamine (79b), Afforded a white solid, 88\%.

${ }^{1} \mathrm{H}$ NMR $\left(\mathrm{CDCl}_{3}\right): 8.43$ (t, 2H, 5.5 Hz), 7.34-7.30 (m, 5H), 4.54 (s, 2H), 3.65 (s, 4H), 3.39 (m, 1H), 3.20-3.05 (m, $4 \mathrm{H})$.

${ }^{13} \mathrm{C}$ NMR $\left(\mathrm{CDCl}_{3}\right): 162.3,138.3,128.0,127.6,127.3,116.0,75.8,70.5,48.5,25.8$

$N$-\{2-Propoxy-3-[2-cyano-3-(3,4-dihydroxyphenyl)acryloylamino]propyl\}-2-cyano-3(3,4dihydroxyphenyl)acrylamide (83)

Synthesized as per 6 from $N$-[2-propoxy-3-(2-cyanoacetylamino)propyl]-2-cyanoacetamide (81a). Afforded a white solid, $26 \%$, mp $194^{\circ} \mathrm{C}$.

${ }^{1} \mathrm{H}$ NMR (DMSO): 8.26 (br s, 2H), 7.93 (s, 2H), 7.52 (br s, 2H), 7.27 (d, 2H, $J=7.9 \mathrm{~Hz}$ ), 6.85 (d, $2 \mathrm{H}, J=8.1$ Hz), 3.67 (m, 1H), 3.46 (m, 2H), 3.36-3.15 (m, 4H), 1.47 (m, 2H), 0.83 (t, 3H, $J=7.3 \mathrm{~Hz})$

${ }^{13}$ C NMR (DMSO): 162.3, 151.1, 150.7, 145.7, 125.3, 123.0, 117.1, 115.8, 115.7, 100.1, 76.7, 70.7, 42.4, 25.2, 10.4

Anal. $\left(\mathrm{C}_{27} \mathrm{H}_{28} \mathrm{~N}_{4} \mathrm{O}_{7} \cdot 0.25 \mathrm{H}_{2} \mathrm{O}\right): \mathrm{C}, \mathrm{H}, \mathrm{N}$.

N-\{2-Benzyloxy-3-[2-cyano-3-(3,4-dihydroxyphenyl)acryloylamino]propyl\}-2-cyano-3(3,4dihydroxyphenyl)acrylamide (84).

Synthesized as per 6 from $N$-[2-benzyloxy-3-(2-cyanoacetylamino)propyl]-2-cyanoacetamide (81b). Afforded a white solid, $72 \%, 258^{\circ} \mathrm{C}$.

${ }^{1} \mathrm{H}$ NMR (DMSO): 8.22 (br s, 2H), 7.93 (s, 2H), 7.53 (br s, 2H), 7.36-7.18 (m, 7H), 6.86 (d, 2H, J = 8.3 Hz), 4.61 (s, 2H), 3.68-3.54 (m, 1H), 3.37-3.16 (m, 4H)

${ }^{13}$ C NMR (DMSO): 161.8, 150.7, 150.6, 145.6, 138.3, 128.0, 127.5, 127.3, 125.2, 123.2, 117.1, 116.0, 115.9, 100.3, 75.8, 70.4, 47.3.

Anal. $\left(\mathrm{C}_{30} \mathrm{H}_{26} \mathrm{~N}_{4} \mathrm{O}_{7} \cdot 0.5 \mathrm{H}_{2} \mathrm{O}\right): \mathrm{C}, \mathrm{H}, \mathrm{N}$. 


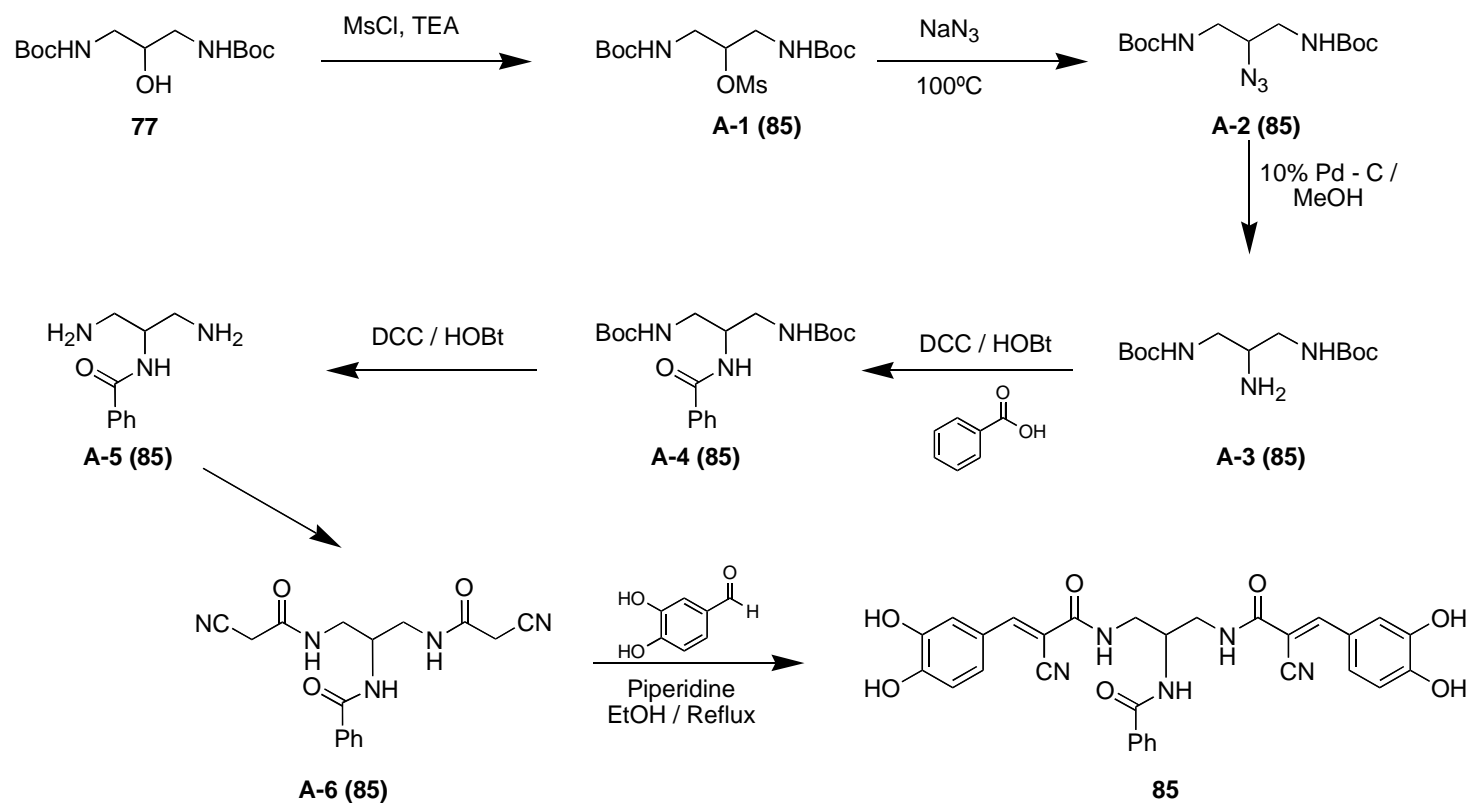

Scheme. Synthesis of benzoyl amide analogue of $6{ }^{1}$

\section{Methanesulfonic acid 2-tert-butoxycarbonylamino-1-(tert-butoxycarbonylaminomethyl)ethyl ester (A-1 (85) $\left.{ }^{1}\right)$}

To a chilled solution of $77(10.8 \mathrm{~g}, 37.4 \mathrm{mmol})$ and triethylamine $(5.8 \mathrm{~g}, 56 \mathrm{mmol})$ in dry $\mathrm{CH}_{2} \mathrm{Cl}_{2}(40 \mathrm{~mL})$ was added methane sulfonyl chloride $(5.6 \mathrm{~g}, 48.6 \mathrm{mmol})$. The resulting solution was stirred at room temperature for $1 \mathrm{hr}$ and water $(25 \mathrm{~mL})$ added cautiously to quench the reaction. The organic layer was separated and washed sequentially with $1 \mathrm{M} \mathrm{HCl}(25 \mathrm{~mL})$, saturated brine $(25 \mathrm{~mL}), 10 \% \mathrm{NaHCO}_{3}(25 \mathrm{~mL})$ and saturated brine $(25 \mathrm{~mL})$. The organic extract was dried $\left(\mathrm{MgSO}_{4}\right)$ and the solvent removed under reduced pressure to yield the desired product as a white solid, $84 \%, 11.5 \mathrm{~g}, \mathrm{mp} 94^{\circ} \mathrm{C}\left(\text { lit. } 93^{\circ} \mathrm{C}\right)^{[4]}$

${ }^{.1} \mathrm{H} \mathrm{NMR}\left(\mathrm{CDCl}_{3}\right): 5.31$ (br s, 2H), 4.72 (m, 1H), 3.45 (m, 4H), 3.08 (s, 3H), 1.42 (s, 18H)

${ }^{13} \mathrm{C}$ NMR $\left(\mathrm{CDCl}_{3}\right)$ : 155.9, 79.7, 78.2, 41.5, 38.2, 28.1.

\section{2-Azidopropane-1,3-di-Boc-diamine (A-2 (85))}

To a solution of methanesulfonic acid 2-tert-butoxycarbonylamino-1-(tert-butoxycarbonylaminomethyl)ethyl ester (A-1 (85)) (10.3 g, $28 \mathrm{mmol})$ in DMF $(50 \mathrm{~mL})$ was added $\mathrm{NaN}_{3}(10.3 \mathrm{~g}, 160 \mathrm{mmol})$ and the mixture was stirred at $100^{\circ} \mathrm{C}$ for 18 hours. The reaction mixture was cooled, poured into water (50 mL) and extracted with EtOAc ( 3 x $50 \mathrm{~mL}$ ). The combined organic extracts were washed with water and brine, dried over $\mathrm{MgSO}_{4}$ and evaporated under reduced pressure to yield the desired product as an orange oil, which solidified upon standing, yield $94 \%$, 8.3g, mp $90^{\circ} \mathrm{C}\left(\right.$ lit. $\left.91^{\circ} \mathrm{C}\right) .{ }^{[4]}$

${ }^{1} \mathrm{H}$ NMR ( $\mathrm{CDCl}_{3}$ ): 5.10 (br s, 2H), 3.71 (m, 1H), 3.31-3.08 (m, 4H), 3.08 (s, 3H), 1.42 (s, 18H).

${ }^{13} \mathrm{C} \mathrm{NMR}\left(\mathrm{CDCl}_{3}\right): \delta 155.9,79.7,60.8,40.3,28.4$.

\section{1,3-di-Boc-1,2,3-triamine (A-3 (85))}

To a solution of 2-azidopropane-1,3-di-Boc-diamine (86) (6 g, $19 \mathrm{mmol})$ in $\mathrm{MeOH}$ (100 mL) was added Pd-C $(10 \% 0.6 \mathrm{~g})$ and the mixture was hydrogenated at $45 \mathrm{psi}$ (18 hours). The reaction mixture was filtered through a pad of celite and evaporated under reduced pressure to yield the desired product as a clear oil, which solidified upon standing, yield $82 \%, 4.5 \mathrm{~g}, \mathrm{mp} 92^{\circ} \mathrm{C}$ (lit. $\left.92^{\circ} \mathrm{C}\right) .{ }^{[4]}$

${ }^{1} \mathrm{H}$ NMR (CDCl $)$ ): 5.10 (br s, 2H), 3.12 (m, 4H), 2.91 (m, 1H), 3.08 (s, 3H), 1.42 (s, 18H)

${ }^{13} \mathrm{C}$ NMR $\left(\mathrm{CDCl}_{3}\right): 155.9,79.7,51.2,44.5,28.3$

\section{(3-tert-Butoxycarbonylamino-2-phenylacetylaminopropyl)carbamic acid tert-butyl ester (A-4 (85))}

\footnotetext{
${ }^{1}$ NOTE: to maintain continuity of compound numbering in the main manuscript a new convention is introduced in this scheme, the number in parentheses refers to the final compound (85) synthesized therein.
} 
To a chilled solution of 1,3 bis-Boc-1,2,3-triamine (A-3 (85)) (0.8 g, $2.77 \mathrm{mmol})$, benzoic acid (0.37 g, $3 \mathrm{mmol})$ and HOBt $(0.41 \mathrm{~g}, 3 \mathrm{mmol})$, in THF (20 mL) was added DCC (0.64 g, $3 \mathrm{mmol})$. The reaction mixture was stirred overnight at $25^{\circ} \mathrm{C}$, filtered and diluted with EtOAc $(25 \mathrm{~mL})$ and saturated brine $(25 \mathrm{~mL})$. The organic layer was then washed sequentially with $1 \mathrm{M} \mathrm{HCl}$, brine $(25 \mathrm{~mL}), 10 \% \mathrm{NaHCO}_{3}(25 \mathrm{~mL})$ and saturated brine $(25 \mathrm{~mL})$ and dried over $\mathrm{MgSO}_{4}$. The solvent was evaporated under reduced pressure to yield the desired product, $78 \%, 0.89 \mathrm{~g}$.

${ }^{1} \mathrm{H}$ NMR $\left(\mathrm{CDCl}_{3}\right): 7.90$ (d, 1H, $\left.J=8.0 \mathrm{~Hz}\right), 7.80$ (d, 2H, $\left.J=6.8 \mathrm{~Hz}\right), 7.49-7.39(\mathrm{~m}, 3 \mathrm{H}), 6.78(\mathrm{br} \mathrm{s}, 2 \mathrm{H}), 4.03$ (p, $1 \mathrm{H}, 6.2 \mathrm{~Hz}), 3.15-3.07$ (m, 4H), $1.36(\mathrm{~s}, 18 \mathrm{H})$

${ }^{13} \mathrm{C} \mathrm{NMR}\left(\mathrm{CDCl}_{3}\right): 166.2,155.9,134.6,130.9,127.9,127.2,77.7,50.3,41.5,28.1$.

\section{$\mathrm{N}$-(2-Amino-1-aminomethylethyl)benzamide (A-5 (85))}

Synthesized as per 80a from (3-tert-butoxycarbonylamino-2-phenylacetylaminopropyl)carbamic acid tert-butyl ester (A-4 (85)). Afforded a white solid, which was used in the next step without purification.

\section{N-\{2-(2-Cyanoacetylamino)-1-[1-(2-cyanoacetylamino)methyl]ethyl\}benzamide (A-6 (85))}

Synthesized as per 81a from $N$-(2-amino-1-aminoethyl)benzamide (A-4 (85)). Afforded a white solid, 28\%.

${ }^{1} \mathrm{H} \mathrm{NMR}\left(\mathrm{CDCl}_{3}\right): 8.26(\mathrm{t}, 2 \mathrm{H}, J=5.6 \mathrm{~Hz}), 8.11(\mathrm{~d}, 1 \mathrm{H}, J=8.2 \mathrm{~Hz}), 7.8$ (dd, 2H, $\left.J=1.5,8.3 \mathrm{~Hz}\right), 7.49$ (m, 3H), 4.11 (m, 1H), 3.60 (s, 4H), $3.28(\mathrm{~m}, 4 \mathrm{H})$

${ }^{13} \mathrm{C}$ NMR $\left(\mathrm{CDCl}_{3}\right): 66.5162 .5$ 134.6, 131.0, 128.0, 127.3, 116.0, 49.2, 40.6, 25.2.

N-(2-[2-Cyano-3-(3,4-dihydroxyphenyl)acryloylamino]-1-\{[2-cyano-3-(3,4-

dihydroxyphenyl)acryloylamino]methyl \}ethyl benzamide (85)

Synthesized as per 6 from $N$-\{2-(2-cyanoacetylamino)-1-[1-(2-cyanoacetylamino)methyl]ethyl $\}$ benzamide (A-6 (85)). Afforded a yellow solid, $28 \%, \mathrm{mp} 244^{\circ} \mathrm{C}$.

${ }^{1} \mathrm{H}$ NMR (DMSO): 8.19 (m, 3H), 7.86 (s, 2H), 7.8 (d, 2H, $J=7$ Hz), 7.46 (m, 5H), 7.20 (d, 2H, $\left.J=6.8 \mathrm{~Hz}\right), 6.72$ (d, $2 \mathrm{H}, J=8 \mathrm{~Hz}), 4.27(\mathrm{~m}, 1 \mathrm{H}), 3.38(\mathrm{~m}, 4 \mathrm{H})$

${ }^{13}$ C NMR (DMSO): $\delta$ 166.5, 162.6, 154.1, 150.6, 146.2, 134.7, 131.0, 128.0, 127.3, 126.4, 116.0, 115.7, 114.5, 97.7, 49.6, 40.3

Anal. $\left(\mathrm{C}_{30} \mathrm{H}_{25} \mathrm{~N}_{5} \mathrm{O}_{7}\right)$ : C, H, N.

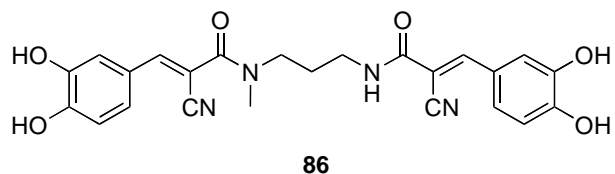

\section{2-Cyano- $N$-[3-(2-cyanoacetyl)-methylamino)propyl]acetamide (5k}

Synthesized as per 5a from $N^{\prime}$-methyl-1,3-propane diamine (3k) and methylcyanoacetate (4) (2.2 g, $22 \mathrm{mmol}$ ). Afforded a white solid, $55 \%, \mathrm{mp} 102^{\circ} \mathrm{C}$.

${ }^{1} \mathrm{H}$ NMR (DMSO): 8.20 (1H, t, $J=5.4 \mathrm{~Hz}$ ), 3.50 (4H, s), 3.25 (4H, br s), 2.92 (3H, s), 1.62 (2H, quin, $J=6.0$ $\mathrm{Hz})$.

${ }^{13}$ C NMR (DMSO): 165.3, 162.1, 115.0, 46.2, 39.7, 30.2, 22.1, 20.2

Anal. $\left(\mathrm{C}_{25} \mathrm{H}_{24} \mathrm{~N}_{4} \mathrm{O}_{6} \cdot 2 \mathrm{H}_{2} \mathrm{O}\right) \mathrm{C}, \mathrm{H}, \mathrm{N}$.

2-Cyano- $N$-\{3-[2-cyano-3-(3,4-dihydroxyphenyl)acryloylamino]propyl\}-3-(3,4-dihydroxyphenyl)- $N$ methylacrylamide (86)

Synthesized as per 6 from 2-cyano- $N$-[3-(2-cyanoacetyl)methylamino)propyl]acetamide (5k) and 3,4dihydroxybenzaldehyde. Afforded a yellow solid, $85 \%, \mathrm{mp} 96^{\circ} \mathrm{C}$.

${ }^{1} \mathrm{H}$ NMR (DMSO): 8.20 (1H, t, J = $\left.5.3 \mathrm{~Hz}\right), 7.90(2 \mathrm{H}, \mathrm{s}), 7.57(2 \mathrm{H}, \mathrm{d}, J=2.0 \mathrm{~Hz}), 7.23(2 \mathrm{H}, \mathrm{dd}, J=8.0,2.0 \mathrm{~Hz})$, $6.84(2 \mathrm{H}, \mathrm{d}, J=8.0 \mathrm{~Hz}$ ), 3.22 (4H, br s), 2.99 (3H, s), 1.72 (2H, quin, $J=6.0 \mathrm{~Hz}$ ).

${ }^{13} \mathrm{C}$ NMR (DMSO): 165.3, 162.1, 153.1, 151.2, 146.6, 144.6, 125.3, 124.4, 116.3, 115.3, 115.1, 99.9, 46.2, 39.7, $30.2,22.1,20.2$ 


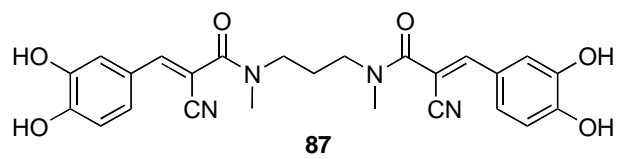

2-Cyano-N-[3-(2-cyanoacetyl)methylamino)propyl]-N-methylacetamide (5I)

Synthesized as per 5a from $N^{\prime}, N^{\prime}$-dimethylpropane-1,3-diamine (3l) and methylcyanoacetate (4). Afforded a white solid, $55 \%, 84^{\circ} \mathrm{C}$.

${ }^{1} \mathrm{H}$ NMR (DMSO): 3.48 (4H, s), 3.20 (4H, t, $\left.J=6.0 \mathrm{~Hz}\right), 2.92(6 \mathrm{H}, \mathrm{s}), 1.81(2 \mathrm{H}$, quin, $J=6.0 \mathrm{~Hz})$.

${ }^{13}$ C NMR (DMSO): 164.9, 115.0, 45.3, 32.1, 28.3

2-Cyano-N-(3-\{[2-cyano-3-(3,4-dihydroxyphenyl)acryloyl]methylamino\}propyl)-3-(3,4-dihydroxyphenyl)- $N$ methylacrylamide (87)

Synthesized as per 6 from 2-cyano- $N$-[3-(2-cyanoacetyl)methylamino)propyl]-N-methylacetamide (5l) and 3,4dihydroxybenzaldehyde. Afforded a yellow-green solid, $79 \%, \mathrm{mp} 224^{\circ} \mathrm{C}$.

${ }^{1} \mathrm{H}$ NMR (DMSO): 7.90 (2H, s), 7.55 (2H, d, $\left.J=2.0 \mathrm{~Hz}\right), 7.22$ (2H, dd, $\left.J=8.0,2.0 \mathrm{~Hz}\right), 6.82(2 \mathrm{H}, \mathrm{d}, J=8.0 \mathrm{~Hz}$ ), $3.22(4 \mathrm{H}, \mathrm{t}, J=6.1 \mathrm{~Hz}), 2.89(6 \mathrm{H}, \mathrm{s}), 1.82(2 \mathrm{H}$, quin, $J=6.0 \mathrm{~Hz})$.

${ }^{13}$ C NMR (DMSO): 163.5, 152.1, 150.2, 145.6, 145.6, 124.6, 123.4, 117.0, 115.7, 115.6, 99.9, 45.3, 32.1, 28.3

Anal. $\left(\mathrm{C}_{24} \mathrm{H}_{22} \mathrm{~N}_{4} \mathrm{O}_{6} \cdot 2 \cdot 5 \mathrm{H}_{2} \mathrm{O}\right) \mathrm{C}, \mathrm{H}, \mathrm{N}$.

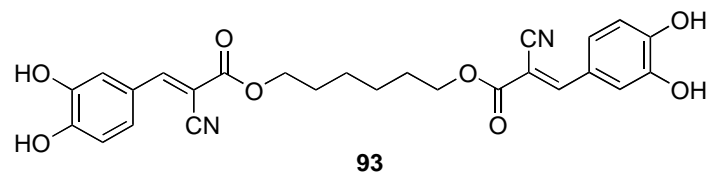

Cyanoacetic acid 6-(2-cyanoacetoxy)hexyl ester (89d)

Synthesized as per 89a from 1,6-hexanediol (88d), methylcyanoacetate (4) and titanium (IV) butoxide. Afforded a white solid, $57 \%, \mathrm{mp} 70^{\circ} \mathrm{C}\left(\right.$ lit. $\left.69^{\circ} \mathrm{C}\right) .^{[3]}$

${ }^{1} \mathrm{H}$ NMR (DMSO): 4.13 (4H, t $\left.J=6.5 \mathrm{~Hz}\right), 3.97(4 \mathrm{H}, \mathrm{s}), 1.59(4 \mathrm{H}, \mathrm{t} \mathrm{J}=6.5 \mathrm{~Hz}), 1.33$ (4H, quin $\left.J=3.75\right)$

${ }^{13}$ C NMR (DMSO): 164.2, 114.9, 65.6, 27.6, 24.9, 24.3

2-Cyano-3-(3,4-dihydroxyphenyl)acrylic acid 2-[2-cyano-3-(3,4-dihydroxyphenyl)acryloyloxy-hexyl ester (93).

Synthesized as per 9 from cyanoacetic acid 2-(2-cyanoacetoxy)hexyl ester (89c) and 3,4-dihydroxybenzaldehyde. Afforded a yellow solid, $64 \%, \mathrm{mp} 262^{\circ} \mathrm{C}$.

${ }^{1} \mathrm{H}$ NMR (DMSO): 8.10 (2H, t br), 7.62 (2H, d $J=2.0 \mathrm{~Hz}$ ), 7.35 (2H, dd $\left.J=8.5 \mathrm{~Hz}, 2.0 \mathrm{~Hz}\right), 6.85$ (2H, d $J=8.5$ $\mathrm{Hz}), 4.28$ (4H, t br), $1.79(4 \mathrm{H}$, quin br) $1.79(4 \mathrm{H}$, quin br) $1.38(4 \mathrm{H}$, quin br)

${ }^{13}$ C NMR (DMSO): 162.3, 154.2, 151.9, 146.0, 127.4, 122.2, 116.3, 116.0, 115.8, 95.7, 64.8, 24.6, 22.3

Anal. $\left(\mathrm{C}_{26} \mathrm{H}_{24} \mathrm{~N}_{4} \mathrm{O}_{8}\right): \mathrm{C}, \mathrm{H}, \mathrm{N}$.

\section{3,4-diacetoxycaffeic acid (95)}

To a cold solution of caffeic acid (94) 3.5g $(19.4 \mathrm{mmol})$ and DMAP $(0.06 \mathrm{~g}, 0.5 \mathrm{mmol})$ in pyridine $(9 \mathrm{~mL})$ was added acetic anhydride $(4.55 \mathrm{~mL}, 48.4 \mathrm{mmol})$. The mixture was stirred at room temperature for 1 hour and poured over crushed ice. The solution was acidified $(\mathrm{pH}<2)$ and extracted with EtOAc $(3 \times 25 \mathrm{~mL})$. The combined extracts were dried over $\mathrm{MgSO}_{4}$ and concentrated in vacuo.

${ }^{1} \mathrm{H}$ NMR (DMSO): 12.83 (br s, $1 \mathrm{H}$ ), 7.47 (d, 1H, $J=16 \mathrm{~Hz}$ ), 7.33 (dd, $1 \mathrm{H}, J=2.1,8.3 \mathrm{~Hz}$ ), 7.29 (d, $1 \mathrm{H}, J=2$ $\mathrm{Hz}$ ), 7.12 (d, $1 \mathrm{H} J=8.4 \mathrm{~Hz}), 6.29$ (d, 1H, $J=16 \mathrm{~Hz}), 2.2$ (s, 6H)

${ }^{13}$ C NMR (DMSO): 170.9, 167.3, 165.3, 145.9, 144.3, 142.2, 131.2, 126.8, 123.7, 123.2, 118.9, 20.0

\section{3,4-Diacetoxycaffeic acid chloride (96)}

To a stirred solution of 3,4-diacetoxycaffeic acid (95) (3 g, $11.25 \mathrm{mmol}$ ) in dry $\mathrm{CHCl}_{3}(20 \mathrm{~mL}$ ) containing 2 drops of DMF was added $\mathrm{SOCl}_{2}(1.06 \mathrm{ml}, 30 \mathrm{mmol})$. The resulting solution was refluxed for $4 \mathrm{~h}$, allowed to cool and the solvent and excess $\mathrm{SOCl}_{2}$ removed in vacuo. The acid chloride was used directly in the next step.

${ }^{1} \mathrm{H}$ NMR (DMSO): 7.77 (d, 1H, $J=15.5 \mathrm{~Hz}$ ), 7.46 (dd, 1H, $J=2,8.3 \mathrm{~Hz}$ ), 7.26 (d, 1H, $J=1.9 \mathrm{~Hz}$ ), 7.28 (d, $1 \mathrm{H}$, $J=8.3 \mathrm{~Hz}), 6.59$ (d, $1 \mathrm{H}, J=15.5 \mathrm{~Hz}), 2.31(\mathrm{~s}, 6 \mathrm{H})$

${ }^{13}$ C NMR (DMSO): 161.4, 167.3, 165.3, 147.9, 144.3, 142.2, 131.2, 126.8, 123.7, 123.2, 122.9, 20.0 


\section{3-(3,4-Diacetoxyphenyl)- $\mathrm{N}$-\{2-[3-(3,4-acetoxyphenyl)acryloylamino]ethyl\}acrylamide (97a)}

To a stirred solution of the appropriate 1,2-ethylenediamine (3a) $(0.9 \mathrm{~g}, 1.5 \mathrm{mmol})$ and triethylamine $(0.3 \mathrm{~g}, 3$ $\mathrm{mmol})$ in dry $\mathrm{CH}_{2} \mathrm{Cl}_{2}(10 \mathrm{~mL})$ was added dropwise a solution of 3,4-diacetoxycaffeic acid chloride (96) $(1 \mathrm{~g}, 3.7$ $\mathrm{mmol})$ in dry $\mathrm{CH}_{2} \mathrm{Cl}_{2}(10 \mathrm{~mL})$. After the reaction mixture was stirred at $25^{\circ} \mathrm{C}$ overnight, EtOAc $(25 \mathrm{~mL})$ and saturated brine $(25 \mathrm{~mL})$ were added. The organic layer was then washed sequentially with $1 \mathrm{M} \mathrm{HCl}(25 \mathrm{~mL})$, saturated brine $(25 \mathrm{~mL}), 10 \% \mathrm{NaHCO}_{3}(25 \mathrm{~mL})$ and saturated brine $(25 \mathrm{~mL})$ and dried over $\mathrm{MgSO}_{4}$. The solvent was evaporated in vacuo and the crude product recrystallised from EtOAc/Hexane. Afforded a white solid, 62\%, $0.83 \mathrm{~g}, \mathrm{mp} 241^{\circ} \mathrm{C}$.

${ }^{1} \mathrm{H}$ NMR (DMSO): 8.24 (br s, 2H), 7.48 (dd, 2H, $J=2,8.4 \mathrm{~Hz}$ ), 7.46 (d, 2H, $J=1.9 \mathrm{~Hz}$ ), 7.41 (d, 2H, $J=15.8$ $\mathrm{Hz}$ ), 7.29 (d, 2H, $J=8.2 \mathrm{~Hz}$ ), 6.59 (d, 2H, $J=15.8 \mathrm{~Hz}), 3.30$ (m, 4H), 2.27 (s, 12H)

${ }^{13}$ C NMR (DMSO): 168.1, 164.8, 142.5, 142.2, 136.9, 133.7, 125.8, 124.1, 123.1, 122.2, 38.6, 20.2

3-(3,4-Diacetoxyphenyl)-N-\{2-[3-(3,4-acetoxy-phenyl)acryloylamino]propyl\}acrylamide (97b) Synthesized as per 97a from 1,3-diaminopropane (3b). Afforded an orange solid, 67\%.

${ }^{1} \mathrm{H}$ NMR (DMSO): 8.14 (t, 2H, $J=5.6 \mathrm{~Hz}$ ), 7.49 (dd, 2H, $J=2,8.4 \mathrm{~Hz}$ ), 7.47 (d, 2H, $J=1.9 \mathrm{~Hz}$ ), 7.38 (d, 2H, $J=$ $15.8 \mathrm{~Hz}), 7.26$ (d, 2H, $J=8.2 \mathrm{~Hz}), 6.59(\mathrm{~d}, 2 \mathrm{H}, J=15.8 \mathrm{~Hz}), 3.22(\mathrm{~m}, 4 \mathrm{H}), 2.26(\mathrm{~s}, 12 \mathrm{H}), 1.64(\mathrm{~m}, 2 \mathrm{H})$

${ }^{13}$ C NMR (DMSO): 168.1, 168.0, 164.6, 142.5, 142.2, 136.8, 133.7, 125.7, 124.0, 123.3, 122.2, 36.6, 29.2, 20.2

3-(3,4-Diacetoxyphenyl)-N-\{2-[3-(3,4-acetoxyphenyl)acryloylamino]butyl\}acrylamide (97c)

Synthesized as per $\mathbf{9 7 a}$ from 1,4-diaminobutane (3c). Afforded a white solid, 59\%, mp $189^{\circ} \mathrm{C}$.

${ }^{1} \mathrm{H}$ NMR (DMSO): 8.12 (br s, 2H), 7.47 (m, 4H), 7.38 (d, 2H, $J=15.8 \mathrm{~Hz}$ ), 7.28 (d, 2H, $J=8.1 \mathrm{~Hz}$ ), 6.61 (d, 2H, $J=15.8 \mathrm{~Hz}), 3.18(\mathrm{~m}, 4 \mathrm{H}), 2.25$ (s, $12 \mathrm{H}), 1.47(\mathrm{~m}, 4 \mathrm{H})$

${ }^{13}$ C NMR (DMSO): 168.1, 168.0, 164.5, 142.5, 142.2, 136.8, 133.7, 125.7, 124.0, 123.3, 122.0, 38.3, 26.6, 20.5

3-(3,4-Diacetoxyphenyl)-N-\{2-[3-(3,4-acetoxyphenyl)acryloylamino]hexyl\}acrylamide (97d)

Synthesized as per 97a from 1,6-diaminoheptane (3d). Afforded a white solid, 81\%, mp $168^{\circ} \mathrm{C}$.

${ }^{1} \mathrm{H}$ NMR (DMSO): 8.08 (t, 2H, $\left.J=5.5 \mathrm{~Hz}\right), 7.48(\mathrm{~m}, 4 \mathrm{H}), 7.37$ (d, 2H, $\left.J=15.8 \mathrm{~Hz}\right), 7.28(\mathrm{~d}, 2 \mathrm{H}, J=8.1 \mathrm{~Hz})$, 6.59 (d, 2H, $J=15.8 \mathrm{~Hz}$ ), 3.16 (m, 4H), 2.26 (s, 12H), 1.44 (m, 4H), 1.30 (m, 4H)

${ }^{13}$ C NMR (DMSO): 168.1, 168.0, 164.4, 142.5, 142.2, 136.6, 133.8, 125.7, 124.0, 123.4, 122.1, 38.5, 29.0, 26.0, 20.2

3-(3,4-Diacetoxyphenyl)-N-\{2-[3-(3,4-acetoxyphenyl)acryloylamino]dodecyl\}acrylamide (97e)

Synthesized as per $\mathbf{9 7}$ a from 1,12-diaminododecane (3e). Afforded a white solid, $58 \%$, mp $144^{\circ} \mathrm{C}$.

${ }^{1} \mathrm{H}$ NMR (DMSO): 8.1 (br s, 2H), 7.47 (m, 4H), 7.38 (d, 2H, $\left.J=15.8 \mathrm{~Hz}\right), 7.26$ (d, 2H, $\left.J=8.1 \mathrm{~Hz}\right), 6.57$ (d, 2H, $J=15.8 \mathrm{~Hz}$ ), 3.16 (m, 4H), 2.26 (s, 12H), 1.40 (m, 4H), 1.22 (m, 16H)

${ }^{13}$ C NMR (DMSO): 168.1, 168.0, 164.4, 142.5, 142.2, 136.6, 133.8, 125.7, 124.0, 123.4, 122.1, 38.5, 29.1, 28.9, 28.7, 26.4, 20.2

3-(3,4-Dihydroxyphenyl)- $N$-\{2-[3-(3,4-dihydroxyphenyl)acryloylamino] ethyl\}acrylamide (98).

A solution of 3-(3,4-diacetoxyphenyl)- $N$-\{2-[3-(3,4-acetoxyphenyl)acryloylamino]ethyl $\}$ acrylamide 97a (0.71 g, $1.3 \mathrm{mmol})$ in acetone $(25 \mathrm{~mL})$ was treated at $50^{\circ} \mathrm{C}$ for 3 hours with $3 \mathrm{M} \mathrm{HCl}$. After being cooled, the reaction mixture was diluted with EtOAc $(25 \mathrm{~mL})$ and saturated brine $(25 \mathrm{~mL})$. The organic phase was dried over $\mathrm{MgSO}_{4}$, evaporated under reduced pressure and the crude product recrystallised from $\mathrm{MeOH}$. Afforded a white solid, 82\%, $0.42 \mathrm{~g}, \mathrm{mp}>250^{\circ} \mathrm{C}$ (dec.).

${ }^{1} \mathrm{H}$ NMR (DMSO): 9.2 (br s, 4H), 8.08 (br s, 2H), 7.23 (d, 2H, $J=15.7 \mathrm{~Hz}$ ), 6.92 (s, 2H), 6.82 (d, 2H, $J=7.7$ $\mathrm{Hz}), 6.72$ (d, 2H, $J=7.8 \mathrm{~Hz}$ ), 6.29 (d, 2H, $J=15.8 \mathrm{~Hz}), 3.25(\mathrm{~m}, 4 \mathrm{H})$

${ }^{13}$ C NMR (DMSO): 165.6, 147.2, 145.4, 139.1, 126.2, 120.3, 118.3, 115.7, 113.7, 38.6

Anal. $\left(\mathrm{C}_{20} \mathrm{H}_{20} \mathrm{~N}_{2} \mathrm{O}_{8} \cdot 0.5 \mathrm{H}_{2} \mathrm{O}\right)$ : C, $\mathrm{H}, \mathrm{N}$.

3-(3,4-Dihydroxyphenyl)- $N$-\{2-[3-(3,4-dihydroxyphenyl)acryloylamino]propyl\}acrylamide (99).

Synthesized as per 98 from 3-(3,4-diacetoxyphenyl)- $N$-\{2-[3-(3,4-acetoxyphenyl)acryloylamino]propyl\}acrylamide (97b). Afforded a yellow solid, $86 \%, \mathrm{mp}>250^{\circ} \mathrm{C}$ (dec.). 
${ }^{1} \mathrm{H}$ NMR (DMSO): 9.2 (br s, 4H), 7.98 (t, 2H, $J=5.6 \mathrm{~Hz}$ ), 7.22 (d, 2H, $J=15.7 \mathrm{~Hz}$ ), 6.92 (s, 2H), 6.82 (d, 2H, $J$ $=7.7 \mathrm{~Hz}), 6.72(\mathrm{~d}, 2 \mathrm{H}, J=7.8 \mathrm{~Hz}), 6.29(\mathrm{~d}, 2 \mathrm{H}, J=15.8 \mathrm{~Hz}), 3.18(\mathrm{q}, 4 \mathrm{H}, J=6 \mathrm{~Hz}), 1.62(\mathrm{p}, 2 \mathrm{H}, J=6.8 \mathrm{~Hz})$.

${ }^{13} \mathrm{C}$ NMR (DMSO): 165.6, 147.2, 145.4, 138.9, 126.3, 120.3, 118.4, 115.6, 113.7, 36.4, 27.3.

Anal. $\left(\mathrm{C}_{21} \mathrm{H}_{22} \mathrm{~N}_{2} \mathrm{O}_{8} \cdot \mathrm{H}_{2} \mathrm{O}\right)$ : C, $\mathrm{H}, \mathrm{N}$.

3-(3,4-Dihydroxyphenyl)-N-\{2-[3-(3,4-dihydroxyphenyl)acryloylamino]butyl\}acrylamide (100).

Synthesized as per 98 from 3-(3,4-diacetoxyphenyl)- $N$-\{2-[3-(3,4-acetoxyphenyl)acryloylamino]butyl $\}$ acrylamide (97c). Afforded a yellow solid, $86 \%, \mathrm{mp}>250^{\circ} \mathrm{C}$ (dec.).

${ }^{1} \mathrm{H}$ NMR (DMSO): 9.2 (br s, 4H), 7.96 (t, 2H, $J=5.5 \mathrm{~Hz}$ ), 7.20 (d, 2H, $J=15.7 \mathrm{~Hz}$ ), 6.91 (d, 2H, $J=1.8 \mathrm{~Hz}$ ), $6.82(\mathrm{dd}, 2 \mathrm{H}, J=1.7,8.1 \mathrm{~Hz}), 6.72(\mathrm{~d}, 2 \mathrm{H}, J=8.1 \mathrm{~Hz}), 6.29(\mathrm{~d}, 2 \mathrm{H}, J=15.8 \mathrm{~Hz}), 3.18(\mathrm{~m}, 4 \mathrm{H}), 1.45(\mathrm{~m}, 2 \mathrm{H})$

${ }^{13}$ C NMR (DMSO): 165.2, 147.1, 145.4, 138.8, 126.3, 120.2, 118.5, 115.6, 113.7, 38.2, 26.7

Anal. $\left(\mathrm{C}_{22} \mathrm{H}_{24} \mathrm{~N}_{2} \mathrm{O}_{8} \cdot 1.25 \mathrm{H}_{2} \mathrm{O}\right)$ : C, $\mathrm{H}, \mathrm{N}$.

3-(3,4-Dihydroxyphenyl)-N-\{2-[3-(3,4-dihydroxyphenyl)acryloylamino] hexyl\}acrylamide (101).

Synthesized as per 98 from 3-(3,4-diacetoxyphenyl)- $N$ - $\{2$-[3-(3,4-acetoxyphenyl)acryloylamino]hexyl $\}$ acrylamide (97d). Afforded a yellow solid, $62 \%, \mathrm{mp} 218^{\circ} \mathrm{C}$.

${ }^{1} \mathrm{H}$ NMR (DMSO): 7.92 (t, 2H, $J=5.2 \mathrm{~Hz}$ ), 7.19 (d, 2H, $J=15.7 \mathrm{~Hz}$ ), 6.91 (s, 2H), 6.82 (d, 2H, $\left.J=8.3 \mathrm{~Hz}\right), 6.71$ (d, $2 \mathrm{H}, J=8.2 \mathrm{~Hz}), 6.29$ (d, 2H, $J=15.8 \mathrm{~Hz}), 3.18(\mathrm{~m}, 4 \mathrm{H}), 1.43(\mathrm{~m}, 4 \mathrm{H}), 1.29(\mathrm{~m}, 4 \mathrm{H})$

${ }^{13} \mathrm{C}$ NMR (DMSO): 165.2, 147.1, 145.4, 138.8, 126.3, 120.2, 118.5, 115.6, 113.7, 38.2, 29.1, 26.1

Anal. $\left(\mathrm{C}_{24} \mathrm{H}_{28} \mathrm{~N}_{2} \mathrm{O}_{8} \cdot \mathrm{H}_{2} \mathrm{O}\right)$ : C, $\mathrm{H}, \mathrm{N}$.

3-(3,4-Dihydroxyphenyl)-N-\{2-[3-(3,4-dihydroxyphenyl)acryloylamino]dodecyl\}acrylamide (102).

Synthesized as per $\mathbf{9 8}$ from 3-(3,4-diacetoxyphenyl)- $N$ - $\{2$-[3-(3,4acetoxyphenyl)acryloylamino]dodecyl \}acrylamide (97e). Afforded a yellow solid, $82 \%, \mathrm{mp} 220^{\circ} \mathrm{C}$.

${ }^{1} \mathrm{H}$ NMR (DMSO): 9.3 (br s, 4H), 7.90 (br s, 2H), 7.19 (d, 2H, $J=15.6 \mathrm{~Hz}$ ), 6.91 (s, 2H), 6.80 (d, 2H, $J=8.1$ $\mathrm{Hz}), 6.71(\mathrm{~d}, 2 \mathrm{H}, J=8.1 \mathrm{~Hz}), 6.29$ (d, 2H, $J=15.8 \mathrm{~Hz}), 3.11(\mathrm{~m}, 4 \mathrm{H}), 1.40(\mathrm{~m}, 4 \mathrm{H}), 1.22(\mathrm{~m}, 16 \mathrm{H})$

${ }^{13}$ C NMR (DMSO): 165.2, 147.1, 145.4, 138.8, 126.4, 120.2, 118.6, 115.6, 113.7, 38.5, 29.1, 28.1, 28.6, 26.4

Anal. $\left(\mathrm{C}_{30} \mathrm{H}_{40} \mathrm{~N}_{2} \mathrm{O}_{8} \cdot 0.75 \mathrm{H}_{2} \mathrm{O}\right): \mathrm{C}, \mathrm{H}, \mathrm{N}$. 


\section{Library based on Compound 2}

TABLE S1: Effect of analogues of 2 (monomeric, asymmetric) on dynamin I GTPase activity.

\begin{tabular}{|c|c|c|c|c|c|c|c|}
\hline Compound & $\mathbf{R}^{1}$ & $\mathbf{R}^{2}$ & $\mathbf{R}^{3}$ & $\mathbf{R}^{4}$ & $\mathbf{R}^{5}$ & $\mathbf{R}^{6}$ & $\mathrm{IC}_{50} \mu \mathrm{M}$ \\
\hline S8 & $\mathrm{H}$ & $\mathrm{H}$ & $\mathrm{H}$ & $\mathrm{H}$ & $\mathrm{H}$ & $\mathrm{CH}_{2} \mathrm{CH}_{3}$ & - \\
\hline S9 & $\mathrm{H}$ & $\mathrm{OH}$ & $\mathrm{H}$ & $\mathrm{H}$ & $\mathrm{H}$ & $\mathrm{CH}_{2} \mathrm{CH}_{3}$ & - \\
\hline S10 & $\mathrm{H}$ & $\mathrm{H}$ & $\mathrm{OH}$ & $\mathrm{H}$ & $\mathrm{H}$ & $\mathrm{CH}_{2} \mathrm{CH}_{3}$ & - \\
\hline S11 & $\mathrm{H}$ & $\mathrm{H}$ & $\mathrm{OH}$ & $\mathrm{OH}$ & $\mathrm{H}$ & $\mathrm{CH}_{2} \mathrm{CH}_{3}$ & - \\
\hline S12 & $\mathrm{Cl}$ & $\mathrm{H}$ & $\mathrm{H}$ & $\mathrm{H}$ & $\mathrm{H}$ & $\mathrm{CH}_{2} \mathrm{CH}_{3}$ & - \\
\hline S13 & $\mathrm{H}$ & $\mathrm{Br}$ & $\mathrm{H}$ & $\mathrm{H}$ & $\mathrm{H}$ & $\mathrm{CH}_{2} \mathrm{CH}_{3}$ & - \\
\hline S14 & $\mathrm{H}$ & $\mathrm{H}$ & $\mathrm{Cl}$ & $\mathrm{H}$ & $\mathrm{H}$ & $\mathrm{CH}_{2} \mathrm{CH}_{3}$ & - \\
\hline S15 & $\mathrm{H}$ & $\mathrm{OMe}$ & $\mathrm{H}$ & $\mathrm{H}$ & $\mathrm{H}$ & $\mathrm{CH}_{2} \mathrm{CH}_{3}$ & \\
\hline S16 & $\mathrm{H}$ & $\mathrm{H}$ & OMe & $\mathrm{H}$ & $\mathrm{H}$ & $\mathrm{CH}_{2} \mathrm{CH}_{3}$ & - \\
\hline S17 & $\mathrm{H}$ & $\mathrm{H}$ & $\mathrm{OMe}$ & OMe & $\mathrm{H}$ & $\mathrm{CH}_{2} \mathrm{CH}_{3}$ & - \\
\hline S18 & $\mathrm{H}$ & $\mathrm{H}$ & OMe & $\mathrm{OH}$ & $\mathrm{H}$ & $\mathrm{CH}_{2} \mathrm{CH}_{3}$ & - \\
\hline S19 & $\mathrm{H}$ & $\mathrm{NO}_{2}$ & $\mathrm{H}$ & $\mathrm{H}$ & $\mathrm{H}$ & $\mathrm{CH}_{2} \mathrm{CH}_{3}$ & - \\
\hline S20 & $\mathrm{H}$ & $\mathrm{H}$ & $\mathrm{NO}_{2}$ & $\mathrm{H}$ & $\mathrm{H}$ & $\mathrm{CH}_{2} \mathrm{CH}_{3}$ & - \\
\hline S21 & $\mathrm{H}$ & $\mathrm{H}$ & $\mathrm{COOH}$ & $\mathrm{H}$ & $\mathrm{H}$ & $\mathrm{CH}_{2} \mathrm{CH}_{3}$ & - \\
\hline $\mathrm{S} 22$ & $\mathrm{H}$ & $\mathrm{H}$ & $\mathrm{N}\left(\mathrm{CH}_{3}\right)_{2}$ & $\mathrm{H}$ & $\mathrm{H}$ & $\mathrm{CH}_{2} \mathrm{CH}_{3}$ & - \\
\hline $\mathrm{S} 23$ & $\mathrm{H}$ & $\mathrm{H}$ & $\mathrm{H}$ & $\mathrm{H}$ & $\mathrm{H}$ & $\left(\mathrm{CH}_{2}\right)_{4} \mathrm{CH}_{3}$ & - \\
\hline S24 & $\mathrm{H}$ & $\mathrm{OH}$ & $\mathrm{H}$ & $\mathrm{H}$ & $\mathrm{H}$ & $\left(\mathrm{CH}_{2}\right)_{4} \mathrm{CH}_{3}$ & - \\
\hline S25 & $\mathrm{H}$ & $\mathrm{H}$ & $\mathrm{OH}$ & $\mathrm{H}$ & $\mathrm{H}$ & $\left(\mathrm{CH}_{2}\right)_{4} \mathrm{CH}_{3}$ & - \\
\hline S26 & $\mathrm{H}$ & $\mathrm{H}$ & $\mathrm{OH}$ & $\mathrm{OH}$ & $\mathrm{H}$ & $\left(\mathrm{CH}_{2}\right)_{4} \mathrm{CH}_{3}$ & - \\
\hline S27 & $\mathrm{Cl}$ & $\mathrm{H}$ & $\mathrm{H}$ & $\mathrm{H}$ & $\mathrm{H}$ & $\left(\mathrm{CH}_{2}\right)_{4} \mathrm{CH}_{3}$ & - \\
\hline S28 & $\mathrm{H}$ & $\mathrm{Br}$ & $\mathrm{H}$ & $\mathrm{H}$ & $\mathrm{H}$ & $\left(\mathrm{CH}_{2}\right)_{4} \mathrm{CH}_{3}$ & - \\
\hline S29 & $\mathrm{H}$ & $\mathrm{H}$ & $\mathrm{Cl}$ & $\mathrm{H}$ & $\mathrm{H}$ & $\left(\mathrm{CH}_{2}\right)_{4} \mathrm{CH}_{3}$ & - \\
\hline S30 & $\mathrm{H}$ & $\mathrm{OMe}$ & $\mathrm{H}$ & $\mathrm{H}$ & $\mathrm{H}$ & $\left(\mathrm{CH}_{2}\right)_{4} \mathrm{CH}_{3}$ & - \\
\hline S31 & $\mathrm{H}$ & $\mathrm{H}$ & $\mathrm{OMe}$ & $\mathrm{H}$ & $\mathrm{H}$ & $\left(\mathrm{CH}_{2}\right)_{4} \mathrm{CH}_{3}$ & - \\
\hline S32 & $\mathrm{H}$ & $\mathrm{H}$ & $\mathrm{OMe}$ & $\mathrm{OMe}$ & $\mathrm{H}$ & $\left(\mathrm{CH}_{2}\right)_{4} \mathrm{CH}_{3}$ & - \\
\hline S33 & $\mathrm{H}$ & $\mathrm{H}$ & OMe & $\mathrm{OH}$ & $\mathrm{H}$ & $\left(\mathrm{CH}_{2}\right)_{4} \mathrm{CH}_{3}$ & - \\
\hline S34 & $\mathrm{H}$ & $\mathrm{NO}_{2}$ & $\mathrm{H}$ & $\mathrm{H}$ & $\mathrm{H}$ & $\left(\mathrm{CH}_{2}\right)_{4} \mathrm{CH}_{3}$ & - \\
\hline S35 & $\mathrm{H}$ & $\mathrm{H}$ & $\mathrm{NO}_{2}$ & $\mathrm{H}$ & $\mathrm{H}$ & $\left(\mathrm{CH}_{2}\right)_{4} \mathrm{CH}_{3}$ & - \\
\hline S36 & $\mathrm{H}$ & $\mathrm{H}$ & $\mathrm{COOH}$ & $\mathrm{H}$ & $\mathrm{H}$ & $\left(\mathrm{CH}_{2}\right)_{4} \mathrm{CH}_{3}$ & - \\
\hline S37 & $\mathrm{H}$ & $\mathrm{H}$ & $\mathrm{N}\left(\mathrm{CH}_{3}\right)_{2}$ & $\mathrm{H}$ & $\mathrm{H}$ & $\left(\mathrm{CH}_{2}\right)_{4} \mathrm{CH}_{3}$ & - \\
\hline S38 & $\mathrm{H}$ & $\mathrm{H}$ & $\mathrm{H}$ & $\mathrm{H}$ & $\mathrm{H}$ & & - \\
\hline S39 & $\mathrm{H}$ & $\mathrm{OH}$ & $\mathrm{H}$ & $\mathrm{H}$ & $\mathrm{H}$ & & - \\
\hline S40 & $\mathrm{H}$ & $\mathrm{H}$ & $\mathrm{OH}$ & $\mathrm{H}$ & $\mathrm{H}$ & & - \\
\hline S41 & $\mathrm{H}$ & $\mathrm{H}$ & $\mathrm{OH}$ & $\mathrm{OH}$ & $\mathrm{H}$ & & - \\
\hline
\end{tabular}




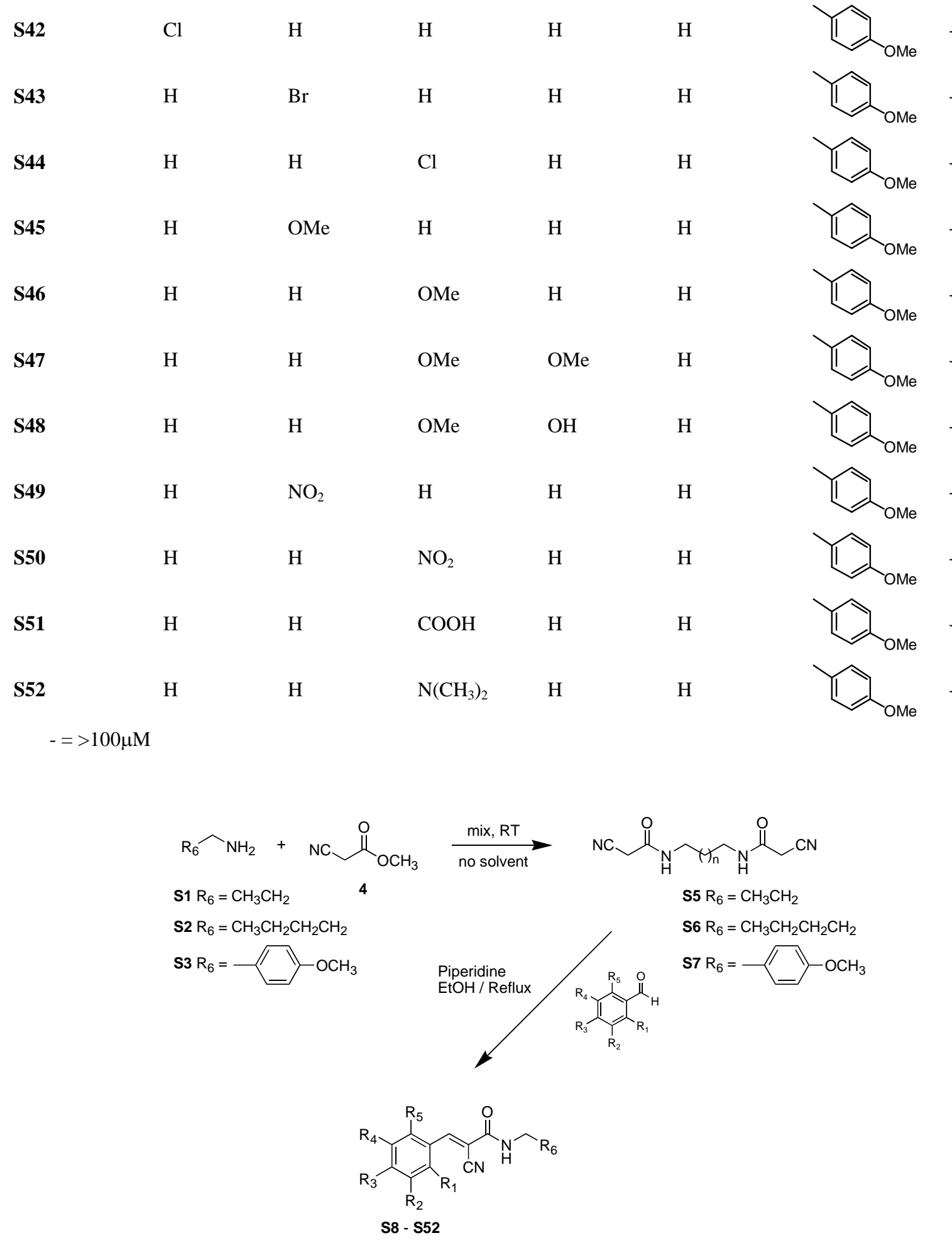

Scheme. Synthesis of 1 analogues.

2-Cyano-N-propylacetamide (S5)

Methylcyanoacetate 4 (3 g, $30 \mathrm{mmol}$ ) and propylamine $\mathbf{S 1}$ (1.78 g, $30 \mathrm{mmol}$ ) were stirred at room temperature (12h). The resulting white solid was recrystallised from ether to give a white solid, $3.2 \mathrm{~g}(84 \%)$.

${ }^{1} \mathrm{H}$ NMR (DMSO): 8.27 (1H, s br), 3.65 (2H, s), 3.13 (2H, q), 1.50 (2H, quin), 0.95 (2H, t)

${ }^{13} \mathrm{C}$ NMR (DMSO): 161.8, 116.1, 40.8, 25.1, 21.9, 11.2. 


\section{2-cyano-3-phenyl-N-propylacrylamide (S8).}

2-cyano- $N$-propylacetamide $\mathbf{S} 5$ ( $0.2 \mathrm{~g}, 1.59 \mathrm{mmol})$, benzaldehyde $(0.17 \mathrm{~g}, 1.59 \mathrm{mmol}), 3$ drops of piperidine and ethanol $(10 \mathrm{~mL})$ were refluxed for 2 hours. Cooling, filtering and washing with cold ether $(10 \mathrm{~mL})$ gave a yellow solid, $0.14 \mathrm{~g}(41.6 \%)$.

${ }^{1} \mathrm{H}$ NMR (DMSO): 8.46 (H, s), 8.02 (1H, d), 7.94 (1H, t), 7.57 (1H, t), $3.17(2 \mathrm{H}, \mathrm{t}), 1.49-1.56(2 \mathrm{H}, \mathrm{m}), 0.88$ (3H, t).

${ }^{13}$ C NMR (DMSO): 160.9, 160.8, 132.1, 131.9, 129.9, 129.1, 116.3, 106.5, 40.3, 22.3, 11.2.

2-cyano-3-(3-hydroxyphenyl)-N-propylacrylamide (S9).

Synthesized as per S8 from 2-cyano- $N$-propylacetamide S5 and 3-hydroxybenzaldehyde. Afforded a white solid, $49 \%$.

${ }^{1} \mathrm{H}$ NMR (DMSO): 8.03 (1H, s), 7.36 (1H, t), 7.33 (1H, d), 6.9 (1H, s), 6.95 (1H, d), 3.18 (2H, t), 1.47-1.55 (2H, $\mathrm{m}), 0.88(3 \mathrm{H}, \mathrm{t})$.

${ }^{13}$ C NMR (DMSO): 165.0, 161.8, 154.4, 137.1, 134.3, 125.4, 123.6, 120.4, 119.8, 110.2, 45.5, 26.2, 15.4.

2-cyano-3-(4-hydroxyphenyl)-N-propylacrylamide (S10).

Synthesized as per S8 from 2-cyano- $N$-propylacetamide S5 and 4-hydroxybenzaldehyde. Afforded a yellow solid, 30\%.

${ }^{1} \mathrm{H}$ NMR (DMSO): 8.22 (1H, s), 7.85 (1H, d), 6.91 (1H, d), 3.16 (2H, t), 1.48-1.55 (2H, m), 0.88 (3H, t).

${ }^{13}$ C NMR (DMSO): 162.7, 161.5, 150.1, 132.8, 132.8, 117.3, 116.3, 100.5, 41.3, 22.1, 11.2.

2-cyano-3-(3,4-dihydroxyphenyl)-N-propylacrylamide (S11).

Synthesized as per S8 from 2-cyano- $N$-propylacetamide $\mathbf{S} 5$ and 3,4-dihydroxybenzaldehyde. Afforded a yellow solid, $53 \%$.

${ }^{1} \mathrm{H}$ NMR (DMSO): 8.24 (1H, s), 7.28 (1H, d), 7.25 (1H, d), 6.87 (1H, s), $3.16(2 \mathrm{H}, \mathrm{t}), 1.48-1.56$ (2H, m), 0.85 $(3 \mathrm{H}, \mathrm{t})$.

${ }^{13}$ C NMR (DMSO): 161.5, 150.6, 150.3, 145.6, 125.0, 123.2, 117.2, 115.9, 115.8, 100.8, 41.3, 22.1, 11.2.

2-cyano-3-(2-chlorophenyl)- $N$-propylacrylamide (S12).

Synthesized as per S8 from 2-cyano- $N$-propylacetamide S5 and 2-chlorobenzaldehyde. Afforded an off-white solid, $42 \%$.

${ }^{1} \mathrm{H}$ NMR (DMSO): 8.36 (1H, s), $8.01(1 \mathrm{H}, \mathrm{d}), 7.98(1 \mathrm{H}, \mathrm{d}), 7.60$ 1(H, t), $7.51(\mathrm{H}, \mathrm{t}), 3.19$ (2H, t), 1.49-1.54 (2H, $\mathrm{m}), 0.86(3 \mathrm{H}, \mathrm{t})$.

${ }^{13}$ C NMR (DMSO): 159.9, 146.6, 134.0, 133.0, 130.3, 129.9, 129.5, 127.6, 115.4, 110.5, 41.4, 22.0, 11.1.

2-cyano-3-(3-bromophenyl)-N-propylacrylamide (S13).

Synthesized as per S8 from 2-cyano- $N$-propyl-acetamide S5 and 3-bromobenzaldehyde. Afforded a white solid, $46 \%$.

${ }^{1} \mathrm{H}$ NMR (DMSO): 8.15 (1H, s), 7.98 (1H, s), 7.94 (1H, d), 7.67 (1H, d), $7.11(1 \mathrm{H}, \mathrm{t}), 3.17$ (2H, t), 1.48-1.55 (2H, $\mathrm{m}), 0.86(3 \mathrm{H}, \mathrm{t})$.

${ }^{13}$ C NMR (DMSO): 162.6, 151.7, 137.0, 132.6, 132.4, 130.7, 127.3, 125.6, 116.6, 102.0, 41.2, 22.1, 11.2.

2-cyano-3-(4-chlorophenyl)-N-propylacrylamide (S14).

Synthesized as per $\mathbf{S 8}$ from 2-cyano- $N$-propylacetamide $\mathbf{S} 5$ and 4-chlorobenzaldehyde. Afforded a yellow solid, $25 \%$.

${ }^{1} \mathrm{H}$ NMR (DMSO): 8.14 (1H, s), 7.93-7.96 (1H, d), 7.62-7.65 (1H, d), 3.15-3.21 (2H, t), 1.49-1.53 (2H, m), 0.85$0.90(3 \mathrm{H}, \mathrm{t})$.

${ }^{13}$ C NMR (DMSO): 160.6, 148.9, 136.7, 131.5, 130.7, 116.1, 107.1, 41.4, 22.0, 11.2.

2-cyano-3-(3-methoxyphenyl)-N-propylacrylamide (S15). 
Synthesized as per S8 from 2-cyano- $N$-propylacetamide S5 and 3-methoxybenzaldehyde. Afforded an off-white solid, $27 \%$.

${ }^{1} \mathrm{H}$ NMR (DMSO): 8.12 (1H, s), $7.51(1 \mathrm{H}, \mathrm{t}), 7.43(1 \mathrm{H}, \mathrm{s}), 7.17(1 \mathrm{H}, \mathrm{d}), 7.15(1 \mathrm{H}, \mathrm{d}), 3.81(3 \mathrm{H}, \mathrm{s}), 3.17(2 \mathrm{H}, \mathrm{t})$, 1.49-1.55 (2H, m), $0.87(3 \mathrm{H}, \mathrm{t})$.

${ }^{13}$ C NMR (DMSO): 160.7, 159.3, 150.1, 133.1, 130.2, 122.3, 118.0, 116.3, 114.7, 106.7, 55.2, 41.4, 22.0, 11.2.

2-cyano-3-(4-methoxyphenyl)-N-propylacrylamide (S16).

Synthesized as per S8 from 2-cyano- $N$-propylacetamide S5 and 4-methoxybenzaldehyde. Afforded a white solid, $45 \%$.

${ }^{1} \mathrm{H}$ NMR (DMSO): 8.07 (1H, s), 7.94 (1H, d), 7.10 (1H, d), 3.40 (3H, s), $3.16(2 \mathrm{H}, \mathrm{t}), 1.47-1.55$ (2H, m), 0.86 $(3 \mathrm{H}, \mathrm{t})$.

${ }^{13}$ C NMR (DMSO): 162.9, 160.4, 148.5, 134.5, 134.2, 122.1, 115.9, 102.7, 41.2, 39.4, 22.0, 11.2.

2-cyano-3-(3,4-dimethoxyphenyl)-N-propylacrylamide (S17).

Synthesized as per S8 from 2-cyano- $N$-propylacetamide S5 and 3,4-dimethoxybenzaldehyde. Afforded an offwhite solid, $49 \%$.

${ }^{1} \mathrm{H}$ NMR (DMSO): 8.06 (1H, s), 7.64 (1H, s), 7.56 (1H, d), 7.11 (1H, d), 3.85 (3H, s), 3.80 (3H, s), 3.17 (2H, t), 1.48-1.55 (2H, m), 0.8 (3H, t).

${ }^{13}$ C NMR (DMSO): 161.1, 152.3, 150.1, 148.6, 125.3, 124.4, 117.0, 112.1, 111.6, 102.6, 55.6, 41.3, 22.1, 11.2.

2-cyano-3-(3-hydroxy-4-methoxyphenyl)- N-propylacrylamide (S18).

Synthesized as per S8 from 2-cyano- $N$-propylacetamide S5 and 3-hydroxy-4-methoxybenzaldehyde. Afforded a yellow solid, 65\%.

${ }^{1} \mathrm{H}$ NMR (DMSO): 7.97 (1H, s), 7.40 (1H, d), 7.37 (1H, d), 7.09 (1H, s), $3.86(3 \mathrm{H}, \mathrm{s}), 3.16$ (2H, t), 1.47-1.54 (2H, $\mathrm{m}), 0.86(3 \mathrm{H}, \mathrm{t})$.

${ }^{13}$ C NMR (DMSO): 161.3, 151.6, 150.1, 146.7, 135.8, 124.5, 116.9, 115.4, 111.9, 102.3, 55.6, 41.3, 22.1, 11.2.

2-cyano-3-(3-nitrophenyl)-N-propylacrylamide (S19).

Synthesized as per S8 from 2-cyano- $N$-propylacetamide S5 and 3-nitrobenzaldehyde. Afforded a yellow solid, $61 \%$.

${ }^{1} \mathrm{H}$ NMR (DMSO): 8.36 (1H, s), 8.32 (1H, s), $8.11(1 \mathrm{H}, \mathrm{d}), 7.67(1 \mathrm{H}, \mathrm{d}), 7.12(1 \mathrm{H}, \mathrm{t}), 3.18(2 \mathrm{H}, \mathrm{t}), 1.48-1.53(2 \mathrm{H}$, $\mathrm{m}), 0.87(3 \mathrm{H}, \mathrm{t})$.

${ }^{13}$ C NMR (DMSO): 160.2, 156.4, 147.9, 135.7, 133.4, 130.7, 126.0, 124.0, 115.6, 109.2, 41.4, 22.1, 11.2.

2-cyano-3-(4-nitrophenyl)-N-propylacrylamide (S20).

Synthesized as per S8 from 2-cyano- $N$-propylacetamide S5 and 4-nitrobenzaldehyde. Afforded a white solid, $80 \%$.

${ }^{1} \mathrm{H}$ NMR (DMSO): 8.10 (1H, s), 7.98 (1H, d), 7.69 (1H, d), 3.18 (2H, t), 1.48-1.54 (2H, m), 0.87 (3H, t).

${ }^{13}$ C NMR (DMSO): 161.8, 154.4, 148.2, 145.7, 128.8, 126.4, 116.6, 101.6, 41.3, 22.1, 11.2

2-cyano-3-(4-carboxyphenyl)-N-propylacrylamide (S21).

Synthesized as per S8 from 2-cyano- $N$-propylacetamide S5 and 4-carboxybenzaldehyde. Afforded a white solid, $58 \%)$.

${ }^{1} \mathrm{H}$ NMR (DMSO): 8.19 (1H, s), 8.04 (1H, d), 7.95 (1H, d), 3.18 (2H, t), 1.49-1.56 (2H, m), 0.87 (3H, t).

${ }^{13}$ C NMR (DMSO): 167.8, 160.7, 150.0, 138.3, 134.0, 129.5, 128.8, 116.2, 107.4, 41.4, 22.0, 11.2.

2-cyano-3-(4-dimethylaminophenyl)- $N$-propylacrylamide (S22).

Synthesized as per S8 from 2-cyano- $N$-propylacetamide S5 and 4-(dimethylamino)benzaldehyde. Afforded a bright orange solid, $63 \%$.

${ }^{1} \mathrm{H}$ NMR (DMSO): 7.93 (1H, s), 7.84 (1H, d), 6.80 (1H, d), 3.36 (3H, s), 3.18 (2H, t), 3.04 (3H, s), 1.46-1.54 (2H, $\mathrm{m}), 0.86(3 \mathrm{H}, \mathrm{t})$.

${ }^{13}$ C NMR (DMSO): 161.9, 152.8, 150.0, 132.5, 118.7, 118.1, 111.5, 97.1, 43.1, 41.2, 22.2, 11.2. 


\section{2-Cyano- $N$-hexylacetamide (S6)}

Synthesised as per $\mathbf{S} 5$ from methylcyanoacetate $\mathbf{4}$ and hexylamine S2. Afforded a white solid, 88\%.

${ }^{1} \mathrm{H}$ NMR (DMSO): 8.25 (1H, s), 3.70 (2H, s), 3.15 (2H, q), 1.50 (2H, quin), 1.35 (8H, m), 0.95 (3H, t)

${ }^{13} \mathrm{C}$ NMR (DMSO): 161.8, 116.1, 38.9, 30.8, 28.6, 25.8, 25.1, 21.9, 13.8

2-cyano-N-hexyl-3-phenylacrylamide (S23).

Synthesized as per $\mathbf{S 8}$ from 2-cyano- $N$-hexylacetamide $\mathbf{S 6}$ and benzaldehyde. Afforded a yellow solid, 37\%.

${ }^{1} \mathrm{H}$ NMR (DMSO): $8.16(1 \mathrm{H}, \mathrm{s}), 7.93(1 \mathrm{H}, \mathrm{d}), 7.57(1 \mathrm{H}, \mathrm{t}), 7.54(1 \mathrm{H}, \mathrm{t}), 3.20(2 \mathrm{H}, \mathrm{t}), 1.47-1.51(2 \mathrm{H}, \mathrm{m}), 1.33-1.38$ (2H, m), 1.25-1.29 (2H, m), $0.85(3 \mathrm{H}, \mathrm{t})$.

${ }^{13}$ C NMR (DMSO): 160.6, 150.2, 132.1, 129.9, 129.1, 129.1, 116.3, 106.4, 39.6, 30.9, 28.7, 26.0, 22.0, 13.8.

2-cyano-N-hexyl-3-(3-hydroxyphenyl)acrylamide (S24).

Synthesized as per $\mathbf{S 8}$ from 2-cyano- $N$-hexylacetamide $\mathbf{S 6}$ and 3-hydroxybenzaldehyde. Afforded a white solid, $57 \%$.

${ }^{1} \mathrm{H}$ NMR (DMSO): $8.04(1 \mathrm{H}, \mathrm{s}), 7.36(1 \mathrm{H}, \mathrm{d}), 7.33(1 \mathrm{H}, \mathrm{t}), 6.99(1 \mathrm{H}, \mathrm{s}), 6.97(1 \mathrm{H}, \mathrm{d}), 3.18(2 \mathrm{H}, \mathrm{t}), 1.46-1.51(2 \mathrm{H}$, m), 1.33-1.38 (2H, m), 1.26-1.29 (2H, m), $0.85(3 \mathrm{H}, \mathrm{t})$.

${ }^{13}$ C NMR (DMSO): 160.8, 157.7, 150.2, 133.0, 130.1, 121.3, 119.5, 116.3, 115.7, 106.01, 42.0, 30.9, 28.7, 26.0, 21.9, 13.8

2-cyano-N-hexyl-3-(4-hydroxyphenyl)acrylamide (S25).

Synthesized as per $\mathbf{S 8}$ from 2-cyano- $N$-hexylacetamide $\mathbf{S 6}$ and 4-hydroxybenzaldehyde. Afforded a yellow solid, $50 \%$.

${ }^{1} \mathrm{H}$ NMR (DMSO): $8.02(1 \mathrm{H}, \mathrm{s}), 7.86(1 \mathrm{H}, \mathrm{d}), 6.92(1 \mathrm{H}, \mathrm{d}), 3.18(2 \mathrm{H}, \mathrm{t}), 1.45-1.49(2 \mathrm{H}, \mathrm{m}), 1.35(2 \mathrm{H}, \mathrm{t}), 1.25-$ $1.29(2 \mathrm{H}, \mathrm{m}), 0.85(3 \mathrm{H}, \mathrm{t})$.

${ }^{13}$ C NMR (DMSO): 164.8, 161.6, 150.1, 132.7, 122.8, 117.2, 116.1, 101.2, 39.6, 30.9, 28.8, 26.0, 21.9, 13.8.

2-cyano-N-hexyl-3-(3,4-dihydroxyphenyl)acrylamide (S26).

Synthesized as per $\mathbf{S 8}$ from 2-cyano- $N$-hexylacetamide $\mathbf{S 6}$ and 3,4-dihydroxybenzaldehyde. Afforded a yellow solid, $57 \%$.

${ }^{1} \mathrm{H}$ NMR (DMSO): $7.92(1 \mathrm{H}, \mathrm{s}), 7.54$ (1H, s), 7.27 (1H, d), $6.87(1 \mathrm{H}, \mathrm{d}), 3.17(2 \mathrm{H}, \mathrm{t}), 1.45-1.47(2 \mathrm{H}, \mathrm{m}), 1.33-$ $1.38(2 \mathrm{H}, \mathrm{m}), 1.25-1.28(2 \mathrm{H}, \mathrm{m}), 0.85(3 \mathrm{H}, \mathrm{t})$.

${ }^{13}$ C NMR (DMSO): 166.5, 150.6, 150.3, 145.6, 125.0 123.2, 117.1, 115.9, 115.8, 100.8, 39.3, 30.9, 28.8, 26.0, 21.9, 13.8 .

2-cyano-N-hexyl-3-(2-chlorophenyl)acrylamide (S27).

Synthesized as per S8 from 2-cyano- $N$-hexylacetamide $\mathbf{S 6}$ and 2-chlorobenzaldehyde. Afforded a white solid, $13 \%$

${ }^{1} \mathrm{H}$ NMR (DMSO): $8.34(1 \mathrm{H}, \mathrm{s}), 8.01(1 \mathrm{H}, \mathrm{d}), 7.79(1 \mathrm{H}, \mathrm{d}), 7.64(1 \mathrm{H}, \mathrm{t}), 7.57(1 \mathrm{H}, \mathrm{t}), 3.21(2 \mathrm{H}, \mathrm{t}), 1.46-1.50(2 \mathrm{H}$, $\mathrm{m}), 1.33-1.38(2 \mathrm{H}, \mathrm{m}), 1.25-1.28(2 \mathrm{H}, \mathrm{m}), 0.87(3 \mathrm{H}, \mathrm{t})$.

${ }^{13}$ C NMR (DMSO): 161.8, 159.8, 133.9, 133.1, 130.4, 130.0, 129.6, 127.7, 115.4, 110.6, 38.6, 30.8, 28.6, 25.9, 21.9, 13.8 .

2-cyano-N-hexyl-3-(3-bromophenyl)acrylamide (S28).

Synthesized as per $\mathbf{S 8}$ from 2-cyano- $N$-hexylacetamide $\mathbf{S 6}$ and 3-bromobenzaldehyde. Afforded a white solid, $19 \%$.

${ }^{1} \mathrm{H}$ NMR (DMSO): $8.13(1 \mathrm{H}, \mathrm{s}), 8.11(1 \mathrm{H}, \mathrm{s}), 7.93(1 \mathrm{H}, \mathrm{d}), 7.77(1 \mathrm{H}, \mathrm{d}), 7.53(1 \mathrm{H}, \mathrm{t}), 3.19(2 \mathrm{H}, \mathrm{t}), 1.47-1.49(2 \mathrm{H}$, m), 1.33-1.38 (2H, m), 1.25-1.28 (2H, m), $0.86(3 \mathrm{H}, \mathrm{t})$.

${ }^{13}$ C NMR (DMSO): 160.3, 148.6, 132.2, 134.5, 131.2, 128.6, 122.1, 115.9, 108.0, 39.4, 30.9, 28.7, 25.9, 21.9, 13.8.

2-cyano-N-hexyl-3-(4-chlorophenyl)acrylamide (S29). 
Synthesized as per S8 from 2-cyano- $N$-hexylacetamide $\mathbf{S 6}$ and 4-chlorobenzaldehyde. Afforded a white solid, $66 \%$.

${ }^{1} \mathrm{H}$ NMR (DMSO): 8.14 (1H, s), 7.94 (1H, d), 7.63 (1H, d), 3.19 (2H, t), 1.47-1.51 (2H, m), 1.33-1.38 (2H, m), 1.25-1.28 (2H, m), $0.85(3 \mathrm{H}, \mathrm{t})$.

${ }^{13}$ C NMR (DMSO): 160.5, 148.9, 136.7, 131.6, 130.7, 116.1, 107.0, 39.6, 30.9, 28.7, 25.9, 21.9, 13.8.

2-cyano-N-hexyl-3-(3-methoxyphenyl)acrylamide (S30).

Synthesized as per S8 from 2-cyano- $N$-hexylacetamide $\mathbf{S 6}$ and 3-methoxybenzaldehyde. Afforded a white solid, $30 \%$.

${ }^{1} \mathrm{H}$ NMR (DMSO): 8.12 (1H, s), 7.52 (1H, t), 7.50 (1H, d), 7.47 (1H, s), $7.16(1 \mathrm{H}, \mathrm{d}), 3.81(3 \mathrm{H}, \mathrm{s}), 3.20$ (2H, t), 1.47-1.51 (2H, m), 1.34-1.38 (2H, m), 1.25-1.28 (2H, m), $0.86(3 \mathrm{H}, \mathrm{t})$.

${ }^{13}$ C NMR (DMSO): 161.3, 156.9, 155.2, 133.0, 131.3, 118.7, 116.4, 113.4, 111.9, 105.8, 55.2, 39.4, 30.9, 28.7, 25.7, 21.9, 13.9 .

2-cyano-N-hexyl-3-(4-methoxyphenyl)acrylamide (S31).

Synthesized as per S8 from 2-cyano- $N$-hexylacetamide $\mathbf{S 6}$ and 4-methoxybenzaldehyde. Afforded a pale yellow solid, $65 \%$.

${ }^{1} \mathrm{H}$ NMR (DMSO): 8.08 (1H, s), 7.94-7.97 (1H, d), 7.10-7.13 (1H, d), 3.36 (3H, s), 3.18-3.20 (2H, t), 1.46-1.50 (2H, m), 1.34-1.38 (2H, m), 1.26-1.28 (2H, m), 0.83-0.88 (3H, t).

${ }^{13}$ C NMR (DMSO): 162.4, 161.1, 149.7, 132.3, 124.4, 116.9, 114.7, 102.7, 55.5, 39.4, 30.9, 28.8, 26.0, $21.9,13.8$.

2-cyano-N-hexyl-3-(3,4-dimethoxyphenyl)acrylamide (S32).

Synthesized as per S8 from 2-cyano- $N$-hexylacetamide $\mathbf{S 6}$ and 3,4-dimethoxybenzaldehyde. Afforded a white solid, $45 \%$.

${ }^{1} \mathrm{H}$ NMR (DMSO): 8.07 (1H, s), 7.56 (1H, d), 7.13(1H, d), 6.75 (1H, s), 3.85 (3H, s), 3.80 (3H, s), 3.19 (2H, t), 1.46-1.50 (2H, m), 1.33-1.38 (2H, m), 1.26-1.28 (2H, m), 0.85 (3H, t).

${ }^{13} \mathrm{C}$ NMR (DMSO): 161.0, 152.3, 150.2, 148.6, 125.3, 117.1, 112.1, 111.6, 102.6, 64.4, 55.6, 55.3, 39.6, 30.9, 28.8, 26.0, 22.0, 13.8 .

2-cyano-N-hexyl-3-(3-hydroxy-4-methoxyphenyl)acrylamide (S33).

Synthesized as per S8 from 2-cyano- $N$-hexylacetamide $\mathbf{S 6}$ and 3-hydroxy-4-methoxybenzaldehyde. Afforded a yellow solid, 97\%.

${ }^{1}$ H NMR (DMSO): 7.97 (1H, s), 7.39 (1H, d), 7.26 (1H, s), 7.07 (1H, d), 3.86 (3H, s), 3.19 (2H, t), 1.46-1.50 (2H, m), 1.33-1.38 (2H, m), 1.25-1.29 (2H, m), 0.85 (3H, t).

${ }^{13}$ C NMR (DMSO): 161.2, 1533, 150.0, 146.9, 129.7, 124.5, 116.9, 115.4, 113.4, 111.5, 55.7, 39.4, 30.9, 28.8, 26.0, 21.9, 13.8 .

2-cyano-N-hexyl-3-(3-nitrophenyl)acrylamide (S34).

Synthesized as per S8 from 2-cyano- $N$-hexylacetamide $\mathbf{S 6}$ and 3-nitrobenzaldehyde. Afforded a pale yellow solid, 33\%.

${ }^{1}$ H NMR (DMSO): 8.38 (1H, d), 8.34 (1H, s), 8.30 (1H, s), $8.31(1 \mathrm{H}, \mathrm{d}), 7.85(1 \mathrm{H}, \mathrm{t}), 3.19$ (2H, t), 1.47-1.50 (2H, $\mathrm{m}), 1.34-1.38(2 \mathrm{H}, \mathrm{m}), 1.25-1.28(2 \mathrm{H}, \mathrm{m}), 0.86(3 \mathrm{H}, \mathrm{t})$.

${ }^{13} \mathrm{C}$ NMR (DMSO): 160.1, 148.0, 147.5, 135.8, 133.4, 130.7, 126.1, 124.0, 115.7, 109.2, 39.1, 30.9, 28.6, 25.9, 21.9, 13.8 .

2-cyano-N-hexyl-3-(4-nitrophenyl)acrylamide (S35).

Synthesized as per S8 from 2-cyano- $N$-hexylacetamide $\mathbf{S 6}$ and 4-nitrobenzaldehyde. Afforded a white solid, $46 \%$.

${ }^{1} \mathrm{H}$ NMR (DMSO): $8.17(1 \mathrm{H}, \mathrm{s}), 7.01(1 \mathrm{H}, \mathrm{d}), 7.90(\mathrm{H}, \mathrm{d}), 3.19(2 \mathrm{H}, \mathrm{t}), 1.46-1.50(2 \mathrm{H}, \mathrm{m}), 1.33-1.38$ (2H, m), 1.25-1.28 (2H, m), 0.86 (3H, t).

${ }^{13}$ C NMR (DMSO): 161.7, 148.6, 147.3, 138.0, 130.0, 123.2, 116.3, 110.3, 39.4, 30.8, 28.6, 25.5, 21.8, 13.8. 
2-cyano-N-hexyl-3-(4-carboxyphenyl)acrylamide (S36).

Synthesized as per S8 from 2-cyano- $N$-hexylacetamide $\mathbf{S 6}$ and 4-carboxybenzaldehyde. Afforded a white solid, $83 \%$.

${ }^{1} \mathrm{H}$ NMR (DMSO): 8.20 (1H, s), 8.07 (1H, d), 7.99 (1H, d), 3.19 (2H, t), 1.47 (2H, m), 1.34-1.39 (2H, m), 1.26$1.29(2 \mathrm{H}, \mathrm{m}), 0.86(3 \mathrm{H}, \mathrm{t})$.

${ }^{13}$ C NMR (DMSO): 160.4, 149.0, 138.7, 135.7, 133.4, 129.8, 115.9, 108.5, 39.7, 30.9, 28.6, 25.9, 21.9, 13.8.

2-cyano-N-hexyl-3-(4-dimethylaminophenyl)acrylamide (S37).

Synthesized as per S8 from 2-cyano- $N$-hexylacetamide $\mathbf{S 6}$ and 4-(dimethylamino)benzaldehyde. Afforded a bright yellow solid, $81 \%$.

${ }^{1} \mathrm{H}$ NMR (DMSO): 7.94 (1H, s), 7.84 (1H, d), 6.79 (1H, d), 3.35 (3H, s), 3.04-3.18 (2H, t), 1.46-1.49 (2H, m), 1.33-1.38 (2H, m), 1.26-1.29 (2H, m), $0.86(3 \mathrm{H}, \mathrm{t})$.

${ }^{13}$ C NMR (DMSO): 161.9, 152.8, 150.0, 132.5, 124.4, 118.7, 111.5, 111.0, 39.5, 39.4, 30.9, 28.9, 26.0, 21.9, 13.8.

2-Cyano-N-(4-methoxybenzyl)acetamide (S7)

Synthesised as per S5 from methylcyanoacetate 4 (3 g, $30 \mathrm{mmol})$ and 4-methoxybenzylamine S3. Afforded a white solid, 68\%.

${ }^{1} \mathrm{H}$ NMR (DMSO): 8.72 (1H, s br), 7.3 (2H, d), 7.0 (2H, d), 4.32 (2H, s), 3.84 (3H, s), 3.76 (2H, s)

${ }^{13}$ C NMR (DMSO): 161.9, 158.3, 130.4, 128.7, 116.1, 113.7, 54.9, 42.1, 25.2

2-cyano-3-phenyl-N-(4-methoxybenzyl)acrylamide (S38).

Synthesised as per S8 from 2-cyano- $N$-(4-methoxybenzyl)acetamide S7 and benzaldehyde. Afforded (0.10 g, a white solid, $11 \%$.

${ }^{1} \mathrm{H}$ NMR (DMSO): 8.09 (1H, s), 7.39 (1H, d), $7.24(1 \mathrm{H}, \mathrm{t}), 7.18(1 \mathrm{H}, \mathrm{t}), 6.92(1 \mathrm{H}, \mathrm{d}), 6.84(1 \mathrm{H}, \mathrm{d}), 4.37$ (2H, s), $3.72(3 \mathrm{H}, \mathrm{s})$.

${ }^{13}$ C NMR (DMSO): 161.8, 158.6, 153.3, 142.4, 136.7, 131.0, 130.0, 129.4, 126.0, 116.3, 113.9, 101.8, 54.5, 42.6.

2-cyano-3-(3-hydroxyphenyl)- $N$-(4-methoxybenzyl)acrylamide (S39).

Synthesised as per S8 from 2-cyano- $N$-(4-methoxybenzyl)acetamide $\mathbf{S 7}$ and 3-hydroxybenzaldehyde. Afforded an off-white solid, 35\%.

${ }^{1}$ H NMR (DMSO): 8.08 (1H, s), 7.35 (1H, t), 7.25 (1H, d), 6.98 (1H, d), 6.97 (1H, s), 6.88 (1H, d), 4.33 (2H, s), $3.72(3 \mathrm{H}, \mathrm{s})$.

${ }^{13}$ C NMR (DMSO): 160.9, 158.2, 157.7, 150.6, 133.0, 130.7, 130.2, 128.8, 121.4, 119.6, 116.2, 115.7, 113.6, 105.8, 54.9, 42.5 .

2-cyano-3-(4-hydroxyphenyl)- $\mathrm{N}$-(4-methoxybenzyl)acrylamide (S40).

Synthesised as per S8 from 2-cyano- $N$-(4-methoxybenzyl)acetamide $\mathbf{S 7}$ and 4-hydroxybenzaldehyde. Afforded an off-white solid, $39 \%$.

${ }^{1} \mathrm{H}$ NMR (DMSO): 8.07 (1H, d), 7.87 (1H, d), 7.24 (1H, d), 6.92 (1H, d), 6.88 (1H, d), 4.33 (2H, s), 3.73 (3H, s).

${ }^{13}$ C NMR (DMSO): 161.8, 161.4, 158.2, 150.4, 132.8, 131.0, 128.8, 122.8, 117.1, 116.1, 113.6, 100.9, 54.9, 42.5.

2-cyano-3-(3,4-dihydroxyphenyl)-N-(4-methoxybenzyl)acrylamide (S41).

Synthesised as per S8 from 2-cyano- $N$-(4-methoxybenzyl)acetamide $\mathbf{S 7}$ and 3,4-dihydroxybenzaldehyde. Afforded a yellow solid, 36\%.

${ }^{1} \mathrm{H}$ NMR (DMSO): 7.97 (1H, s), 7.55 (1H, s), 7.28 (1H, d), 7.23 (1H, d), 7.20 (1H, d), 6.87 (1H, d), 4.32 (2H, s), $3.71(3 \mathrm{H}, \mathrm{s})$.

${ }^{13} \mathrm{C}$ NMR (DMSO): 161.6, 158.2, 151.1, 150.7, 145.5, 131.0, 128.7, 125.4, 123.0, 117.2, 117.2, 115.8, 113.6, 100.2, 54.9, 42.5 .

2-cyano-3-(2-chlorophenyl)- $N$-(4-methoxybenzyl)acrylamide (S42).

Synthesised as per S8 from 2-cyano- $N$-(4-methoxybenzyl)acetamide $\mathbf{S 7}$ and 2-chlorobenzaldehyde. Afforded a pale yellow solid, $40 \%$. 
${ }^{1} \mathrm{H}$ NMR (DMSO): $8.38(1 \mathrm{H}, \mathrm{s}), 7.80(1 \mathrm{H}, \mathrm{d}), 7.64(1 \mathrm{H}, \mathrm{d}), 7.54(1 \mathrm{H}, \mathrm{t}), 7.54(1 \mathrm{H}, \mathrm{t}), 7.26(\mathrm{H}, \mathrm{d}), 6.89(\mathrm{H}, \mathrm{d})$, $4.36(2 \mathrm{H}, \mathrm{s}), 3.72(3 \mathrm{H}, \mathrm{s})$.

${ }^{13}$ C NMR (DMSO): 159.9, 158.3, 147.0, 134.0, 133.2, 130.6, 130.3, 130., 129.6, 128.9, 127.7, 115.3, 113.6, 110.4, 55.0, 42.7.

2-cyano-3-(3-bromophenyl)- $N$-(4-methoxybenzyl)acrylamide (S43).

Synthesised as per S8 from 2-cyano- $N$-(4-methoxybenzyl)acetamide S7 and 3-bromobenzaldehyde. Afforded an off-white solid, $46 \%$.

${ }^{1} \mathrm{H}$ NMR (DMSO): 8.10 (1H, s), 7.80 (1H, s), 7.75 (1H, d), 7.57 (1H, d), $7.23(1 \mathrm{H}, \mathrm{t}), 7.18$ (1H, d), $6.88(1 \mathrm{H}, \mathrm{d})$, 4.34 (2H, s), 3.71 (3H, s).

${ }^{13}$ C NMR (DMSO): 162.1, 158.5, 154.9, 135.7, 133.8, 131.3, 129.5, 128.5, 128.3, 124.8, 123.1, 116.8, 113.4, 101.7, 54.5, 42.6

2-cyano-3-(4-chlorophenyl)- $N$-(4-methoxybenzyl)acrylamide (S44).

Synthesised as per S8 from 2-cyano- $N$-(4-methoxybenzyl)acetamide S7 and 4-chlorobenzaldehyde. Afforded a pale yellow solid, $34 \%$.

${ }^{1} \mathrm{H}$ NMR (DMSO): 8.19 (1H, s), 7.42 (1H, d), 7.34 (1H, d), 7.23 (1H, d), 6.84 (1H, d), 4.18 (2H, s), 3.72 (3H, s).

${ }^{13}$ C NMR (DMSO): 162.80, 160.69, 158.11, 135.01, 133.33, 129.90, 129.40, 126.73, 119.58, 113.52, 100.01, 54.94, 43.01.

2-cyano-3-(3-methoxyphenyl)-N-(4-methoxybenzyl)acrylamide (S45).

Synthesised as per S8 from 2-cyano- $N$-(4-methoxybenzyl)acetamide $\mathbf{S 7}$ and 3-methoxybenzaldehyde. Afforded an off-white solid24\%.

${ }^{1} \mathrm{H}$ NMR (DMSO): 8.18 (1H, s), $7.51(1 \mathrm{H}, \mathrm{t}), 7.48(1 \mathrm{H}, \mathrm{s}), 7.26(1 \mathrm{H}, \mathrm{d}), 7.16(1 \mathrm{H}, \mathrm{d}), 6.89(1 \mathrm{H}, \mathrm{d}), 4.36(2 \mathrm{H}, \mathrm{s})$, $3.75(3 \mathrm{H}, \mathrm{s})$.

${ }^{13}$ C NMR (DMSO): 161.78, 160.73, 159.31, 158.24, 133.07, 130.69, 130.25, 128.81, 118.12, 116.27, 114.77, $113.60,111.21,106.42,54.95,42.56$.

2-cyano-3-(4-methoxyphenyl)-N-(4-methoxybenzyl)acrylamide (S46).

Synthesised as per S8 from 2-cyano- $N$-(4-methoxybenzyl)acetamide $\mathbf{S 7}$ and 4-methoxybenzaldehyde. Afforded a pale yellow solid, $42 \%$.

${ }^{1} \mathrm{H}$ NMR (DMSO): 8.12 (1H, s), 7.95 (1H, d), 7.23 (1H, d), 7.11 (1H, d), 6.87 (1H, d), 4.32 (2H, s), 3.71 (3H, s).

${ }^{13}$ C NMR (DMSO): 162.50, 161.18, 158.20, 150.13, 132.40, 130.88, 128.79, 128.77, 116.90, 114.74, 113.58, 102.43, 54.94, 42.51 .

2-cyano-3-(3,4-dimethoxyphenyl)-N-(4-methoxybenzyl)acrylamide (S47).

Synthesised as per S8 from 2-cyano- $N$-(4-methoxybenzyl)acetamide $\mathbf{S 7}$ and 3,4-dimethoxybenzaldehyde. Afforded an off-white solid, 58\%.

${ }^{1}$ H NMR (DMSO): 8.11 (1H, s), 7.57 (1H, d), 7.23 (1H, d), 7.13 (1H, d), 6.88 (1H, d), 6.59 (1H, s), 4.32 (12H, s), $3.34(13 \mathrm{H}, \mathrm{s})$.

${ }^{13} \mathrm{C}$ NMR (DMSO): 161.18, 158.20, 152.41, 150.54, 148.55, 130.89, 128.78, 128.75, 117.03, 113.58, 112.22 , 111.72, 102.38, 98.78, 55.69, 54.95, 42.51.

2-cyano-3-(3-hydroxy-4-methoxyphenyl)-N-(4-methoxybenzyl)acrylamide (S48).

Synthesised as per S8 from 2-cyano- $N$-(4-methoxybenzyl)acetamide S7 and 3-hydroxy-4-methoxybenzaldehyde. Afforded a yellow solid, $45 \%$.

${ }^{1} \mathrm{H}$ NMR (DMSO): 8.02 (1H, s), 7.54 (1H, s), 7.40 (1H, d), 7.24 (1H, d), 7.09 (1H, d), 6.89 (1H, d), 4.32 (2H, s), $3.86(3 \mathrm{H}, \mathrm{s}), 3.73(3 \mathrm{H}, \mathrm{s})$.

${ }^{13} \mathrm{C}$ NMR (DMSO): 161.33, 158.19, 151.71, 150.42, 146.63, 130.91, 128.75, 124.62, 119.26, 116.85, 115.40, 113.58, 111.95, 101.99, 55.64, 54.95, 42.49.

2-cyano-3-(3-nitrophenyl)-N-(4-methoxybenzyl)acrylamide (S49). 
Synthesised as per S8 from 2-cyano- $N$-(4-methoxybenzyl)acetamide S7 and 3-nitrobenzaldehyde. Afforded a yellow solid, 69\%.

${ }^{1} \mathrm{H}$ NMR (DMSO): 8.41 (1H, s), 8.19 (1H, s), 7.93 (1H, d), $7.81(1 \mathrm{H}, \mathrm{d}), 7.57(1 \mathrm{H}, \mathrm{t}), 7.17$ (1H, d), $6.86(1 \mathrm{H}, \mathrm{d})$, $4.36(2 \mathrm{H}, \mathrm{s}), 3.71(3 \mathrm{H}, \mathrm{s})$.

${ }^{13}$ C NMR (DMSO): 161.27, 158.62, 153.61, 145.71, 135.57, 135.38, 130.57, 130.02, 128.36, 122.59, 121.39, 116.12, 113.37, 101.62, 54.67, 42.73.

2-cyano-3-(4-nitrophenyl)-N-(4-methoxybenzyl)acrylamide (S50).

Synthesised as per S8 from 2-cyano- $N$-(4-methoxybenzyl)acetamide S7 and 4-nitrobenzaldehyde. Afforded a white solid, $51 \%$.

${ }^{1} \mathrm{H}$ NMR (DMSO): 8.27 (1H, s), 7.80 (1H, d), 7.54 (1H, d), 6.94 (1H, d), 6.85 (1H, d), 4.35 (2H, s), 3.75 (3H, s).

${ }^{13} \mathrm{C}$ NMR (DMSO): 161.87, 158.94, 155.17, 148.36, 139.69, 133.79, 129.89, 129.15, 122.63, 116.84, 113.62, 101.72, 54.61, 42.84 .

2-cyano-3-(4-carboxyphenyl)- N-(4-methoxybenzyl)acrylamide (S51).

Synthesised as per S8 from 2-cyano- $N$-(4-methoxybenzyl)acetamide S7 and 4-carboxybenzaldehyde. Afforded an off-white solid, $22 \%$.

${ }^{1} \mathrm{H}$ NMR (DMSO): 8.26 (1H, s), 8.09 (1H, d), 7.80 (1H, d), 7.26 (1H, d), 6.90 (1H, d), 4.35 (2H, s), 3.73 (3H, s).

${ }^{13}$ C NMR (DMSO): 168.93, 166.45, 160.50, 158.26, 149.43, 135.63, 133.56, 130.57, 129.91, 128.83, 115.89, 113.62, 108.30, 54.95, 42.18.

2-cyano-3-(4-dimethylaminophenyl)-N-(4-methoxybenzyl)acrylamide (S52).

Synthesised as per S8 from 2-cyano- $N$-(4-methoxybenzyl)acetamide S7 and 4-(dimethylamino)benzaldehyde. Afforded a bright yellow solid, 55\%.

${ }^{1} \mathrm{H}$ NMR (DMSO): 8.00 (1H, s), 7.85 (1H, d), 7.24 (1H, d), 6.88 (1H, d), 6.79 (1H, d), 4.34 (2H, s), 3.72 (3H, s), $3.06(3 \mathrm{H}, \mathrm{s})$.

${ }^{13} \mathrm{C}$ NMR (DMSO): 161.99, 158.13, 152.81, 150.39, 132.59, 128.74, 128.69, 123.96, 118.64, 113.54, 111.50 , 96.66, 54.94, 47.92, 42.43. 


\section{Figure S2\#1 (Over). Degradation of 1 produces multiple products determined by HPLC and mass}

spectrometry.

Two $10 \mathrm{ml}$ lots of $200 \mu \mathrm{M} 1$ were prepared. A control was frozen and the other was left to oxidise under air at room temperature $\left(22{ }^{\circ} \mathrm{C}\right)$ for 16 hours. A $50 \mu$ l aliquot of each was analysed with HPLC using a gradient from $100 \%$ phase A ( $0.1 \%$ TFA in water) to $25 \%$ phase B $(0.1 \%$ TFA in acetonitrile) in 1.5 minutes and then to $40 \%$ phase $\mathrm{B}$ in 7.5 minutes. The chromatograms for:

(a) the control sample; and

(b) the 16 hr 1 are shown.

The peak at 5.63 minutes for $\mathbf{1}$ in the control sample is diminished in the $16 \mathrm{hr}$ sample and there are a number of new peaks. Two major degradation products eluted earlier than $\mathbf{1}$ at 4.29 and 4.59 minutes and one later at 6.91 minutes. The remainder of each $10 \mathrm{ml}$ solution was concentrated on a C8 $1 \mathrm{ml}$ SPE cartridge (Amersham) to $250 \mu \mathrm{l}$ and the volume was reduced further in a vacuum centrifuge to a saturated solution ( 20 $\mu \mathrm{l})$. A $0.5 \mu \mathrm{l}$ aliquot of each was mixed with $0.5 \mu \mathrm{l}$ of $0.2 \%$ formic acid in acetonitrile and were sprayed in a nanoelectrospray tip (EconoTip, Proxeon) at $1200 \mathrm{~V}$ using a quadrupole-time-of-flight mass spectrometer (QSTAR XL, Applied Biosystems).

The mass spectra for: (c) the control 1; and

(d) the $16 \mathrm{hr} \mathbf{1}$ are shown. In the control sample, $\mathbf{1}$ at m/z 221.03 (monoisotopic mass for the [M+H] $]^{+}$ion) is the major signal. Whereas, in the $16 \mathrm{hr}$ sample the A47 is reduced and the spectrum is now dominated by two signals at $\mathrm{m} / \mathrm{z} 219.02$ and 308.98. Several new signals are present at m/z 366.95, 408.99, 444.97, 574.94 and 652.96. These signals in (d) indicate than the degradation products observed chromatographically in (b) have recombined to produce larger molecules than 1, which may have effects on dynamin GTPase activity distinct from 1.

See Supplementary data \#2 for figure 


\section{REFERENCES}

1. Gazit, A.; Osherov, N.; Gilon, C.; Levitzki, A. Tyrphostins. 6. Dimeric Benzylidenemalononitrile Tyrphostins: Potent Inhibitors of EGF Receptor Tyrosine Kinase in Vitro. J. Med. Chem. 1996, 39, 4905-4911.

2. Thompson, M.; Rewcastle, G. W.; Tercel, M.; Dobrusin, E. M.; Fry, D. W.; Kraker A. J.; Denny, W. A. Tyrosine Kinase Inhibitors. 1. Structure-Activity Relationships for Inhibition of Epidermal Growth Factor Receptor Tyrosine Kinase Activity by 2,3-Dihydro-2-thioxo-1H-indole-3-a1kanoic Acids and 2,2'-Dithiobis(lH-indole-3-alkanoic acids). J. Med. Chem. 1993, 36, 2459-2469.

3. Namazi, H.; Assadpour, A.; Pourabbas, B.; Entezami, A. Polycondensation of bis(cyanoacetate) and 5a,10b-dihydrobenzofuro[2,3-b]benzofuran-2,9-dicarbaldehyde via Knoevenagel reaction: synthesis of donor-acceptor polymers containing shoulder-to-shoulder main chains. J. App. Poly. Sci. 2001, 81, 505-511.

4. Benoist, E.; Loussouarn, A.; Remaud, P.; Chatal, J.-F.; Gestin, J.-F. Convenient and simplified approaches to $\mathrm{N}$-monoprotected triaminopropane derivatives: Key intermediates for bifunctional chelating agent synthesis. Synthesis, 1998, 113-118. 